\title{
Investigation of the Immunomodulatory Properties of a Novel Allergen Variant That Targets Human Dendritic Cells
}

\author{
Kathryn Elizabeth Hulse \\ Waukesha, Wisconsin
}

Bachelor of Science, University of Wisconsin-Eau Claire, 2000

A Dissertation presented to the Graduate Faculty of the University of Virginia in Candidacy for the Degree of Doctor of Philosophy

Department of Microbiology

University of Virginia

December, 2008

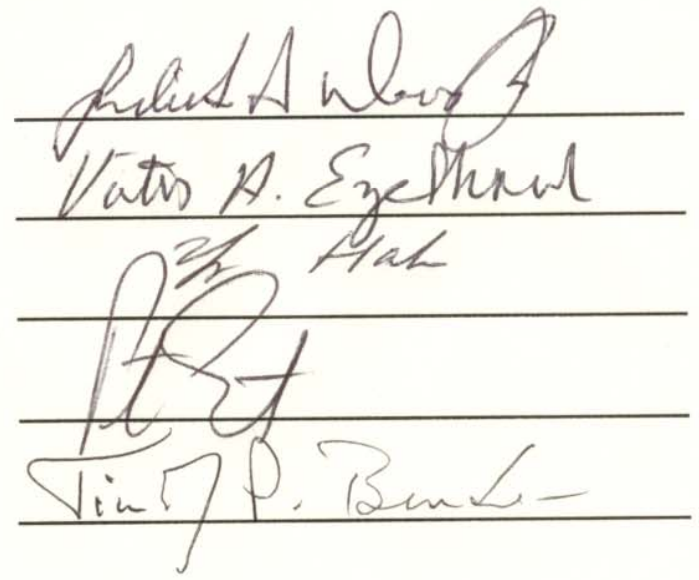




\section{Abstract}

The allergen variant $\mathrm{H} 22-\mathrm{Fel} \mathrm{d} 1$ targets cat allergen to the high affinity IgG receptor FcyRI on DCs. This molecule was developed as a possible treatment for cat-allergic patients. The objective of this dissertation was to investigate the immunomodulatory properties of $\mathrm{H} 22-\mathrm{Fel} \mathrm{d} 1$ using an in vitro human-based system. H22-Fel d 1 induced a semi-mature phenotype in monocyte-derived dendritic cells (moDCs), characterized by enhanced production of Th1-associated cytokines with no change in expression of costimulatory molecules. Furthermore, H22-Fel d 1-primed moDCs induced a selective increase in Th2 and IL-10-expressing regulatory T cell types pointing to qualitative changes in the T cell cytokine repertoire. Importantly, this effect was restricted to cat-allergic subjects. Blockade of IL-10 selectively enhanced the Th2 component of the $\mathrm{T}$ cell response to $\mathrm{H} 22-\mathrm{Fel} \mathrm{d} 1$, suggesting that this arm of the response was regulated by IL-10. The clinical efficacy of $\mathrm{H} 22-\mathrm{Fel} \mathrm{d} 1$ will likely depend on its capacity to induce a protective $\mathrm{T}$ cell cytokine repertoire in a "pro-allergic" cytokine milieu in vivo. Therefore, we investigated whether the Th2promoting cytokine, thymic stromal lymphopoietin (TSLP), which acts directly on

DCs and is expressed in the skin and lungs of patients with allergic disease, mitigates the immunomodulatory capacity of H22-Fel d 1. Surprisingly, TSLP was a weak inducer of Th2 responses in vitro when, irrespective of atopic status; however, when moDCs were co-primed with TSLP and H22-Fel d 1 Th2 responses were amplified in a synergistic manner in highly atopic subjects. This effect was OX40 ligand-independent and not associated with secretion of the 
Th2-attracting chemokine, CCL17, pointing to an unconventional TSLPmediated pathway. Expression of TSLP receptor was markedly enhanced on atopic moDCs after priming with $\mathrm{H} 22-\mathrm{Fel} \mathrm{d} 1$ through a pathway regulated by PI3K and PKC. Moreover, inhibition of these molecules selectively abolished TSLP-mediated Th2 responses triggered by H22-Fel d 1. Discovery of a novel pathway linking Fc $\gamma \mathrm{RI}$ to TSLP receptor upregulation and consequent TSLPmediated effects questions the validity of allergen vaccines which engage surface receptors on DCs, but serendipitously identifies DC signaling components which could serve as new therapeutic targets for Th2-driven diseases. 


\section{Acknowledgments}

There are several people whose guidance and support were critical to my success during my doctoral studies. First and foremost, I would like to thank my mentor Dr. Judith Woodfolk for allowing me to complete my graduate work under her mentorship. Her guidance has been invaluable to me both scientifically and personally, and I know she has prepared me for the years ahead in academic research. I would also like to thank my co-mentor Dr. Victor Engelhard, as well as the rest of my thesis committee Dr. Timothy Bender, Dr. Young Hahn, Dr. Peter Ernst and Dr. Klaus Ley for their advice and guidance.

I am also grateful to the members of the Allergy Division, especially Dr. Thomas Platts-Mills for many insightful discussions. I would also like to thank Amanda Urban not only for all of the technical assistance, but also for her invaluable friendship over the years. I am indebted to Holly Carper and Deb Murphy for all of their help with blood draws. I also thank Dr. Martin Chapman and Lisa Vailes for providing me with valuable reagents. I would like to acknowledge the many members of the Carter Immunology Center who have assisted me along the way, as well as James Patrie for his assistance with the statistical analysis of $\mathrm{T}$ cell data.

Finally, I cannot thank my husband David enough for his enduring love, support and encouragement of my goals. My family has also provided much needed love and support. 


\section{List of Abbreviations}

$\begin{array}{ll}\text { Ab } & \text { Antibody } \\ \text { AHR } & \text { Airway hyperresponsivness } \\ \text { ANOVA } & \text { Analysis of variance } \\ \text { APC } & \text { Allophycocyanin } \\ \text { APC } & \text { Antigen presenting cell } \\ \text { aTreg } & \text { Adaptive regulatory T cell } \\ \text { Bet v } & \text { Betulla verrucosa } \\ \text { CD } & \text { Cluster of differentiation } \\ \text { CDR } & \text { Complementarity-determining region } \\ \text { ChIP } & \text { Chromatin immunoprecipitation } \\ \text { CI } & \text { Confidence interval } \\ \text { CLA } & \text { Cutaneous lymphocyte-associated antigen } \\ \text { CRTH2 } & \text { Chemoattractant receptor-homologous molecule expressed on Th2 } \\ & \text { cells } \\ \text { DAG } & \text { Diacylglycerol } \\ \text { DC } & \text { Dendritic cell } \\ \text { Der p } & \text { Dermatophagoides pteronyssinus } \\ \text { ELISA } & \text { Enzyme-linked immunosorbent assay } \\ \text { EMSA } & \text { Elecrticmobility shift assay } \\ \text { EU } & \text { Endotoxin unit } \\ \text { FACS } & \text { Fluorescence-activated cell sorting } \\ \text { FBS } & \text { Fetal bovine serum } \\ \text { FcR } & \text { Fc receptor } \\ \text { Fel d } & \text { Felis domesticus } \\ \text { FGF } & \text { Fibroblast growth factor } \\ \text { FITC } & \text { Fluorescein isothiocyanate } \\ \text { FSC } & \text { Forward scatter } \\ \text { g } & \text { Gram } \\ \gamma_{c} & \text { IL-2 receptor common gamma chain } \\ \text { GM-CSF } & \text { Granulocyte macrophage colony-stimulating factor } \\ \text { HLA } & \text { Human leukocyte antigen } \\ \text { IFN } & \text { Interferon } \\ \text { Ig } & \text { Immunoglobulin } \\ \text { IDEC } & \text { Inflammatory dendritic epithelial cells } \\ \text { IL } & \text { Interleukin } \\ \text { IL-7r } & \text { Interleukin 7 receptor } \\ \text { IP-10 } & \text { Interferon-inducible protein 10 } \\ \text { ITAM } & \text { Immunoreceptor tyrosine-based activation motif } \\ \text { ITIM } & \text { Immunoreceptor tyrosine-based inhibition motif } \\ \text { IU } & \text { International unit } \\ \text { Jak } & \text { Janus kinase } \\ \text { kDa } & \text { Kilodalton } \\ \text { LC } & \text { Langerhans cells } \\ & \\ & \end{array}$




\begin{tabular}{|c|c|}
\hline LPS & Lippopolysaccharide \\
\hline $\mathrm{mAb}$ & Monoclonal antibody \\
\hline MACS & Magnetic-activated cell sorting \\
\hline MCP & monocyte chemoattractant protein \\
\hline MDC & Macrophage-derived chemokine \\
\hline MHC & Major histocompatibility complex \\
\hline MIP & Macrophage inflammatory protein \\
\hline $\operatorname{moDC}$ & Monocyte-derived dendritic cell \\
\hline$\mu \mathrm{Ci}$ & Microcurie \\
\hline$\mu \mathrm{g}$ & Microgram \\
\hline $\mathrm{mg}$ & Milligram \\
\hline $\mathrm{ml}$ & Milliliter \\
\hline NF & Nuclear factor \\
\hline ng & Nanogram \\
\hline NS & No stimulus (unstimulated) \\
\hline nTreg & Natural regulatory T cell \\
\hline OVA & Ovalbumin \\
\hline OX40L & OX40 ligand \\
\hline PAGE & Polyacrylamide gel electrophoresis \\
\hline PAMP & Pathogen-associated molecular pattern \\
\hline PBMC & Perhipheral blood mononuclear cell \\
\hline PCR & Polymerase chain reaction \\
\hline PDGF & Platelet-derived growth factor \\
\hline PE & Phycoerythrin \\
\hline PerCP & Peridinin-chlorophyll-protein \\
\hline pg & Picogram \\
\hline PHA & Phytohemagglutinin \\
\hline PI3K & Phosphoinositol-3 kinase \\
\hline PIP & Phosphoinositol phosphate \\
\hline PKC & Protein kinase $\mathrm{C}$ \\
\hline PLC & Phospholipase C \\
\hline PMA & phorbol 12-myristate 13 -acetate \\
\hline PRR & Pattern recognition receptor \\
\hline RANTES & Regulated upon activation, normal T cell expressed and secreted \\
\hline RT-PCR & Reverse transcriptase PCR \\
\hline SD & Standard deviation \\
\hline SDS & Sodium dodecyl sulfate \\
\hline SEM & Standard error of the mean \\
\hline sFv & single-chain antibody fragment variable region \\
\hline $\mathrm{SH} 2$ & Src-homology 2 \\
\hline SiRNA & Small interfering ribonucleic acid \\
\hline SIT & Specific immunotherapy \\
\hline SOCS & Suppressor of cytokine signaling \\
\hline SRTK & Src-related tyrosine kinase \\
\hline SSC & Side scatter \\
\hline
\end{tabular}




$\begin{array}{ll}\text { Stat } & \text { Signal transducer and activator of transcription } \\ \text { TARC } & \text { Thymus and activation-related chemokine } \\ \text { Tcm } & \text { Central memory T cell } \\ \text { Tem } & \text { Effector memory T cell } \\ \text { TLR } & \text { Toll-like receptor } \\ \text { TGF } & \text { Transforming growth factor } \\ \text { TNF } & \text { Tumor necrosis factor } \\ \text { Tr1 } & \text { Type 1 regulatory T cell } \\ \text { Treg } & \text { Regulatory T cell } \\ \text { TSLP } & \text { Thymic stromal lymphopoietin } \\ \text { TSLPr } & \text { TSLP receptor } \\ \text { VEGF } & \text { Vascular endothelial growth factor } \\ \text { VH } & \text { Heavy chain variable region } \\ \text { VL } & \text { Light chain variable region }\end{array}$




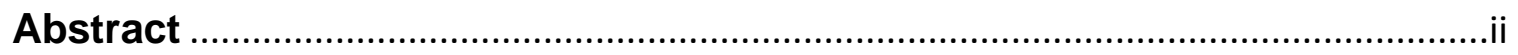

Acknowledgments ............................................................................................

Table of Contents .................................................................................................

List of Figures ....................................................................................................

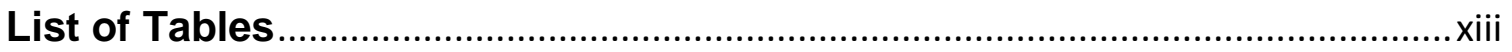

Chapter I - Introduction ...................................................................................... 1

Clinical Manifestations of Allergic Disease ........................................................ 1

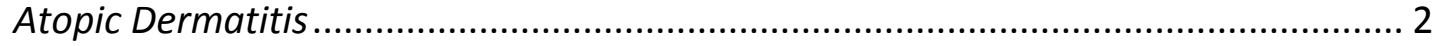

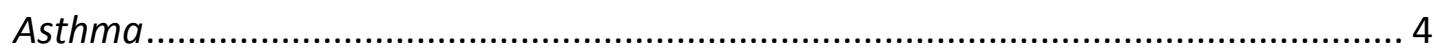

Allergic Rhinitis ............................................................................................... 5

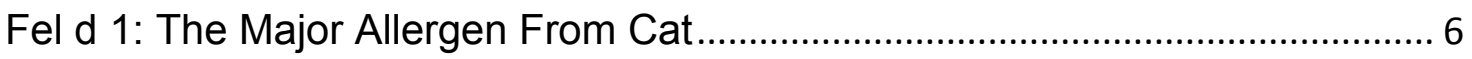

The Role of Th2 Cells in Allergic Inflammation................................................... 10

What Constitutes a Protective T Cell Response? .................................................. 12

Evidence of "Tolerance" to Inhaled Allergens ......................................................... 14

Immunomodulatory Effects of Immunotherapy ....................................................... 15

Current Approaches to T Cell Modulation ............................................................ 16

Features of a Novel Allergen Variant Targeted to Dendritic Cells ...................... 18

Targeting Antigens to FCYRI ................................................................................ 19

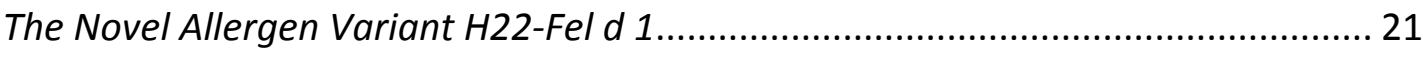

Thymic Stromal Lymphopoietin - A Potential Immunomodulator In Vivo? ....... 24

Goals of the Dissertation .................................................................................. 32

Chapter II - Targeting Fel d 1 to Fc $\gamma R$ I Induces a Novel Variation of the Th2 Response in Subjects with Cat Allergy ............................................................... 37

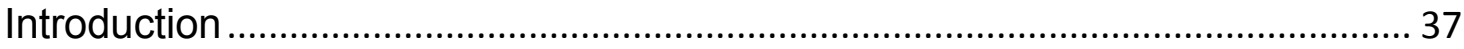

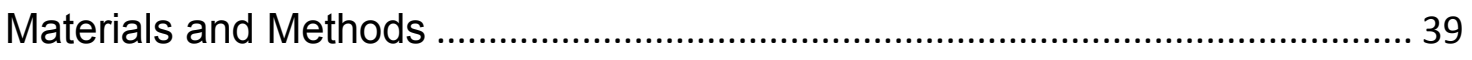

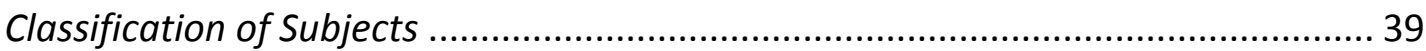

Induction of cytokines in monocyte-derived dendritic cells ....................................... 41

CD4 $4^{+}$-cell/moDC co-cultures........................................................................ 41 
ix

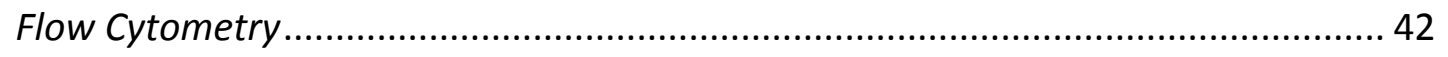

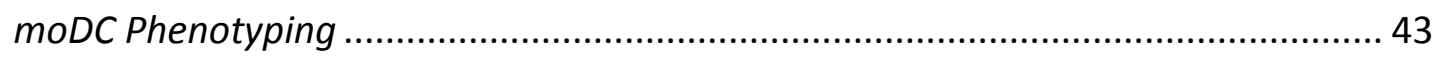

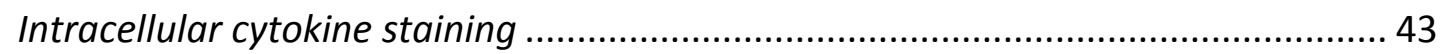

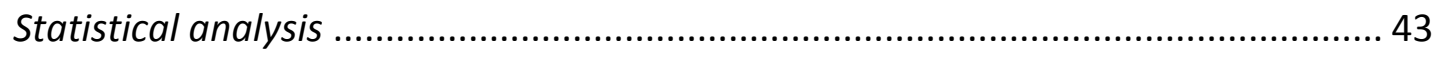

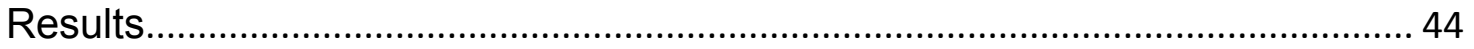

H22-Fel $d 1$ induces production of inflammatory cytokines by dendritic cells without increasing surface markers of maturation ........................................................... 44

H22-Fel d 1 induces increased proliferation in PBMC cultures ............................... 54

H22-Fel d 1 amplifies IL-5- and IL-10-expressing CD4 ${ }^{+} T$ cells and this effect is restricted to cat-allergic subjects ............................................................................. 54

H22-Fel $d 1$ induces cytokine-secreting subtypes consistent with ThO and regulatory

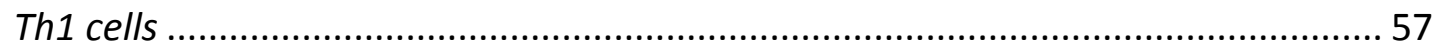

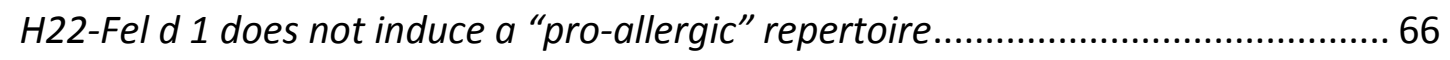

IL-10 induced by H22-Fel d 1 selectively controls Th2 cells ................................... 66

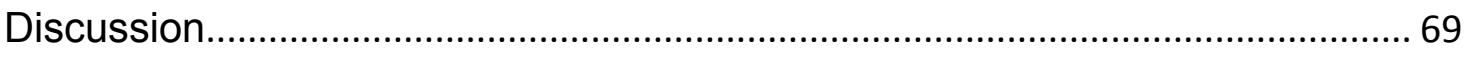

\section{Chapter III - Targeting Allergen to Fc $\gamma$ RI Reveals a Novel Pathway Which} Regulates Th2 Responses in Atopic Individuals.......................................... 76

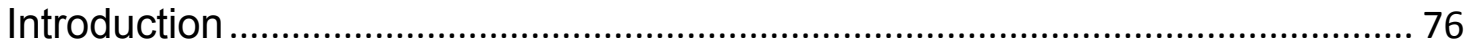

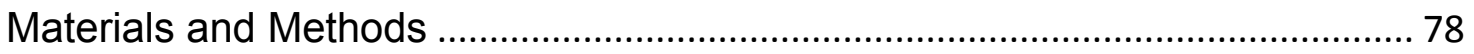

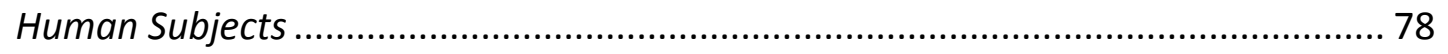

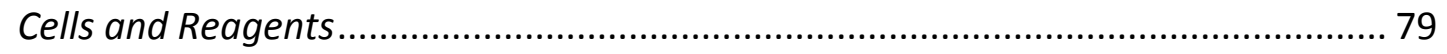

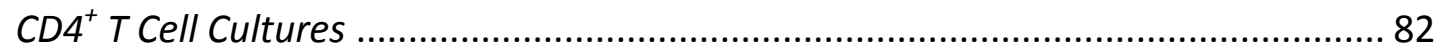

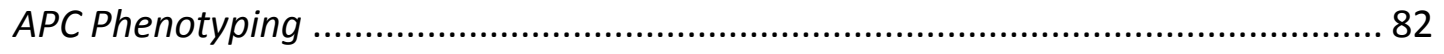

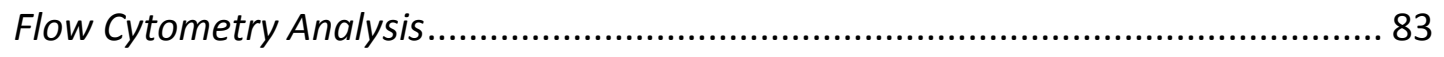

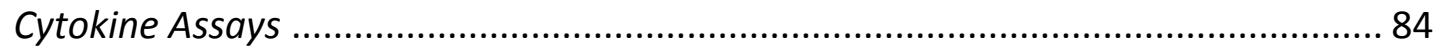

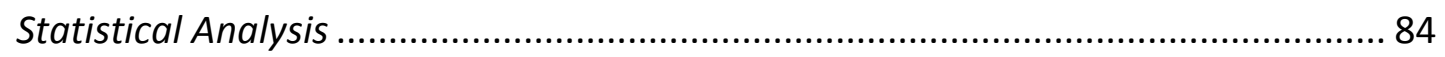

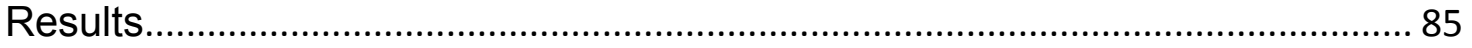

The Th2-Promoting Effects of TSLP are Increased in Atopic Subjects....................... 85

Dendritic Cells Primed with H22-Fel d 1 and TSLP Provide a Potent Th2 Stimulus in Atopic Subjects. 
The Effects of TSLP are APC-Mediated $\quad x$

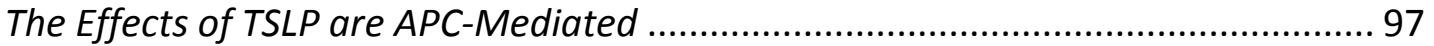

The Th2-Promoting Effect of TSLP on H22-Fel d 1-primed moDCs is OX4O Ligand-

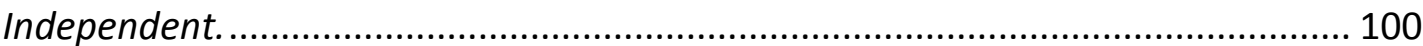

H22-Fel d 1 Enhances TSLP Receptor Expression in Atopic MoDCs Through a Pathway Which is Regulated by PISK and PKC. ................................................................ 107

TSLP Receptor is Weakly Induced by Low Dose LPS............................................ 115 Inhibition of PI3K and PKC in Atopic MoDCs Abolishes the Th2-Promoting Effect of

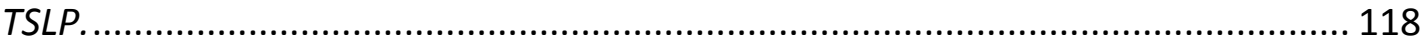

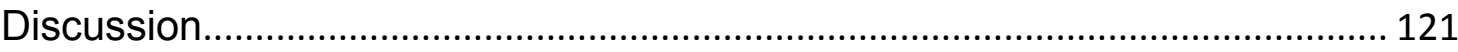

Chapter IV- Conclusions and Future Directions......................................... 129

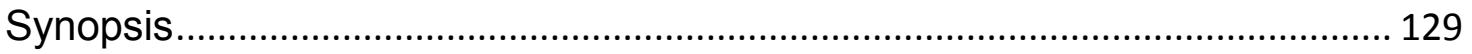

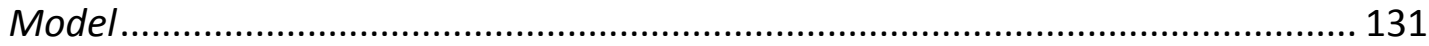

Future Directions ....................................................................................... 134

Examine accessibility of the ts/pr gene locus in moDCs from atopic and non-atopic

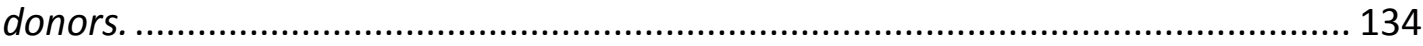

Investigation of the transcription factors involved in ts/pr gene expression........... 138 Investigate the role of PI3K and PKC in activation of transcription factors required for tslpr expression. ......................................................................................... 140

Final Conclusions and Clinical Implications ................................................. 141

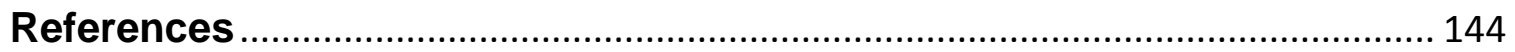




\section{List of Figures}

Figure 1.1 Three-Dimensional Structure of Fel d 1 Indicating the Location of IL10- and IFN- $\gamma$-Inducing Epitopes. 7

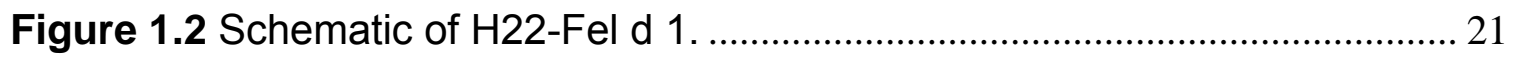

Figure 1.3 Schematic of the High Affinity TSLP Receptor....................................... 26

Figure 1.4 Effects of TSLP on Human Dendritic Cells.......................................... 29

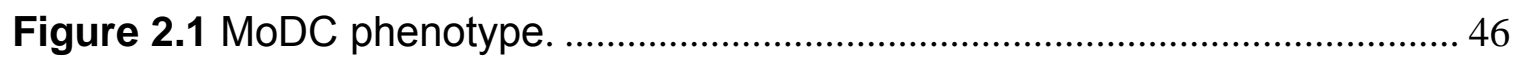

Figure 2.2 CD64 is Expressed on MoDCs.......................................................... 48

Figure 2.3 H22-Fel d 1 Does Not Alter Expression of Co-Stimulatory Molecules.

Figure 2.4 H22-Fel d 1 Enhances Production of Th1-Associated Cytokines and IL-10. 52

Figure 2.5 Proliferation of PBMCs is Significantly Increased by........................... 55

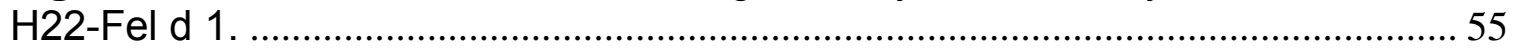

Figure 2.6 H22-Fel d 1 Enhances the Frequency of IL-5+ and IL-10+ T Cells. .. 58

Figure 2.7 Changes in Cytokine-Positive T cells Induced by H22-Fel d 1......... 60

Figure 2.8 H22-Fel d 1 Induces a Qualitative Change in the T Cell Repertoire.. 62

Figure 2.9 Cytokine-Positive T Cell Subtypes Induced by H22-Fel d 1. 64

Figure 2.10 The T Cell Repertoire Induced by H22-Fel d 1 is Distinct from a "ProAllergic" Repertoire.

Figure 2.11 IL-10 Blockade Selectively Enhances Th2 Responses. ..................... 70

Figure 3.1 The Th2-Promoting Effects of TSLP are Increased in Atopic Subjects. 86

Figure 3.2 Secretion of Th2-Promoting Cytokines is Increased in Atopic Subjects in Response to TSLP. 88

Figure 3.3 Dendritic Cells Primed with H22-Fel d 1 and TSLP Provide a Potent Th2 Stimulus in Atopic Subjects. 
Figure 3.4 Dendritic Cells Primed with H22-Fel d 1 and TSLP Provide a Potent Th2 Stimulus in Atopic Subjects.

Figure 3.5 T Cells Co-Cultured with Dendritic Cells Primed with H22-Fel d 1 and TSLP Secrete Increased Levels of Th2, but not Th1 Cytokines 95

Figure 3.6 TSLP Receptor is Expressed on a Small Percentage of CD4 ${ }^{+} \mathrm{T}$ Cells.

Figure 3.7 The Th2-Promoting Effects of TSLP on H22-Fel d 1-primed moDCs are OX40 Ligand-Independent.

Figure 3.8 OX40L Blockade Does Not Inhibit Induction of IL-4+ T Cells.

Figure 3.9 TSLP Has No Effect on Co-Stimulatory Molecule Expression or Cytokine Secretion Induced by H22-Fel d 1.

Figure 3.10 TSLP Receptor is Constitutively Expressed on Atopic MoDCs and Upregulated by $\mathrm{H} 22-\mathrm{Fel} \mathrm{d} 1$. 108

Figure 3.11 H22-Fel d 1-Induced TSLPr Expression Peaks at 24 Hours. 110

Figure 3.12 Serum from Atopic or Non-Atopic Subjects Does Not Affect TSLPr Expression.

Figure 3.13 H22-Fel d 1 Induces TSLP Receptor in Atopic moDCs Through a Pathway Which is Regulated by PI3K and PKC.

Figure 3.14 TSLP Receptor is Weakly Induced by Low Dose LPS.

Figure 3.15 Inhibition of PI3K and PKC Abolishes the Th2-Promoting Effect of TSLP.

Figure 4.1 Model of H22-Fel d 1-Induced TSLPr Expression and Th2 Augmentation in Atopic Subjects.

Figure 4.2 Differential Effects of Selective Kinase Inhibitors on H22-Fel d 1Induced TSLPr Expression in Atopic and Non-Atopic moDCs. 


\section{List of Tables}

Table 2.1 Serum Antibody Profiles in Subjects With Distinct Immune Responses

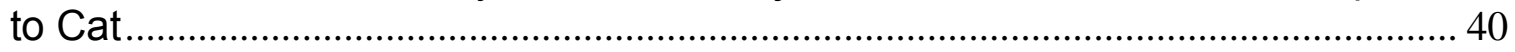

Table 3.1 Serum Antibody Profiles Associated With Distinct Allergic Phenotypes 80 


\section{Chapter I - Introduction}

Allergic sensitization is defined by the presence of $\operatorname{Ig} E A b$ to normally innocuous substances (i.e. allergens). $\mathrm{CD}^{+} \mathrm{T}$ cells are pivotal not only in the induction of $\mathrm{IgE}$, but also in the allergic inflammatory response. The main objective of this dissertation is to investigate the immunomodulatory properties of a novel allergen variant, $\mathrm{H} 22-\mathrm{Fel} \mathrm{d} 1$, at the $\mathrm{T}$ cell and dendritic cell level. This introductory chapter will begin with a review of some of the different types of allergic diseases, which include atopic dermatitis, asthma, and allergic rhinitis. We will then cover the role of type $2 \mathrm{CD}^{+} \mathrm{T}$ cells (Th2) in the initiation and maintenance of the allergic inflammatory response. We will also discuss what constitutes a protective $\mathrm{T}$ cell response in relation to allergy as well as some of the current strategies for inducing protive responses in allergic individuals. Finally we will discuss the features of $\mathrm{H} 22-\mathrm{Fel} \mathrm{d} 1$, which was designed to modulate T cell responses, and how an important Th2-promoting cytokine, TSLP, may influence these responses in vivo.

\section{Clinical Manifestations of Allergic Disease}

Allergic disorders include atopic dermatitis (eczema), asthma, and allergic rhinitis. The clinical signs of atopic dermatitis generally manifest in early life before the development of asthma and allergic rhinitis. In some individuals there is a sequential clinical progression of different types of allergic disease which has been referred to as the "atopic march". While atopic dermatitis may improve or dissipate with age, asthma or allergic rhinitis may subsequently develop and 
persist into adulthood (1). Several longitudinal studies from around the world have provided evidence for the atopic march (2-8). These studies have demonstrated that early allergic sensitization to foods or aeroallergens is a major risk factor for the development of asthma as an adult (2), and that the severity of atopic dermatitis in childhood correlates with the development of asthma later in life (4). It has been postulated that epicutaneous sensitization to allergens may activate cutaneous antigen presenting cells that then migrate to regional lymph nodes and prime naïve Th2 cells. These activated Th2 cells can then migrate throughout the body and subsequently reach other sites of allergen exposure such as the nasal and lung mucosa where they orchestrate allergic rhinitis and asthma respectively upon allergen encounter at those sites (1). A clearer understanding of the immune mechanisms involved in the generation of allergic $T$ cell responses, and how these responses may be modulated is pivotal to the design of new therapies.

\section{Atopic Dermatitis}

Atopic dermatitis is a chronic inflammatory skin disease associated with allergic sensitization to common environmental triggers (9), and is thought to represent a broad-based defect in immune regulation (10). This disease is diagnosed based on clinical criteria which include a characteristic distribution of age-specific itchy skin lesions $(1,8)$. Atopic dermatitis usually presents in early infancy and childhood, with a worldwide prevalence of $10-20 \%$, but can develop later in life and is present in $1-3 \%$ of adults (11). In children under two years of 
age, atopic dermatitis usually manifests as itchy, erythematous papules and vesicles on the cheeks, forehead or scalp. In children aged two years to puberty, skin lesions become lichenified owing to hyperkeratosis, and this is a common sign of chronic disease. Some patients present with an "acute-on-chronic" phenotype where new lesions arise on chronically affected skin. Typical areas of skin involvement in older patients include hands, feet, wrists, ankles, and the flexural folds of the elbow and knee. In adults, skin lesions are often dry and scaly, and can include large lichenified plaques, and in severe disease, the whole body may be involved. The distinguishing features of atopic dermatitis versus other allergic diseases are the markedly elevated levels of serum IgE and sensitization to a broad array of allergens (12).

The dermis of acute atopic dermatitis skin lesions is characterized by an infiltration of activated $\mathrm{CD} 4^{+}$memory $\mathrm{T}$ cells that express the skin homing marker cutaneous lymphocyte-associated antigen (CLA), and increased numbers of IL-4, IL-5, and IL-13-expressing cells compared to normal skin from healthy subjects (11). Antigen presenting cells in acute lesions, which include Langerhans cells (LC) and inflammatory dendritic epithelial cells (IDEC), have surface bound IgE. This facilitates capture of allergens and antigen presentation either in the skin or in regional nodes $(13,14)$. Chronic atopic dermatitis lesions are characterized by tissue remodeling, which results from a chronic inflammatory process. These lesions contain increased numbers of $\operatorname{lgE}^{+}$LCs and IDECs as well as eosinophils, and reduced numbers of T cells compared to acute lesions. Interestingly, chronic lesions have diminished IL-4- and IL-13-expressing cells, 
but enhanced levels of IL-5-, GM-CSF-, IL-12-, and IFN- $\gamma$-expressing cells, which is consistent with a role for Th1 cells in chronic disease (15). In both acute and chronic lesions, skin barrier function is disrupted, and this has been proposed to allow entry of allergens and microbial products that can trigger or exacerbate the allergic response.

\section{Asthma}

Asthma is a highly heterogeneous disease in which reversible airway obstruction is a pathognomonic feature. Manifestations of this disease range from a mild wheeze and breathlessness to a chronic corticosteroid-dependent phenotype that includes profound airway narrowing (16). Several cell types contribute to inflammatory responses in the asthmatic lung. These include CD4 ${ }^{+}$ T cells, eosinophils, neutrophils, and mast cells. Inflammation is generally restricted to the conducting airways, but can spread to the small airways and adjacent alveoli as the disease becomes more chronic and severe $(17,18)$. Clinical diagnosis of asthma is based on the patient's medical history, physical exam, assessment of airway obstruction reversibility and exclusion of other diseases which may mimic the asthma phenotype (19). In many patients asthma begins in childhood, and is the third leading cause of hospitalization for people under 18 in the United States (19). Asthma also develops during puberty or into adulthood, although many cases of adult onset asthma are due to a reappearance of symptoms in patients who were transiently symptom-free during adolescence. 
In patients with allergic asthma, inhalation of common allergens leads to bronchial constriction and increased mucus secretion that impairs breathing (airway hyperresponsivness (AHR)). Over time, these responses can lead to chronic disease and airway remodeling. This remodeling is characterized by airway smooth muscle thickening and development of fibrosis. It has been reported that in adult asthma the lung epithelium is chronically injured and cannot repair itself properly $(20,21)$. This results in reduced barrier function in the asthmatic lung, similar to what is seen in the skin of patients with atopic dermatitis. As a result, inhaled allergens, pollutants, and microbes can penetrate airway tissue (17). A dense network of subepithelial dendritic cells lines the respiratory tract. These cells are thought to be pivotal to priming $\mathrm{T}$ cell responses to allergens locally or in regional nodes.

\section{Allergic Rhinitis}

Allergic rhinitis is characterized by inflammation of the nasal mucosa associated with itching, sneezing and mucus secretion occurring upon exposure to allergen. Clinical diagnosis is based on the patient's medical history, positive immediate hypersensitivity skin test reactions to allergens and the presence of serum allergen-specific $\lg E \mathrm{ab}$, as well as anatomical assessments of the nasal cavity (22). Associated inflammatory cells include $\mathrm{CD} 4^{+} \mathrm{T}$ cells, eosinophils, mast cells and basophils. Allergic rhinitis is considered to be the most prevalent chronic respiratory disease, and as such, it is a worldwide public health issue (22). Depending on the frequency and intensity of symptoms, this disease can 
be classified as either intermittent or persistent and as either mild or moderate/severe.

Allergic rhinitis is a significant risk factor for the development of asthma $(23,24)$ and there is a close epidemiological relationship between allergic rhinitis and asthma. It has been suggested that the upper (nasal) and lower (lung) respiratory airways are a single anatomical and functional unit (25). As such, the nose functions as a barrier which filters and conditions air traveling to the lungs. When this barrier function is disrupted by allergic rhinitis, allergens not only have increased access to the nasal mucosa, but also to the lung (26). This is in keeping with the feature of impaired barrier function leading to allergic pathology in atopic dermatitis and asthma.

\section{Fel d 1: The Major Allergen From Cat}

This thesis focuses on the immunomodulatory properties of a molecule, which incorporates the major cat allergen Fel d 1, developed with a view to treating cat-allergic subjects. The domestic cat (Felis domesticus) is a common household pet as well as an important source of indoor allergens. The four known cat allergens include Fel d 1, Fel d 2 (cat albumin), Fel d 3 (cat cystatin), and Fel d 4 (27-30). Of these, Fel d 1 is the major allergen, and it accounts for up to $95 \%$ of the cat-specific IgE found in cat-allergic subjects (27). Fel d 1 is a $35 \mathrm{kDa}$ tetrameric glycoprotein composed of two heterodimer subunits (Figure 1.1)(31). Each heterodimer consists of two polypeptide chains which are 
Figure 1.1 Three-Dimensional Structure of Fel d 1 Indicating the Location of IL-10and IFN- $\gamma$-Inducing Epitopes.

Polypeptide chains 1 and 2 are shown in dark and light gray, respectively. The immunodominant region from chain 1 (P1:2) is shown in orange, and the chain 2 epitopes P2:1 and P2:2 are shown in yellow and blue, respectively. Overlapping residues from $\mathrm{P} 2: 1$ and $\mathrm{P} 2: 2$ are shown in green. Figure provided courtesy of $\mathrm{A}$. Pomes. 

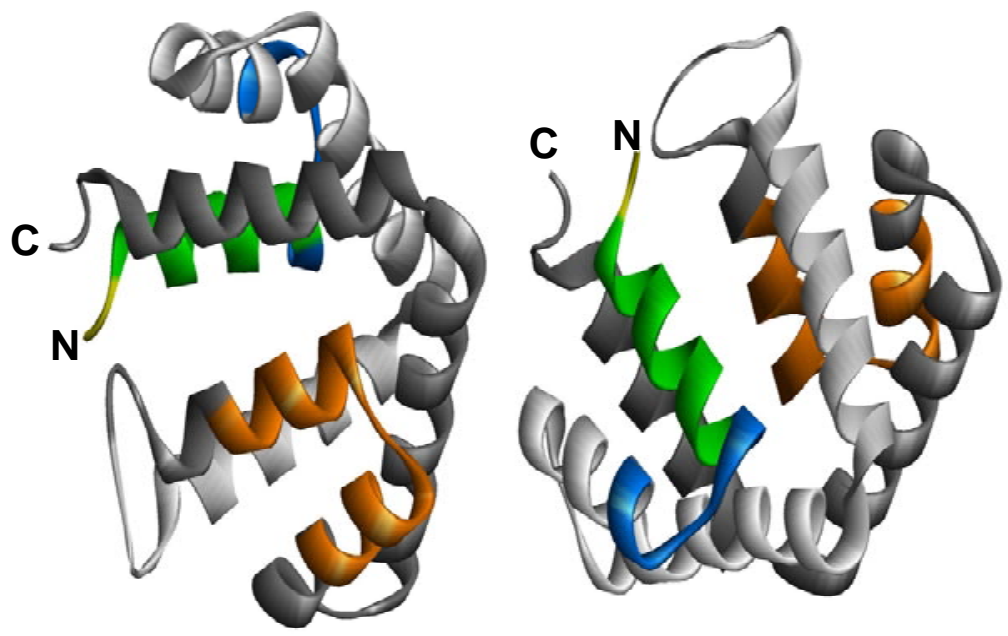
encoded by separate genes. Three disulfide bonds are formed between these two chains and are required for their linkage.

While the role of Fel $d 1$ in the cat is unknown, its structural similarities to proteins in the secretoglobin superfamily may provide insight to its function (32). Uteroglobin is one member of this family and is a steroid-inducible cytokine-like molecule that has potent anti-inflammatory and immunomodulatory properties (32-35). The protein Clara Cell 16 (CC16), another member of this family, is found in human lungs, and provides a protective effect against pathogens while also playing a role in controlling inflammation in this organ $(32-34,36,37)$.

Development of IgE antibodies to Fel d 1 results in symptoms ranging from mild rhinitis to severe asthmatic reactions. It is estimated that the prevalence of IgE-mediated sensitization to cat in the United States is as high as $17 \%$ (38). This high prevalence of sensitization coupled with the continuing increase in the prevalence of other allergic diseases has led to a significant burden on the health care systems of many nations.

Understanding the nature of T cell responses to Fel $\mathrm{d} 1$ is important for developing better therapeutic strategies. Unfortunately, Fel d 1 is a weak T cell stimulator in vitro and this has made evaluation of $\mathrm{T}$ cell responses to this allergen difficult in human-based systems. The next sections outline the role that T cells play in the allergic response and review our existing knowledge on t cell responses to Fel d 1. 
The Role of Th2 Cells in Allergic Inflammation

Chronic inflammation is a cardinal feature of allergic diseases. Allergenspecific type $2 \mathrm{CD}^{+} \mathrm{T}$ lymphocytes (Th2 cells) are central to the initiation and maintenance of the allergic inflammatory response through the cytokines they secrete (IL-4, IL-5, and IL-13)(39). IL-4 mediates antibody isotype switching to $\lg \mathrm{E}$. IgE binds the high affinity receptor, $\mathrm{Fc} \varepsilon \mathrm{RI}$, on the surface of mast cells, basophils, and eosinophils. Allergen-induced cross-linking of surface $\lg E$ on these cells leads to the release of inflammatory mediators including histamine and leukotrienes that perpetuate the allergic cycle. These mediators cause smooth muscle contraction and mucus production in the respiratory tract, or a wheal and flare reaction in the skin. The Th2-derived cytokines IL-5 and IL-13 are pivotal to other pathognomonic features of allergic disease such as eosinophil activation and migration as well as AHR. In addition, IL-4 and IL-13 are autocrine factors that stimulate Th2 differentiation and thus, amplify the allergic inflammatory response.

The pivotal role of Th2 cells in allergic disease has been established using murine models. For example, it has been shown that IL-4 knockout mice are resistant to allergen sensitization and do not develop eosinophilic lung infiltrates after subsequent allergen challenges (40). Similarly, IL-5 knockout mice show reduced eosinophilic lung infiltrates in response to allergen challenge and do not develop AHR (41). Moreover, several groups have demonstrated that passive transfer of Th2 cells from allergen-sensitized mice confers allergen-specific AHR in non-sensitized recipients (42-46). However, understanding the role of T cells 
in allergic disease in humans is challenging. Due to practical considerations, it is difficult to study the evolution of Th2 responses in early life. Established allergic responses are associated with a "mixed" $T$ cell infiltrate that includes Th1, Th2, Th0 and regulatory T cells (Treg), and represent only a snapshot in time of the progression of disease. In addition, it is not known why allergens preferentially promote Th2 responses in predisposed individuals.

Little is known about how Th2 responses develop in early life. Some have argued that transplacental priming to environmental allergens occurs in both lowrisk and high-risk infants, as evidenced by induction of proliferation in cord blood mononuclear cells derived from both groups after allergen challenge $(47,48)$. Furthermore, several epidemiologic studies have demonstrated that production of allergen-specific IgE antibodies begins as early as the first two years of life, but the immunological mechanisms responsible for this are unclear (39). It has been suggested that there is a universal propensity for Th2 skewing in neonates independent of allergen exposure arising from production of Th2 cytokines by the placenta, which protects the allogeneic fetus from the toxic effects of IFN- $\gamma$ and maternal Th1-mediated immunity (47). It has been demonstrated that PBMC from low-risk infants produced enhanced Th2 cytokine responses to allergen shortly after birth, but these responses declined with age (48). In contrast to this, infants at high-risk for developing atopy had decreased cytokine responses (both Th1 and Th2) to allergens and a non-specific T cell stimulus (PHA) shortly after birth, but their Th2 cytokine responses increased over time (as early as one year after birth). This may reflect defective control of Th2 responses in atopic children 
during a period when they are exposed to new allergens, although the genetic and/or environmental factors that provide the basis for their "predisposition" to atopy are still unclear.

The development of IgE-mediated sensitization and allergic disease in children across a wide age range suggests that memory Th2 responses continue to evolve over a prolonged time $(6,49,50)$. While it may be difficult to intervene with the initiation of Th2 skewing events that occur in utero or shortly after birth before the manifestation of allergic disease, strategies that modulate established Th2 responses in allergic individuals are a rational approach to treatment. In support of this, it is known that an established Th2 response is not a fixed state, but can be altered by therapeutic (immunotherapy) or environmental (change in allergen exposure) interventions (39).

\section{What Constitutes a Protective T Cell Response?}

This thesis focuses on the immunomodulatory properties of a molecule (H22-Fel d 1) developed with a view to treating cat-allergic subjects. In order to assess whether $\mathrm{H} 22-\mathrm{Fel} \mathrm{d} 1$ has therapeutic potential in cat-allergic subjects, it is important to consider what constitutes a protective T cell cytokine repertoire. Classical T cell paradigms hypothesize that Th1 and Th2 cells counter balance each other through the mutually inhibitory effects of the cytokines they secrete (i.e. IFN- $\gamma$ and IL-4 respectively). In the 1990's it was thought that treatments that induced Th1-skewing would promote the development of non-allergic responses. The recent consideration of Tregs has shifted this viewpoint. It is 
now generally accepted that induction of IL-10, which had originally been considered a Th2 cytokine, may suppress Th2 responses to allergens and thus be a more appropriate objective of therapy. Despite all that we have learned about $\mathrm{T}$-cell responses to allergens, including the role of Tregs, what defines a protective T-cell response to allergen still remains unclear.

The current T cell paradigm suggests that Tregs suppress Th2 responses to allergen (39). However, there are conflicting data on the nature of the Tregs which suppress allergic inflammation. Much of the work on Tregs within the immunology literature comes from murine models of disease. Many of these studies have focused on what have been defined as natural Tregs (nTregs). These cells are characterized by high level expression of the IL-2 receptor $\alpha$ chain, CD25, and the transcription factor Foxp3 (51). Natural Tregs develop in the thymus, and exert their regulatory effects in a cell contact-dependent manner. Another Treg population which also expresses Foxp3 and high levels of CD25 has been described. Unlike nTregs, these cells, termed adaptive Tregs (aTregs), are induced in the periphery and generally suppress through the action of secreted IL-10 or TGF- $\beta$ (51). The T regulatory 1 cell $(\operatorname{Tr} 1)$ is another subset of Tregs which is induced in the periphery and acts through IL-10, but does not express Foxp3. However, differences in the properties of murine and human Tregs make it difficult to accurately define a role for different Treg subsets in allergic disease. While Foxp3 expression appears to be a specific marker for Tregs in mice, this transcription factor is expressed in both nTregs and in activated effector T cells in humans (51). CD25 expression is also upregulated 
on activated effector $\mathrm{T}$ cells. Thus, Treg populations isolated from humans based on CD25 and Foxp3 expression may be contaminated with effector populations. In addition, because some subsets of Tregs, such as the Tr1 cells, do not have defined distinct surface phenotypes, additional markers of regulatory function are needed to isolate these populations for analysis. Recently, it has been demonstrated that expression of the IL-7 receptor $\alpha$ chain, CD127, is inversely correlated with both Foxp3 and in vitro suppressive function of human $\mathrm{CD}^{+}$Tregs (52-54). This finding could provide us with a more effective strategy for the isolation and functional analysis of purified Treg populations that may be induced by $\mathrm{H} 22-\mathrm{Fel} \mathrm{d} 1$.

\section{Evidence of "Tolerance" to Inhaled Allergens}

In 2001, Platts-Mills et al. reported a novel type of immune response (modified Th2 response) in children living with a cat who were exposed to high levels of the major cat allergen Fel d 1 (>4.4 $\mu \mathrm{g}$ Fel d 1/g of dust) (55). This response, which was characterized by the presence of anti-Fel d 1 lgG (including increased $\operatorname{lgG}_{4}$ ) antibodies in the serum without $\lg E$ and without allergic symptoms, was proposed to represent a form of high dose respiratory tolerance. This was a significant finding since the view at the time was that increasing levels of allergen exposure were associated with increasing prevalence of allergic sensitization and asthma (50). The modified Th2 response to Fel d 1 indicated that for some allergens, this linear dose-response relationship did not hold true at high levels of allergen exposure. Since that time, several studies have confirmed 
these observations suggesting that Fel $d 1$ has unique tolerogenic properties (56-59).

Subsequent T-cell studies performed in subjects with a modified Th2 response suggested a role for enhanced recognition of an immunodominant region within polypeptide chain 2 of Fel d 1 in the development of tolerance. This region contained T-cell epitopes that preferentially induced IL-10 and IFN- $\gamma$ (Figure 1.1) (60). T cells from subjects with a modified Th2 response who were ${\mathrm{HLA}-D R 7^{+}}^{+}$secreted higher levels of IL-10 in response to peptides spaning this region compared with both cat-allergic subjects and non-allergic controls (60). Moreover, T cells from patients receiving conventional immunotherapy using cat extract showed marked increases in IL-10 and IFN- $\gamma$ secretion after in vitro stimulation with Fel d 1 chain 2 peptides within four weeks of starting treatment (60). Collectively, these findings supported an association between IL-10 and IFN- $\gamma$ and protective responses to Fel $d 1$.

Immunomodulatory Effects of Immunotherapy

Allergen-specific immunotherapy (SIT) is a common therapeutic option for allergic patients. This treatment involves repeated subcutaneous injections, or else use sublingual application of extract containing allergens to which the patient is sensitized. Though extract is administered in an updosing regimen to minimize adverse effects, there are drawbacks. These include: a risk of IgEmediated adverse events; prolonged treatment regimens that require considerable patient compliance; and lack of standardization of allergen extracts 
that make it difficult to discern how much of the requisite immunogen is administered with each injection. Yet, in some settings, SIT has proven clinically efficacious for the treatment of allergic disease (61), and has been shown to improve quality of life by reducing symptoms and the need for medication (62). There is mounting evidence to suggest that the long term protective effects of immunotherapy are exerted at the T-cell level through induction of Tregs, which secrete IL-10 and/or Th1 cells, which secrete IFN- $\gamma(39,63-65)$.

Multiple studies have reported that SIT modifies responses of several effector cell types involved in allergic inflammation, including Th2 cells (66-69). It is known that $\mathrm{CD} 4^{+} \mathrm{T}$ cells from both allergic and healthy donors recognize the same allergen-specific T-cell epitopes (70-72). It has been suggested that it is the balance between allergen-induced IL-10-, IL-4-, and IFN- $\gamma$-secreting T cells that determines the development of a healthy versus allergic immune response (73). Several studies in humans that analyzed T cell responses directly ex vivo after SIT treatment suggest that SIT influences this balance by augmenting both allergen-specific $\mathrm{Tr} 1$-like and $\mathrm{CD} 4^{+} \mathrm{CD} 25^{+} \mathrm{nTreg}$ subsets (74-76). These regulatory $\mathrm{T}$ cells can then modify Th2 cell responses to allergen by inducing anergy (77) or suppressing effector responses $(76,78)$, and enhancing production of regulatory cytokines (IL-10 and TGF- $\beta)(63,73,74,78,79)$.

\section{Current Approaches to T Cell Modulation}

Due to adverse side effects involved with SIT, considerable effort has been devoted to the development of allergen-specific dendritic cell-based or T- 
cell-based vaccines, which may confer long-term protection without IgEmediated side effects. Allergen variants and allergen-derived peptides have been generated that lack conformational epitopes but retain the T-cell antigenic determinants necessary to activate $T$ cells $(80-85)$. In relation to peptide vaccines, peptides derived from allergens have been designed to retain linear $\mathrm{T}$ cell epitopes, without conformational B cell epitopes. Because of this, these peptides have a reduced capacity to cross-link allergen-specific IgE molecules, but can still stimulate allergen-specific T cells and induce protective responses (86). Initial T-cell epitope mapping studies of Fel d 1 revealed an immunodominant region of the molecule within polypeptide chain 1 (87). These studies led to the development of a peptide vaccine composed of two overlapping peptides (27 amino acids each) which spanned this region (88). While some improvement in lung and nasal symptom scores was seen in patients who received the highest does of the vaccine, numerous adverse events (mainly late asthmatic reactions) were also reported, which may have been due to retained B cell epitopes within the peptides based on their large size $(86,88)$. A similar study which used a mixture of shorter peptides from chain 1( 14-17 aminio acids) also induced late asthmatic reactions which appeared to be T-celldependent but IgE-independent (89). Despite this, decreased production of IL-4 or IL-5 and increased IL-10 secretion, along with decreased proliferation in allergen- and peptide-stimulated PBMC cultures, have been reported after peptide immunotherapy $(76,85,90-92)$. These data indicate there may be a clinical benefit associated with this approach. 
In work perfomed in Europe, allergen variants have been developed which exhibit reduced IgE binding, while retaining critical T-cell epitopes. Clinical trials using modified derivatives of the major birch pollen allergen, Bet $v 1$ (two recombinant Bet $\vee 1$ fragments and a Bet $v 1$ trimer), were performed by Valenta and colleagues (80). Treatment induced Bet $\vee 1$-specific IgG responses which could bind wild-type allergen and prevent its binding to $\lg E$ and induction of allergic symptoms $(80,93)$. In addition, treated patients noted an improvement of symptoms, and seasonally-induced Bet v 1-specific IgE was inhibited in these patients (93). While more trials are needed to confirm these results, it appears that immunotherapy with modified allergens holds promise as an alternate therapeutic option which may minimize the adverse side effects of conventional immunotherapy.

\section{Features of a Novel Allergen Variant Targeted to Dendritic Cells}

Allergen-specific $\mathrm{CD} 4^{+} \mathrm{T}$ cells recognize allergen-derived peptides (epitopes) presented in the context of major histocompatibility complex (MHC) class II molecules by APCs. As such, dendritic cells play a pivotal role in the initiation and maintenance of T-cell responses to allergens, both in health and in

disease. Specifically, the type of T-cell response generated can be influenced by the maturational state of dendritic cells. Engagement of a variety of surface receptors on dendritic cells can induce maturation, which is characterized by increased expression of an array of surface markers which are costimulatory for T cells (CD40, CD80, CD86, OX40L), as well as secretion of proinflammatory 
cytokines. Whereas mature dendritic cells generally induce effector T-cell responses (i.e. Th1 or Th2), immature or semi-mature dendritic cells are tolerogenic based on their ability to induce Tregs $(94,95)$. However, the situation is likely more complex as evidenced by recent reports that both mature and immature dendritic cells can induce Tregs (96).

Work performed in this thesis focuses on a variant of the major cat allergen Fel $d 1$ that is targeted to the high affinity receptor for IgG, Fc $\gamma \mathrm{RI}$ (CD64), which is expressed on dendritic cells. As demonstrated by the effects of high dose exposure to Fel $d 1$ and the development of a modified Th2 response, the nature of the T-cell response generated in response to any antigen is highly dependent on the dose of antigen administered. With this in mind, approaches that increase antigen delivery to the APC have the potential not only to enhance immunogenicity, but also to alter the quality of the T-cell response.

Dendritic cells express an array of Fc receptors that bind lgG. These include Fc $\gamma$ RI, Fc $\gamma$ RII and Fc $\gamma$ RIII. Whereas Fc $\gamma$ RI and Fc $\gamma$ RIII constitute activating receptors that contain ITAMs, Fc $\gamma$ RII exists in activating (Fc $\gamma$ RIla) and inhibitory (Fc $\gamma$ RIlb) forms. However, because this thesis focuses on the effects of targeting allergen to $\mathrm{Fc} \gamma \mathrm{RI}$, the next section will focus on the features of this particular receptor.

\section{Targeting Antigens to FC $\gamma R$ I}

The high affinity IgG receptor, Fc $\gamma \mathrm{RI}$ (CD64), is found exclusively on cells of the myeloid lineage. This receptor comprises an IgG-binding $\alpha$-chain 
complexed with an ITAM-containing $\gamma$-chain dimer. The ITAM within the $\gamma$ chain is critical to an array of FcyRI-mediated effector functions, including phagocytosis, cytokine production and antibody-dependent cell-mediated cytotoxicity. In addition to these functions, Fc $\gamma \mathrm{RI}$ also facilitates antigen presentation. However, though integrity of the $\gamma$-chain ITAM appears to be important for antigen presentation mediated by other Fc activating receptors, this may not be the case for FcyRI. Specifically, FcyRI has been reported to promote antigen presentation independently of a functional $\gamma$-chain ITAM (97). On the other hand, the $\alpha$-chain appears to be critical not only for antigen uptake, but also for antigen presentation through targeting of receptor-ligand complexes to the appropriate antigen processing compartments within the APC (97).

In the early 1990s, the effects of targeting antigen to Fc $\gamma R$ I were studied by conjugating antigens to the anti-human $\mathrm{Fc} \gamma \mathrm{RI}$ monoclonal antibody (mAb) 22.2. This mAb had been shown to bind to FcyRI via its Fab region outside the Fc binding domain, making it feasible to target antigens to the receptor despite occupancy by its normal ligand, IgG. Targeting antigens to human Fc $\gamma \mathrm{RI}$ using mAb 22 was shown to enhance T-cell responses in vitro in human-based systems and augment humoral responses in vivo in a transgenic mouse model $(98,99)$. Collectively, these findings pointed to enhanced immunogenicity mediated by FcyRI targeting using mAb 22. A humanized version of anti-CD64 mAb $22(\mathrm{H} 22)$ was developed, stimulated receptor internalization, an important first step in antigen processing (Figure 1.2) (100). This antibody cross-links 
Fc $\gamma \mathrm{RI}$ through binding by its Fc end as well as its Fab ends (101).

Interestingly, targeting antigenic peptides using just the monovalent form of $\mathrm{H} 22$ (Fab) enhanced both antigen specific $\mathrm{CD}^{+} \mathrm{T}$ cell proliferation and cytokine production in vitro (102). Using a similar approach to target prostate specific antigen to $\mathrm{Fc} \gamma \mathrm{RI}$ in the human monocytic cell line THP-1 led to enhanced killing of those cells by antigen-specific cytotoxic T lymphocytes (103). Thus, in addition to facilitating presentation of antigen on $\mathrm{MHC}$ class II molecules to $\mathrm{CD} 4^{+}$ T cells, exogenous antigens targeted to $\mathrm{Fc} \gamma \mathrm{RI}$ using $\mathrm{H} 22$ can also be presented to $\mathrm{CD}^{+} \mathrm{T}$ cells in the context of MHC class I. Moreover, a monovalent form of $\mathrm{H} 22$ is sufficient to mediate these effects.

The Novel Allergen Variant H22-Fel d 1

In 2002, Vailes et al. (104) developed a recombinant fusion protein, which linked Fel d 1 to a single-chain antibody fragment variable region (sFv) of $\mathrm{H} 22$ (H22-Fel d 1) (Figure 1.2). The sFv version of H22 (sFv22) binds monovalently and thus, does not crosslink Fc $\gamma \mathrm{RI}$, but nevertheless leads to receptor internalization. Interestingly, this effect requires IgG suggesting that occupancy of the ligand binding domain of $\mathrm{Fc} \gamma \mathrm{RI}$ is necessary for receptor uptake mediated by sFv22 (105). Subsequently, H22-Fel d 1 was shown to bind to FcyRI on monocytes. Moreover, H22-Fel d 1 retained the ability to bind Fel d 1-specific

\section{Figure 1.2 Schematic of H22-Fel d 1.}

A. The humanized anti-CD64 monoclonal antibody, $\mathrm{H} 22$, was generated by complementarity-determining region (CDR) grafting from the mouse 
monoclonal antibody (mAb) M22 (100). The humanized mAb contains $5-10 \%$ of the original mouse sequence. The sFv of $\mathrm{H} 22$ was linked to Fel $\mathrm{d} 1$ by cloning and H22-Fel d 1 was produced in Baculovirus and Pichia pastoris expression systems $(104,106)$. The sFv comprises the variable regions of heavy $(\mathrm{VH})$ and light (VL) chains of $\mathrm{H} 22$ produced by joining $\mathrm{VH}$ and VL DNAs together with a linker to obtain a single DNA fragment for cloning and expression. B. H22-Fel d 1 binds to FcyRI outside the Fc binding region of the receptor. 
A
M22
$\underline{\mathrm{H} 22}$
H22-Fel d 1
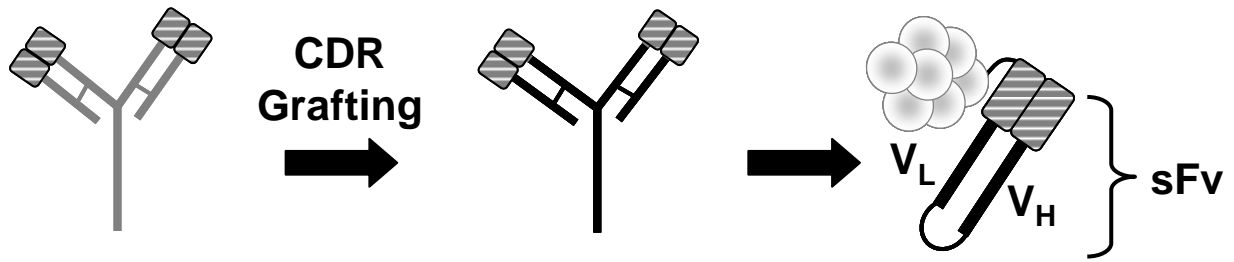

B

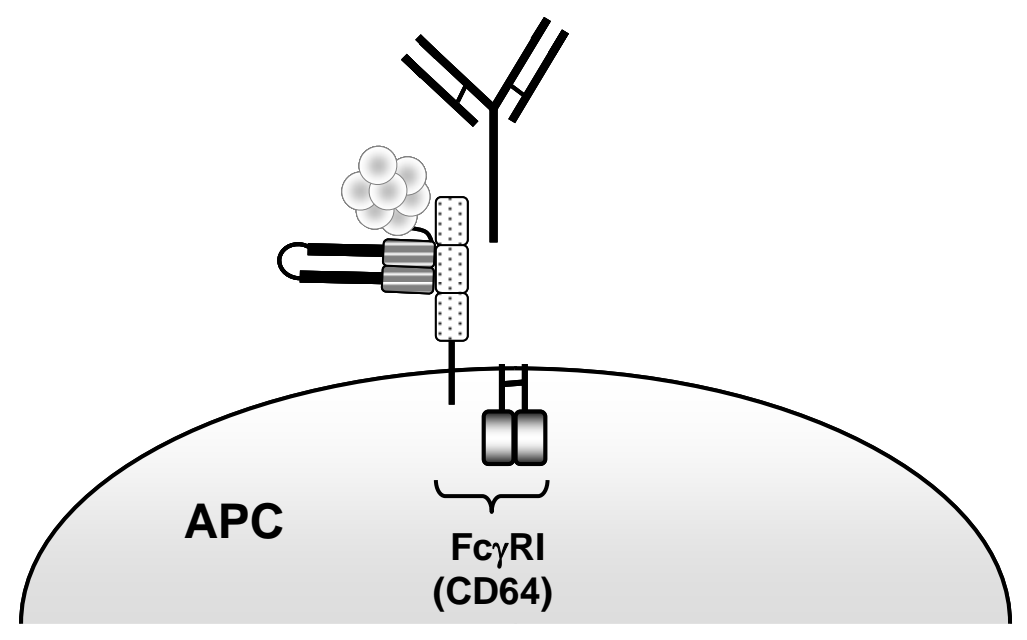


IgE antibody, suggesting that fusion of Fel d 1 to sFv22 did not influence allergen folding (104). This also raises the possibility that H22-Fel d 1 could cross-link IgE and IgG receptors, and play an important role in altering the signaling cascades induced in cells which express both types of these receptors. H22-Fel d 1 provides us with a novel molecular tool for studying the effects of targeting allergen to $\mathrm{Fc} \gamma \mathrm{RI}$ at the level of both the dendritic cell and the $\mathrm{T}$ cell in cat-allergic subjects. In addition to exploring its immunomodulatory properties, this thesis describes FcyRl-mediated events triggered by H22-Fel d 1 in dendritic cells. Prior to this work, the bulk of the work investigating the signaling events triggered by FcyRI has been performed in cells other than dendritic cells; these include primary human monocytes or macrophages, and an array of human and murine cell lines. Because of this, little is known about the mechanisms involved in FcyRI signaling in dendritic cells.

\section{Thymic Stromal Lymphopoietin - A Potential Immunomodulator In Vivo?}

Allergen variants that target dendritic cells in vitro do not act in isolation. Since dendritic cells are subject to the cytokine milieu in vivo, the nature of the T cell response induced by allergen variants may differ substantially from that observed in vitro. However, these microenvironmental effects, which are highly clinically relevant, are rarely addressed in in vitro culture systems. The cytokine thymic stromal lymphopoietin (TSLP) is expressed at high levels in the skin of patients with atopic dermatitis and in the asthmatic lung. This cytokine is a 
strong candidate for altering $\mathrm{T}$ cell responses induced by $\mathrm{H} 22-\mathrm{Fel} \mathrm{d} 1$ based on its Th2-polarizing properties and anatomical localization.

TSLP was originally characterized from the supernatant of a mouse thymic stromal cell line and subsequently identified as a four-helix bundle cytokine (107, 108). Human TSLP was discovered using database search methods, and had low homology $(43 \%)$ to murine TSLP $(109,110)$. The high affinity receptor for TSLP is a heterodimer consisting of the IL7-R $\alpha$ and TSLP receptor (TSLPr) (Figure 1.3). Binding of TSLP to this high affinity receptor on T cells and dendritic cells results in Stat5 activation and cellular proliferation $(109,111,112)$. TSLP is expressed by epithelial cells within the thymic medulla and mucosalassociated lymphoid tissues, as well as by keratinocytes of skin lesions from patients with atopic dermatitis and airway epithelial cells from asthmatic patients (109). This suggests that TSLP plays an important role both in T cell homeostasis and allergic inflammation, depending on where it is expressed (113).

Evidence that TSLP is involved in the generation of Th2 responses in mice has come from knock-out or overexpression models. Studies have demonstrated that TSLPr-deficient mice have attenuated Th2 responses, but normal Th1 responses (114). Moreover, mice with increased systemic TSLP expression have an increased number of IL-4-producing cells in addition to elevated levels of circulating $\lg E(115,116)$. These mice also spontaneously develop a condition similar to atopic dermatitis. It has also been proven that TSLP is both necessary and sufficient to induce airway inflammation in mice (117). In models of allergen- 
Figure 1.3 Schematic of the High Affinity TSLP Receptor.

The TSLP receptor (TSLPr) is a heterodimer composed of the IL-7R alpha chain and the TSLP receptor chain. The TSLP receptor chain is homologous to the common gamma chain. TSLPr is expressed on antigen presenting cells and a subset of activated Th2 cells in humans. 


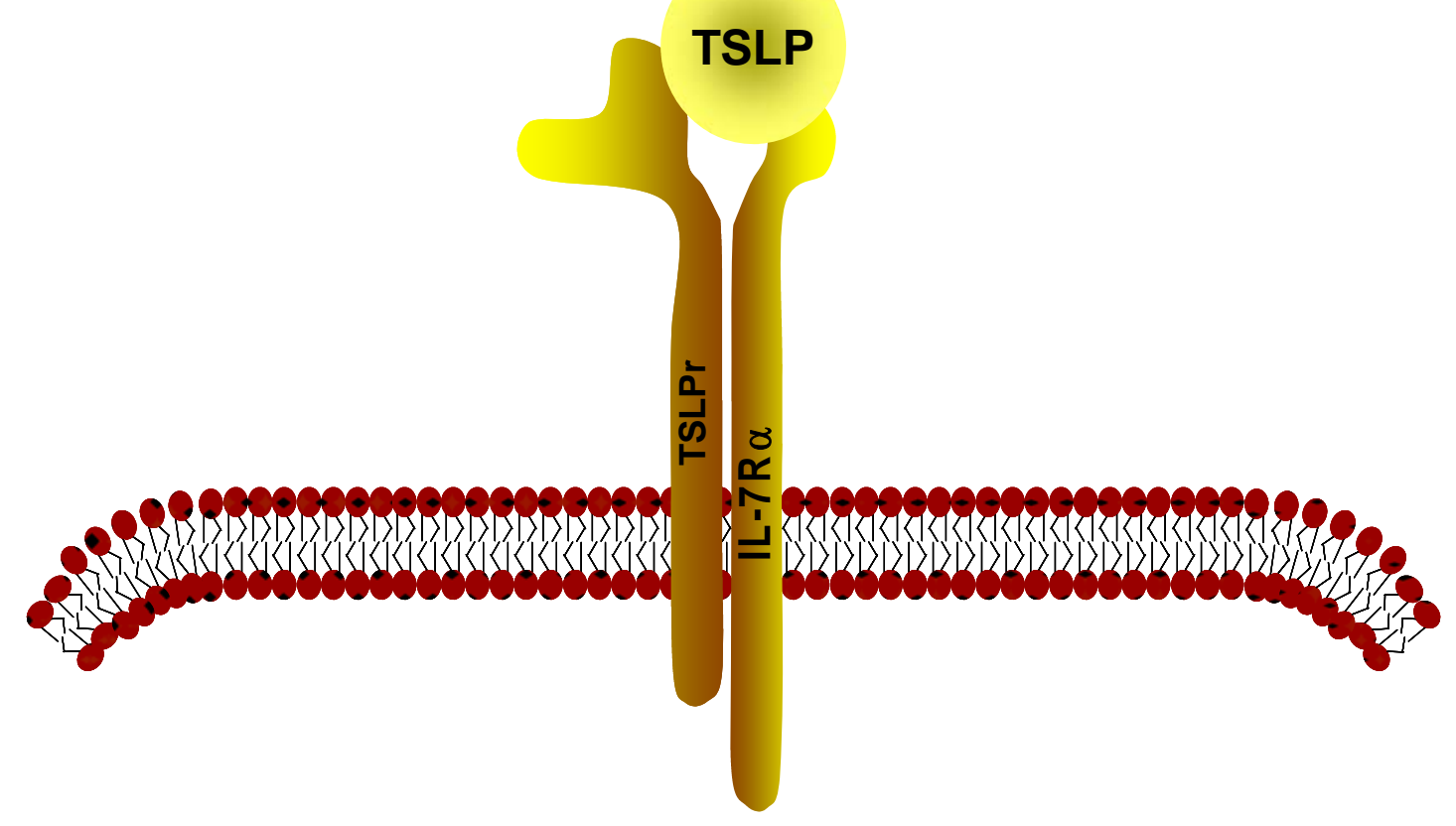


induced lung inflammation, TSLPr knock-out mice have significantly reduced disease after antigen challenge $(114,116)$. Conversely, increased TSLP expression in the lungs results in an airway-specific inflammatory disease which mimics human asthma (116). These murine models have provided important evidence that TSLP can induce inflammatory diseases with features similar to those found in human conditions, and may provide relevant models for studying these diseases.

Recent work has demonstrated that TSLP strongly enhances expression of MHC class II and co-stimulatory molecules on human dendritic cells (Figure 1.4). Unlike other conventional DC maturation stimuli, such as TLR ligands, TSLP-pulsed DCs do not express the Th1-polarizing cytokine IL-12. Instead, these DCs express the Th2-associated chemokines CXCL8 (IL-8), CCL24 (eotaxin-2), CCL17 (TARC), and CCL22) (MDC) (118). Furthermore, stimulation of naïve allogeneic $\mathrm{CD} 4^{+} \mathrm{T}$ cells by TSLP-pulsed DCs induces a unique Th2 cell that produces IL-4, IL-5, and IL-13 as well as TNF- $\alpha$, but not the regulatory cytokine IL-10, which is referred to as an inflammatory Th2 cell (118). Subsequently, it was shown that Th2 responses induced by TSLP-pulsed DCs occurred via an OX40L-dependent mechanism $(119,120)$. Since TSLP is expressed at the host/environment interface, it has been suggested that TSLP represents a "master allergic switch" in vivo. However, whether TSLP expression is triggered by environmental factors, including allergen, remains unclear.

In addition to its capacity to induce Th2 cells from naïve precursors, TSLP has been reported to play a role in the induction and maintenance of allergen- 
Figure 1.4 Effects of TSLP on Human Dendritic Cells.

TSLP strongly enhances expression of MHC class II and co-stimulatory molecules on human dendritic cells. TSLP-pulsed DCs do not express the Th1polarizing cytokine IL-12. Instead, these DCs express the Th2-asscociated chemokines IL-8, eotaxin-2, TARC, and MDC. TSLP-pulsed DCs induce a unique Th2 cell that produces IL-4, IL-5, and IL-13 as well as TNF- $\alpha$, but no IL10 , which is referred to as an inflammatory Th2 cell. 


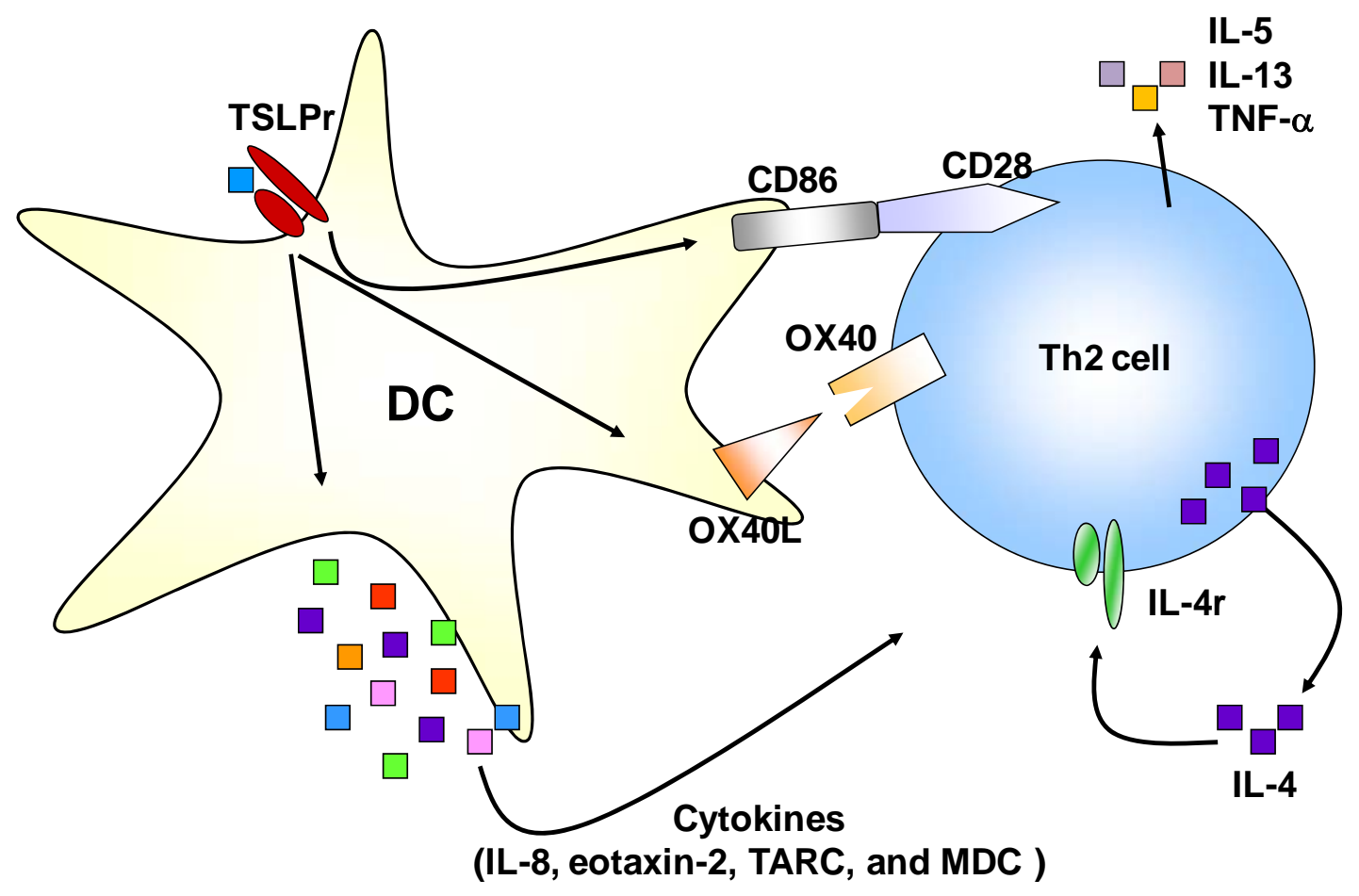


specific memory Th2 cells. In humans, memory $\mathrm{CD}^{+} \mathrm{T}$ cells can be divided into two distinct subsets based on their surface phenotype, homing capacity and effector functions. Central memory cells $(\mathrm{Tcm})$ are $\mathrm{CD} 45 \mathrm{RO} \mathrm{CCR}^{+} \mathrm{CD} 62 \mathrm{~L}^{+}$, can traffic to draining lymph nodes and undergo homeostatic proliferation. Effector memory cells (Tem) are $\mathrm{CD} 45 \mathrm{RO}^{+} \mathrm{CCR} 7 \mathrm{CD}^{-} \mathrm{CL}^{+/-}$, are found in peripheral sites, and can rapidly gain effector function (121-123). These subsets can be further divided based on their propensity to produce Th1- or Th2-associated cytokines, and differential expression of homing receptors (124-126). Recently, Liu and colleagues demonstrated that TSLP-pulsed dendritic cells maintain a distinct subset of Tcm which express the Th2-associated marker chemoattractant receptor-homologous molecule expressed on Th2 cells (CRTH2) (124). In addition, they showed that this maintenance was dependent on OX40/OX40L interactions at the $\mathrm{T}$ cell/dendritic cell interface. Importantly, $\mathrm{CRTH} 2^{+} \mathrm{T}$ cells were localized to skin lesions of patients with atopic dermatitis, but not lesions from patients with psoriasis (a Th1-associated disease) or non-lesional skin from atopic dermatitis patients (124). It was further demonstrated that TSLP could enhance the Th2 properties of $\mathrm{CRTH}_{2}{ }^{+} \mathrm{T}$ cells by leading to the upregulation of IL-17RB, the receptor for IL-25 (IL-17E) (127).

With these properties in mind, a major objective of this thesis was to investigate the capacity for TSLP to modulate T cell responses induced by $\mathrm{H} 22-$ Fel d 1. An important aspect of our studies was to examine whether TSLP exerted a differential effect depending on allergic status. This is pertinent, since previous work that reported the Th2-promoting properties of TSLP using human- 
based in vitro systems did not take into account the allergic status of donors used for isolating dendritic cells (119). Our findings provide evidence of a differential role for TSLP depending on allergic status and establish a link between molecular events triggered by $\mathrm{H} 22-\mathrm{Fel} \mathrm{d} 1$ and Th2 responses mediated by the TSLP pathway in human dendritic cells.

\section{Goals of the Dissertation}

\section{Aim 1: Analysis of the Immuomodulatory Effects of H22-Fel d 1.} Hypothesis: H22-Fel d 1 induces a $T$ cell cytokine repertoire with protective features.

A major aim of allergen immunotherapy is to induce allergen-specific protective responses at the $T$ cell level. Exposure to high dose cat allergen (Fel d 1), either through natural exposure in the environment or through conventional immunotherapy, is associated with development of protective T cell responses. We theorized that enhancing delivery of cat allergen to the antigen presenting

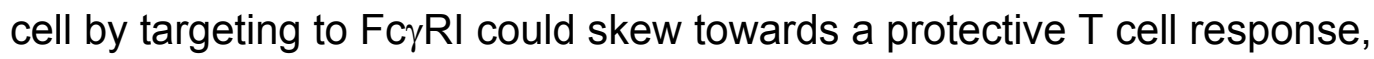
possibly by mimicking high dose natural allergen exposure. There is evidence that targeting antigens by this approach enhances antigen delivery to the antigen presenting cell and augments $\mathrm{T}$ cell responses. However, nothing is known about the immunomodulatory effects of targeting allergens to Fc $\gamma \mathrm{RI}$. Many of the previous in vitro studies that have investigated the T cell modulatory effects of conventional immunotherapy, or else of allergen variants or peptide vaccines, used bulk culture systems that do not yield information regarding qualitative 
changes that occur at the single cell level. The first goal of this dissertation is to examine the immunomodulatory effects of targeting cat allergen to Fc $\gamma \mathrm{RI}$ on dendritic cells, by analyzing the T cell cytokine repertoire at the single cell level. Initial studies will focus on investigating whether $\mathrm{H} 22-\mathrm{Fel} \mathrm{d} 1$ induces a protective phenotype in moDCs from cat-allergic subjects as judged by cytokine production and expression of surface co-stimulatory molecules. In order to investigate the effects of H22-Fel d 1 at the T cell level, co-cultures of H22-Fel d 1-stimulated moDCs and $\mathrm{CD}^{+} \mathrm{T}$ cells will be established. Intracellular cytokine staining with flow cytometry analysis will then be used to determine whether $\mathrm{H} 22-\mathrm{Fel} \mathrm{d} 1$ induces qualitative changes in the T cell cytokine repertoire which incorporate elements of a protective response. Cell cultures will also be analyzed from nonallergic subjects (modified Th2 responders and serum antibody negative controls) in order to determine whether there is a differential effect of $\mathrm{H} 22-\mathrm{Fel} \mathrm{d} 1$ on $\mathrm{T}$ cell responses based on allergic status. The $\mathrm{T}$ cell cytokine repertoire induced by $\mathrm{H} 22-\mathrm{Fel} \mathrm{d} 1$ will also be compared to the repertoire induced by nonreceptor targeted allergens, including Fel d 1 and the prototypic dust mite allergen Der $\mathrm{p} 1$.

Aim 2: Analysis of the Effects of TSLP on T Cell Responses Induced by H22-Fel d 1. Hypothesis: H22-Fel d 1 and TSLP coordinate to enhance Th2 responses in atopic subjects. 
In the in vivo setting, dendritic cells targeted by $\mathrm{H} 22-\mathrm{Fel} \mathrm{d} 1$ will be subject to the influence of the cytokine milieu. Thus, the nature of the $\mathrm{T}$ cell response induced by $\mathrm{H} 22-\mathrm{Fel} \mathrm{d} 1$ in vivo may differ substantially from that observed ex vivo. Such microenvironmental effects, which are clinically relevant, are rarely addressed in in vitro culture systems. We postulate that the clinical efficacy of H22-Fel d 1 will depend on its capacity to induce a protective T cell cytokine repertoire in a "pro-allergic" cytokine milieu. The Th2-promoting cytokine, TSLP, acts directly on dendritic cells and is pivotal to the induction of Th2 responses. This cytokine is expressed at sites of allergic inflammation, including sites commonly used to administer immunotherapy (eg. skin). Thus, in allergic subjects, exposure of dendritic cells to TSLP could mitigate the protective T cell elements induced by $\mathrm{H} 22-\mathrm{Fel} d \mathrm{~d}$. With this in mind, we will test whether H22-Fel d 1 and TSLP coordinate to enhance Th2 responses in cat-allergic subjects. An important aspect of these studies will be to examine whether there are differential effects of TSLP depending on atopic status, since this is not known. Initially, we will examine whether TSLP alone induces Th2 responses in atopic and nonatopic subjects. Next, the T cell cytokine repertoire induced by H22-Fel $\mathrm{d} 1$ in the presence of TSLP will be compared to that induced by H22-Fel d 1 or TSLP alone. Secreted cytokines will be measure to confirm intracellular cytokine patterns observed by flow cytometry.

\section{Aim 3: Elucidation of the Molecular Events Induced by H22-Fel d 1 That} Promote the TSLP Pathway. 
Hypothesis: Targeting Fel d 1 to Fc $\gamma R I$ on dendritic cells triggers intracellular signaling events which enhance the TSLP pathway.

The final aim of this dissertation is to elucidate the molecular mechanisms which mediate amplification of Th2 responses in atopic dendritic cells costimulated with H22-Fel d 1 and TSLP. Studies are designed to identify differences in atopic and non-atopic dendritic cells that could account for Th2 amplification. Little is known about the TSLP signaling pathway in human dendritic cells. However, because Th2 responses induced by TSLP were previously reported to be OX40L-dependent, we will first investigate whether this pathway is required for Th2 amplification. To do this, we will examine whether surface expression of $\mathrm{OX} 40 \mathrm{~L}$ is increased on dendritic cells by stimulating with H22-Fel d 1 in the presence or absence of TSLP. In conjunction, the effects of OX40L blockade on $\mathrm{T}$ cell responses will be analyzed. We will also investigate whether other factors, such as secreted cytokines or surface molecules are involved in Th2 amplification. Finally, we will examine whether targeting $\mathrm{H} 22-\mathrm{Fel}$ d 1 to $\mathrm{Fc} \gamma \mathrm{RI}$ amplifies the TSLP pathway through upregulation of TSLP receptor. It is well established that cytokine pathways can modulate expression of Fc receptors. However, discovery of a pathway linking Fc $\gamma R$ I to TSLPr would provide a novel finding. Initially we will compare the ability for $\mathrm{H} 22-\mathrm{Fel} \mathrm{d} 1$ to upregulate TSLPr expression in dendritic cells from atopic and non-atopic subjects. The requirement for FcyRI-mediated signaling events in the expression of TSLPr will be investigated using kinase inhibitors. Finally, the capacity for these inhibitors to inhibit TSLP-driven Th2 responses triggered by H22-Fel d 1 
will be examined in order to confirm a role for FcyRI-mediated signaling events in Th2 amplification. 


\section{Chapter II - Targeting Fel d 1 to Fc $\gamma R$ I Induces a Novel Variation of the Th2 Response in Subjects with Cat Allergy}

\section{Introduction}

Conventional immunotherapy, which involves repeated injection of allergen extracts, remains the mainstay of treatment for allergy to a variety of inhalant allergens. Although there is considerable evidence for its efficacy, this approach has several drawbacks, including a risk for anaphylaxis and prolonged treatment regimens. Although the immune mechanisms which underlie the protective effects of immunotherapy remain ill defined, there is evidence that induction of $\mathrm{CD}^{+} \mathrm{T}$ cells, which secrete IL-10 or IFN- $\gamma$, is a prerequisite. This suggests the involvement of T cells that are either regulatory or Th1-like $(60,74$, $79,128)$. Consequently, recombinant allergens or allergen variants, which may provide a more tailored approach to immunotherapy, have been engineered with a view to activating specific T-cell subsets preferentially $(74,129,130)$. Unfortunately, many allergens induce weak T-cell responses in vitro, which may reflect the low precursor frequency of allergen-specific T cells in the T-cell repertoire. As a result, defining T-cell mechanisms that underlie the protective effects of immunotherapy has proved to be a challenge. Moreover, in vivo presentation of allergen to $\mathrm{T}$ cells during conventional immunotherapy is likely to be inefficient.

The approach described here uses a fusion protein designed to target allergen to the surface of antigen-presenting cells (APCs) as a method of 
improving allergen presentation to T cells. This protein (designated H22-Fel d 1 ) is composed of the major cat allergen, Fel d 1, linked to a single chain fragment of the variable region (sFv) of the humanized anti-CD64 mAb, H22. This molecule has been shown to bind to the high-affinity IgG receptor, Fc $\gamma R \mathrm{I}$ (CD64), on APCs (104). Targeting to CD64 has been demonstrated to increase uptake of diverse antigens by APCs and to increase T-cell proliferative responses $(98,102,104,131)$.

A unique feature of the major cat allergen, Fel $d 1$, is its ability to induce a modified Th2 response $\left(\lg \mathrm{G}^{\text {pos }} \mid g \mathrm{E}^{\text {neg }}\right)$ in a subset of individuals with high-level environmental exposure (55). This protective response is associated with increased IL-10 production in PBMC cultures stimulated with major T-cell epitopes of Fel d 1 (60). Because targeting Fel d 1 to CD64 on APCs may enhance presentation of major T-cell epitopes, we hypothesized that H22-Fel d 1 would promote induction of IL-10-producing $\mathrm{CD} 4^{+} \mathrm{T}$ cells. These studies were designed to examine the immunomodulatory effects of H22-Fel d 1 at both the APC and the T-cell level. To this end, we analyzed patterns of cytokine secretion by dendritic cells and performed rigorous single-cell analyses of cytokine production by $\mathrm{CD} 4^{+} \mathrm{T}$ cells. Our findings provide evidence of a new approach to immunotherapy and present a new paradigm for variations of a Th2 response. 


\section{Materials and Methods}

\section{Classification of Subjects}

Patients were recruited from the University of Virginia Allergic Diseases Clinic or by advertisement. Subjects with cat allergy were classified on the basis of high-titer serum IgE antibodies to cat extract (CAP $>0.7 \mathrm{IU} / \mathrm{mL}$ ) and anti-Fel d $1 \mathrm{lgE}$ antibodies measured by streptavidin CAP assay (132). Subjects with a modified Th2 response to cat allergen were identified on the basis of high-titer anti-Fel d 1 IgG antibodies measured by antigen binding radioimmunoassay (>500 U/mL) without anti-Fel d $1 \mathrm{lgE}$ antibodies $(55,133)$. Control subjects had no measurable serum $\lg G$ or $\lg E$ antibodies to Fel d 1 . Serum antibody profiles are shown in Table 2.1. All studies were approved by the University of Virginia Human Investigations Committee.

Recombinant Allergens and LPS

Recombinant Fel d 1 and Fel d 1 targeted to the high-affinity IgG receptor, FcyRI (H22-Fel d 1) (104), were expressed in Pichia pastoris and purified by multistep chromatography. The purity was $>90 \%$ as determined by SDS-PAGE. The endotoxin content of Fel d 1 and H22-Fel d 1 was comparable (145 EU/mL and $153 \mathrm{EU} / \mathrm{mL}$, respectively). LoTox Der p 1 (Indoor Biotechnologies Inc, Charlottesville, Va) (endotoxin content $<0.05 \mathrm{EU} / \mathrm{mg}$ ) was purified from mite culture by affinity chromatography (>90\% purity by SDS-PAGE) and endotoxin removed by proprietary techniques. LPS was obtained from Sigma-Aldrich (St Louis, Mo). 
Table 2.1 Serum Antibody Profiles in Subjects With Distinct Immune

\section{Responses to Cat}

\begin{tabular}{|c|c|c|c|c|}
\hline \multirow[b]{2}{*}{ Group } & \multirow[b]{2}{*}{$\begin{array}{c}\text { Total IgE }^{1} \\
\text { (IU/ml) }\end{array}$} & \multirow[b]{2}{*}{$\begin{array}{c}\text { IgE to Cat } \\
\text { (IU/ml) }\end{array}$} & \multicolumn{2}{|c|}{ Fel d 1} \\
\hline & & & $\begin{array}{c}\mathrm{IgE}^{2} \\
\text { (IU/ml) }\end{array}$ & $\begin{array}{c}\mathrm{IgG}^{3} \\
(\mathrm{U} / \mathrm{ml})\end{array}$ \\
\hline Allergic $(\mathrm{n}=11)$ & $\begin{array}{c}119 \\
(60-235)\end{array}$ & $\begin{array}{c}13 \\
(7-25)\end{array}$ & $\begin{array}{c}7 \\
(0.5-99)\end{array}$ & $\begin{array}{c}3168 \\
(1392-7213)\end{array}$ \\
\hline Modified Th2 (n=9) & $\begin{array}{c}22 \\
(10-46)\end{array}$ & $<0.35$ & $<0.35$ & $\begin{array}{c}4917 \\
(1923-12573)\end{array}$ \\
\hline Control (n=5) & $\begin{array}{c}14 \\
(4-45)\end{array}$ & $<0.35$ & $<0.35$ & $<125$ \\
\hline
\end{tabular}


Proliferation Assays

PBMCs were cultured for 7 days $\left(2 \times 10^{5}\right.$ cells/well in $96-$ well plates $)$ in the presence of Fel d 1 or $\mathrm{H} 22-F e l$ d $1(0.1,1$, or $10 \mu \mathrm{g} / \mathrm{mL} ; \geq 6$ replicate wells per condition). Cells were pulsed with $1 \mu \mathrm{Ci}$ of $\left[{ }^{3} \mathrm{H}\right]$ thymidine/well during the final $8 \mathrm{~h}$ of culture before harvesting and counting (Topcount NXT; Packard Instrument, Meriden, CT).

Induction of cytokines in monocyte-derived dendritic cells

CD14 ${ }^{+}$monocytes were purified by magnetic-activated cell sorting (Miltenyi Biotec, Auburn, Calif) and cultured for 6 days in RPMI-1640 with 10\% heat-inactivated FBS, L-glutamine, $100 \mathrm{U} / \mathrm{mL}$ penicillin, $100 \mathrm{mg} / \mathrm{mL}$ streptomycin, $1 \%$ sodium pyruvate, $100 \mathrm{ng} / \mathrm{mL}$ recombinant human GM-CSF, and $100 \mathrm{ng} / \mathrm{mL}$ recombinant human IL-4 (BioSource International, Inc, Carlsbad, Calif) to generate monocyte-derived dendritic cells (moDC) (134). Half of the media in each well was changed every 2 to 3 days. moDCs were stimulated for 24 to 48 hours in medium containing $10 \%$ autologous human serum with allergen (10 $\mu \mathrm{g} / \mathrm{mL})$ or LPS $(1 \mu \mathrm{g} / \mathrm{mL})$. Cytokines were measured by cytometric bead assay (human 27-plex kit; BioRad, Hercules, CA) or ELISA (DuoSet Human CCL17/TARC; R\&D Systems, Minneapolis, MN) according to the manufacturer's instructions.

CD4 ${ }^{+}$T-cell/moDC co-cultures

$\mathrm{CD}^{+} \mathrm{T}$ cells were purified by negative selection using magnetic-activated cell sorting (Miltenyi Biotec, Auburn, Calif), and purity was assessed by flow 
cytometry (>95\%). moDCs were pulsed with allergen $(10 \mu \mathrm{g} / \mathrm{mL})$ for 48 hours before coculture $\left(2 \times 10^{5}\right)$ with purified $\mathrm{CD}^{+} \mathrm{T}$ cells $\left(8 \times 10^{5}\right)$ for 10 days in 24well plates. Cultures were supplemented with recombinant human IL-2 (12 U/mL; BioSource International, Carlsbad, Calif) on day 5, and intracellular cytokines were measured on day 10. In some experiments, a blocking anti-IL-10 receptor mAb (clone 3F9; BioLegend, San Diego, Calif) or isotype control (clone RTK2758; BioLegend) was added (20 $\mu \mathrm{g} / \mathrm{mL})$ on day 0 of coculture.

\section{Flow Cytometry}

All flow cytometry was performed on a Becton Dickinson FACSCalibur machine equipped with CellQuest software version 5.2 (BD Biosciences, Mountain View, Calif) and upgraded with a 635-nm red diode laser run by Rainbow software version 1.2b3 (Cytek Development, Gordonsville, Va). All experiments were analyzed by using FlowJo version 6.4.1 (Tree Star Inc, Ashland, Ore).

Unless otherwise stated, all fluorescently conjugated mAbs were purchased from BD Biosciences. moDC surface mAbs included phycoerythrinanti-CD1a (HI149), peridinin-chlorophyll-protein-anti-CD14 (MuP9), APC-antiCD11c (B-ly6), biotin conjugated anti-CD64 (10.1) then avidin-APC-Cy7, and fluorescein isothiocyanate-anti-HLA-DR (L2234), anti-CD40 (5C3), anti-CD80 (L307.4), or anti-CD86 (2331). T-cell surface mAbs included peridininchlorophyll-protein-anti-CD8 (SK1) and APC-Cy7-anti-CD3 (SK7), and 
intracellular mAbs included fluorescein isothiocyanate-anti-IL-5 (R\&D Systems Inc, 22026, Minneapolis, Minn), phycoerythrin-anti-IL-10 (JES3-9D7), and APCanti-IFN- $\gamma$ (25723.11). For all multicolor analyses, compensation controls (single stains, 1 for each fluorochrome) and gating controls (cells stained with all reagents minus 1) were included (135).

moDC Phenotyping

Monocyte-derived dendritic cells were collected immediately after generation or allergen pulsing (24-48 hours) and washed in cold FACS buffer (0.5\% BSA, $2 \mathrm{mmol} / \mathrm{L}$ EDTA in PBS). Cells were stained for surface markers, washed, and analyzed by flow cytometry as described above.

Intracellular cytokine staining

$\mathrm{CD}^{+} \mathrm{T}$-cell/moDC cocultures were restimulated with phorbol 12-myristate 13-acetate (50 ng/mL) and ionomycin (2 $\mu \mathrm{g} / \mathrm{mL}$; Sigma-Aldrich) for 5 hours, and Brefeldin A (BD Biosciences, San Jose, Calif) was added during the final 4 hours. Cells were stained for surface and intracellular cytokines according to the manufacturer's instructions (Caltag International Inc, Carlsbad, Calif) and analyzed by flow cytometry as described above.

Statistical analysis

The Student t test (SPSS 14.0, SPSS Inc, Chicago, III) was used to compare proliferation, cytokines, and mean fluorescence intensity levels. The percentages of cytokine-positive $\mathrm{CD}^{+} \mathrm{T}$ cells induced by allergen were analyzed 
by general linear mixed-effects ANOVA models after transforming data to the natural logarithmic scale. Each ANOVA model was specified as a hierarchical model with respect to the model factors. For each ANOVA, the stimulus and cytokine type represented the highest and lowest level factor of the 2-level hierarchical model, respectively. Data from each subject represented an independent random block of correlated information within the model. ANOVA model parameters were estimated via restricted maximum likelihood, and the variance components were modeled in the compound symmetry variancecovariance matrix form. Linear contrasts of the ANOVA least-squares means were used to analyze the log-transformed data. All hypothesis tests were 2sided, and the Tukey multiple comparison type I error rate adjustment was used so that the cumulative type I error rate $(\alpha)$ was $\leq 0.1$ for the entire set of hypothesis tests. This value was selected on the basis of the conservative nature of the Tukey adjustment. The models were fit using PROC MIXED in SAS, version 9.1 (SAS Institute Inc, Cary, NC).

\section{Results}

H22-Fel $d 1$ induces production of inflammatory cytokines by dendritic cells without increasing surface markers of maturation

Dendritic cells are essential for generating T-cell responses to allergens. These responses may be influenced by the degree of maturation of the dendritic cell as well as by the cytokines these cells secrete $(124,129,136)$. We investigated whether binding of $\mathrm{H} 22-\mathrm{Fel} \mathrm{d} 1$ to $\mathrm{Fc} \gamma \mathrm{RI}$ on dendritic cells alters the 
properties of these cells. Flow-cytometry analysis showed that moDCs obtained from subjects with cat allergy expressed a phenotype characteristic of myeloid dendritic cells including high-level expression of CD11c, CD1a, and HLA-DR and lack of expression of CD14 (Figure 2.1). Importantly, these cells also expressed CD64 (Figure 2.2). As expected, stimulation with LPS led to increased expression of the costimulatory molecules CD40, CD80, and CD86 on moDCs, consistent with maturation, whereas expression of CD64 was decreased $(p \leq .01)$. However, stimulation with H22-Fel d 1 or with Fel d 1 had no significant effect on these markers (Figure 2.3). By contrast, H22Fel d 1 induced increased secretion of Th1-promoting and inflammatory cytokines (IL-12, RANTES, IL-1 $\beta$, monocyte chemoattractant protein (MCP)-1, macrophage inflammatory protein (MIP)-1 $\beta$, MIP-1 $\alpha$ ) by moDCs compared with Fel d $1(p<.05)$. Moreover, levels for selected inflammatory cytokines (MCP-1, MIP-1 $\beta$ ) were comparable to those induced by LPS (Figure 2.4). H22-Fel d 1 also induced increased IL-10 compared with Fel d $1(p=.04)$, whereas no change in the levels of Th2-attracting chemokines (thymus and activation-regulated chemokine (TARC) and eotaxin) was observed for any stimuli (Figure 2.4, and data not shown). Induction of inflammatory cytokines in dendritic cells by H22Fel d 1, without increased expression of maturation markers, indicates a semimature phenotype (137). 
Figure 2.1 MoDC phenotype.

MoDCs were generated from CD14 ${ }^{+} \mathrm{T}$ cells by culturing in the presence of IL-4 and GM-CSF for 6 days. Representative flow cytometry plots from 1 of 6 allergic subjects demonstrating the $C D 11 c^{+}, C D 14^{\text {neg }}$ phenotype of moDCs after 6 days in culture with IL-4 and GM-CSF. 

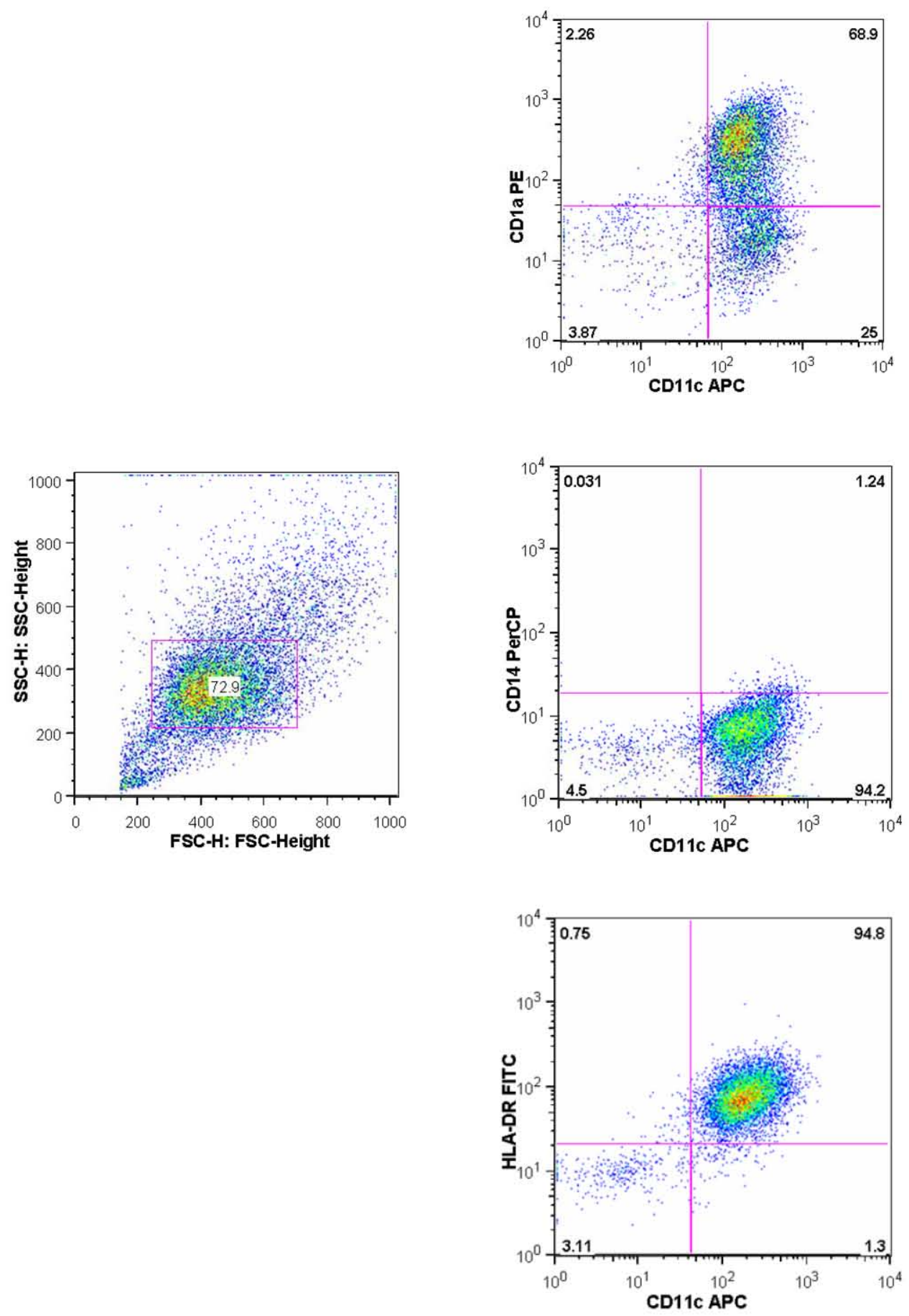
Figure 2.2 CD64 is Expressed on MoDCs.

MoDCs from subjects with cat allergy $(n=6)$ were pulsed with antigen (24 hours) and analyzed for expression CD64 by flow cytometry. A. Representative data showing expression of CD64 after 6 day generation of moDCs. B.

Representative data showing expression of CD64 after 24 hour antigen pulse. 

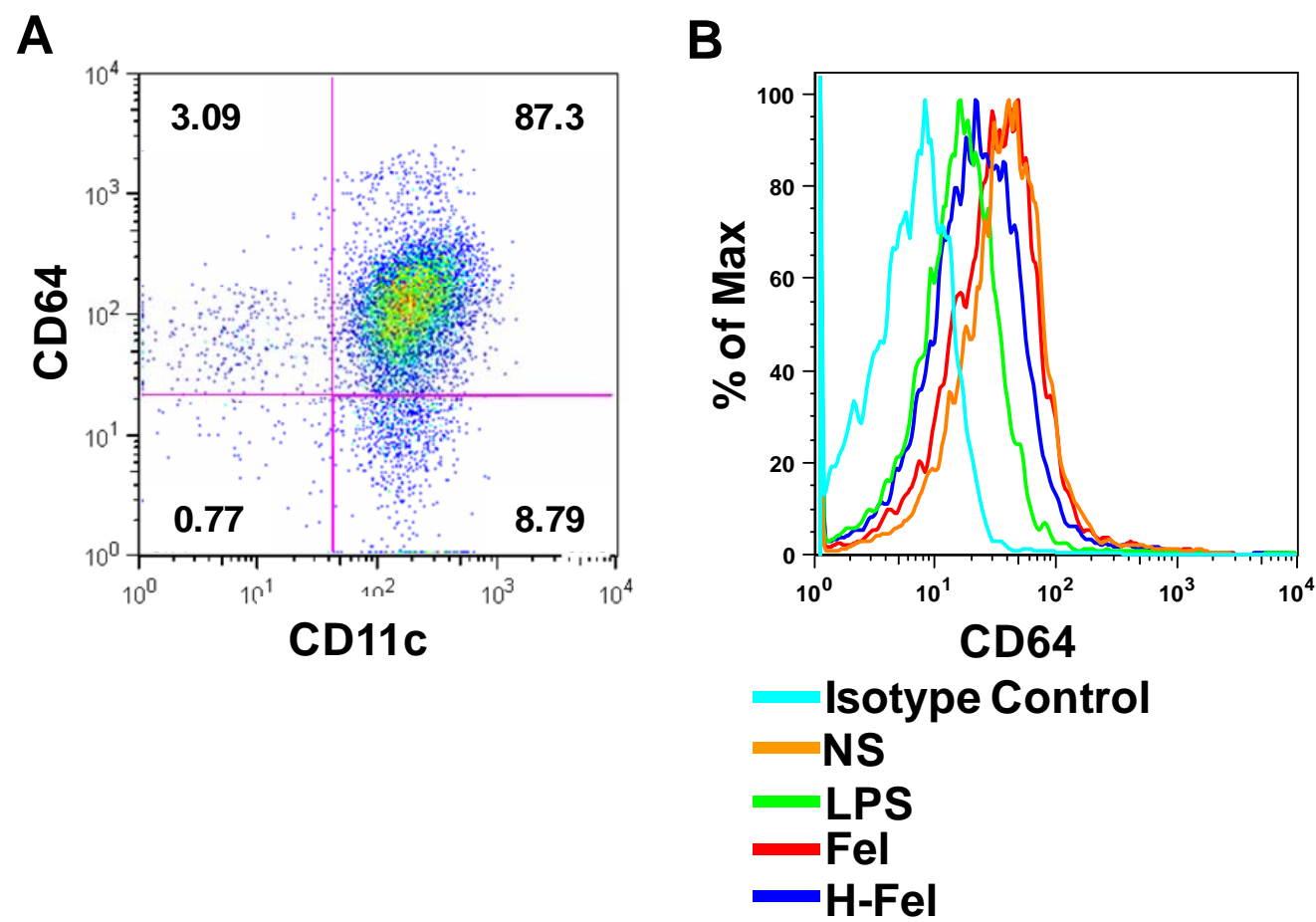
Figure 2.3 H22-Fel d 1 Does Not Alter Expression of Co-Stimulatory Molecules.

MoDCs from subjects with cat allergy $(n=6)$ were pulsed with antigen $(24$ hours) and analyzed for expression of surface antigens by flow cytometry. Effects of different stimuli on maturation markers and CD64. ${ }^{*} p<0.01$. Values represent the means + SDs 


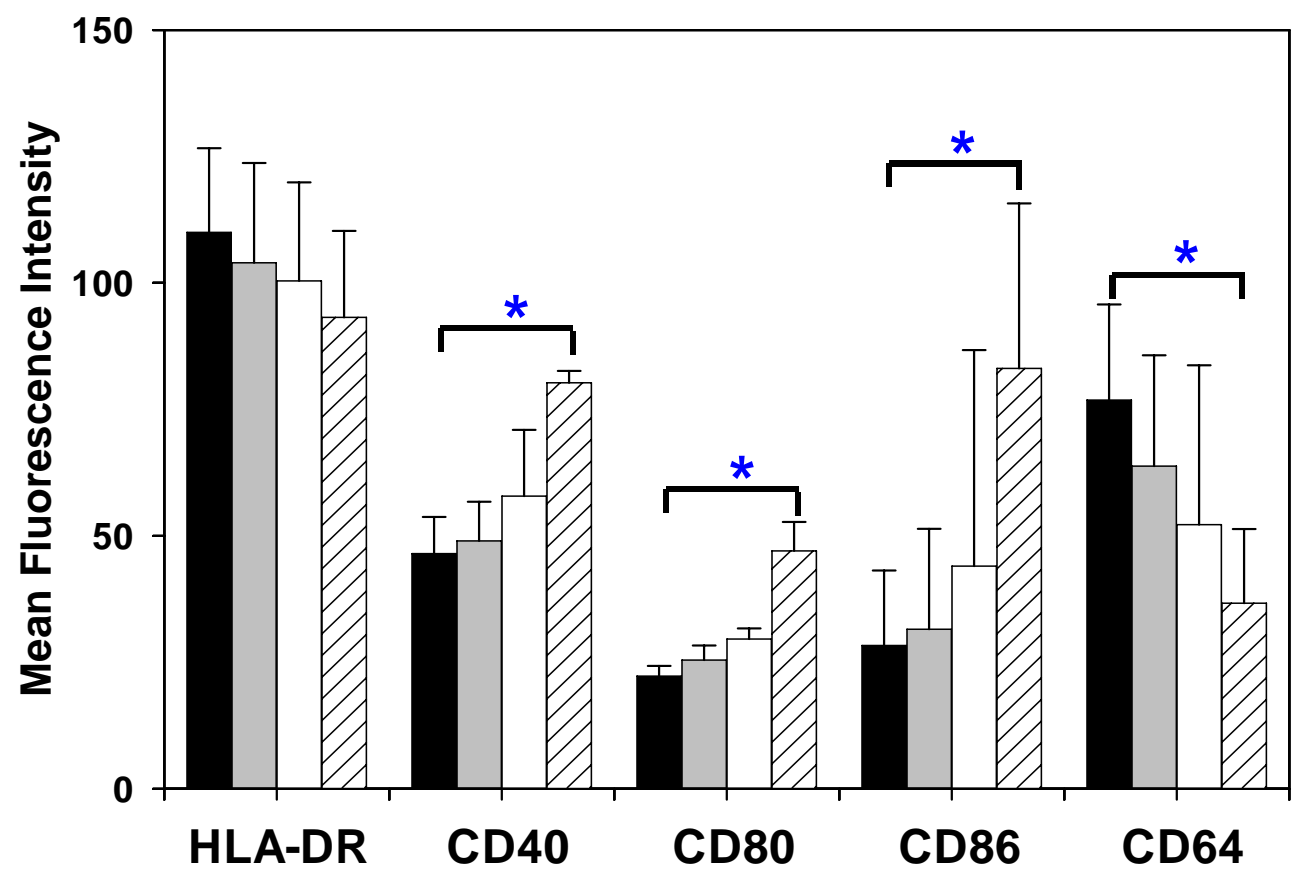

口S $\square$ Fel $\square$ H-Fel 囚LPS 
Figure 2.4 H22-Fel d 1 Enhances Production of Th1-Associated Cytokines and IL-10.

MoDCs from subjects with cat allergy $(n=6)$ were pulsed with antigen $(24$ hours) and analyzed for cytokine secretion by cytometric bead assay. Effects of different stimuli on cytokine secretion. Values represent the mean $\mathrm{pg} / \mathrm{ml}+\mathrm{SDs}$. ${ }^{*} p<005 ;{ }^{* *} p<0.01$ 

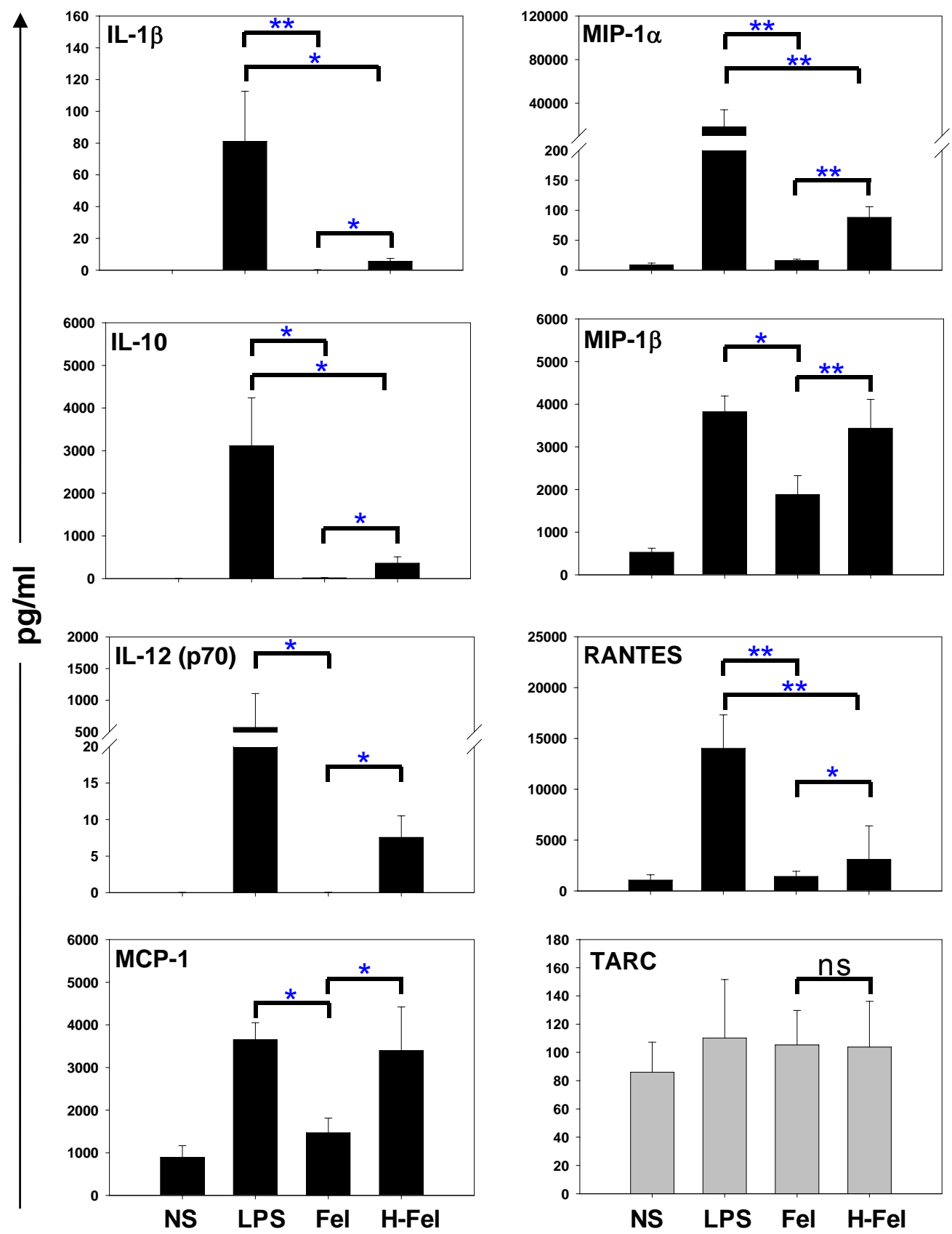
H22-Fel $d 1$ induces increased proliferation in PBMC cultures

Targeting antigens to the high-affinity IgG receptor, FcyRI (CD64), using the $\mathrm{mAb} \mathrm{H} 22$ has been reported to enhance T-cell proliferative responses in vitro (102). We compared proliferation to receptor-targeted Fel d 1 (H22-Fel d 1) with Fel d 1 in PBMC cultures established from cat-allergic subjects and nonallergic subjects with a modified Th2 response to cat ( $n=7$ per group). Stimulation with $\mathrm{H} 22-$ Fel $\mathrm{d} 1$ resulted in increased proliferation compared with Fel $\mathrm{d} 1$ at a dose of $10 \mu \mathrm{g} / \mathrm{mL}$ in both subject groups $(p<.001)$, and this effect was strongest for cultures from subjects with a modified Th2 response $(p<.01$; Figure 2.5$)$.

H22-Fel $d 1$ amplifies IL-5- and IL-10-expressing CD $4^{+} T$ cells and this effect is restricted to cat-allergic subjects

Next we examined whether changes induced by $\mathrm{H} 22-\mathrm{Fel} d \mathrm{~d}$ at the APC level were reflected at the T-cell level. Single-cell analysis of cytokine-producing $\mathrm{CD}^{+} \mathrm{T}$ cells was performed by using flow cytometry after culture of purified $\mathrm{CD}^{+} \mathrm{T}$ cells with allergen-primed moDCs for 10 days. Experiments were performed in 3 groups of subjects with distinct immune responses to cat allergen: (1) cat allergy, (2) modified Th2, and (3) serum antibody-negative controls (Table 2.1). Our objectives were to evaluate changes in the frequency of cytokinepositive $\mathrm{CD}^{+}{ }^{+} \mathrm{T}$ cells and to assess the relevance of these changes to allergic status. We performed a rigorous multiple comparison statistical test to evaluate these changes to provide strong statistical power. H22-Fel d 1 induced increased IL-10+ and IL-5+ CD4 ${ }^{+} \mathrm{T}$ cells compared with Fel d 1, and this effect 
Figure 2.5 Proliferation of PBMCs is Significantly Increased by

\section{H22-Fel d 1.}

PBMCs were stimulated with allergen and proliferation measured on day 7

( $n=7$ per group) by $\left[{ }^{3} \mathrm{H}\right]$-thymidine incorporation. Values correspond to mean

counts per minute + SDs. ${ }^{*} p<0.01 ;{ }^{* *} p<0.001$. 


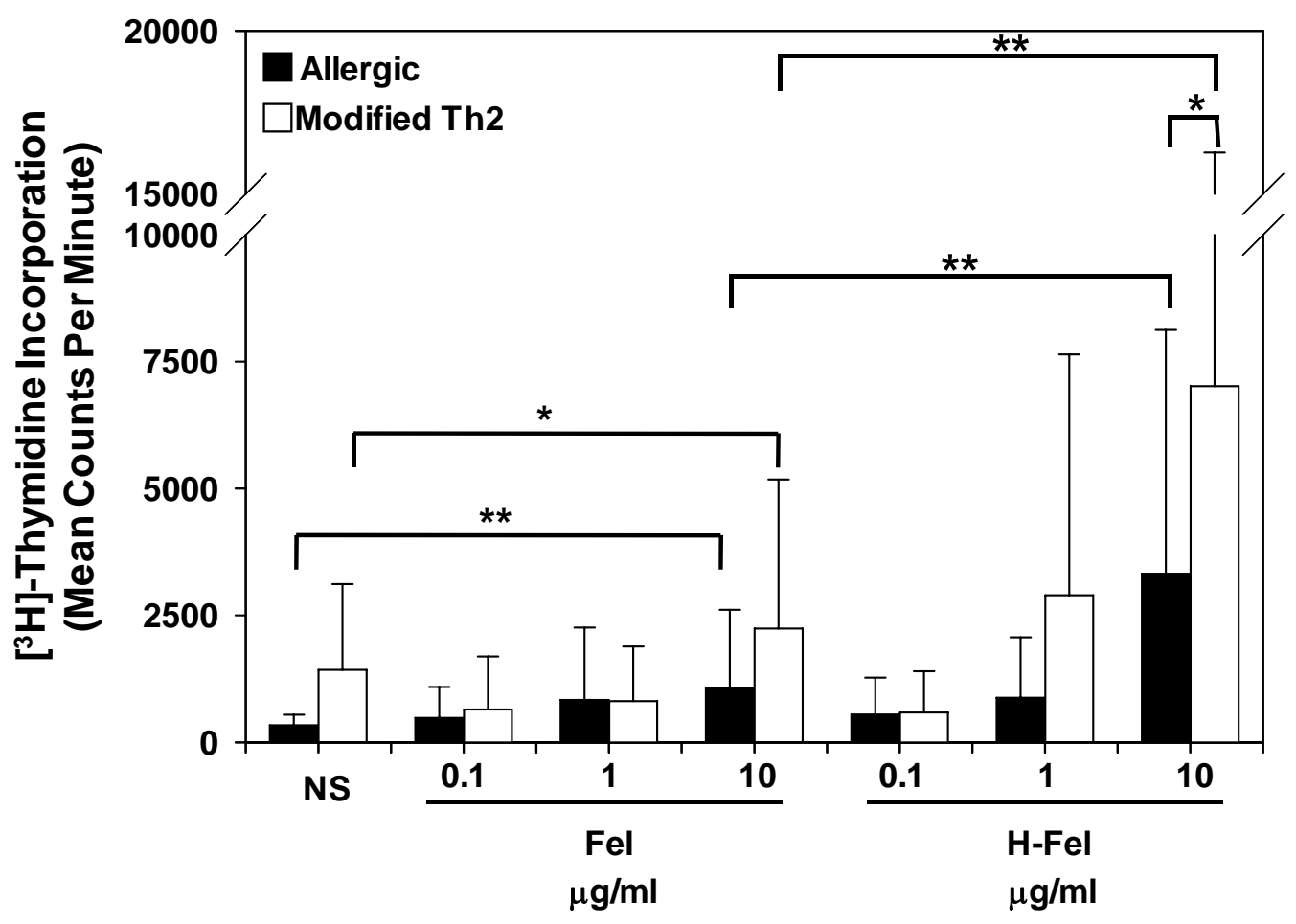


was restricted to subjects with allergy ( $p \leq 0.05$; Figure 2.6). Interestingly, although H22-Fel d 1 induced an increased frequency of IFN- $\gamma+$ cells compared with Fel d 1, this effect was not statistically significant for any group (Figure 2.6).

H22-Fel $d 1$ induces cytokine-secreting subtypes consistent with ThO and regulatory Th1 cells

Single-cell analysis of $\mathrm{CD}^{+} \mathrm{T}$ cells from subjects with allergy revealed that a significant proportion of cytokine-positive cells induced by $\mathrm{H} 22-\mathrm{Fel} \mathrm{d} 1$ expressed more than 1 cytokine (Figure 2.7). To assess further the nature of these cells, we analyzed the following 6 subtypes: (1) IFN- $\gamma+$ only, (2) IL-5+ only, (3) IFN- $\gamma+I L-5+$, (4) IL-10+ only, (5) IL-10+IFN- $\gamma+$, and (6) IL-10+IL-5+. Before adjusting for multiple comparisons, H22-Fel d 1 induced significant increases in all subtypes compared with Fel d $1(p \leq 0.02)$ with the exception of $C D 4^{+} T$ cells expressing IFN- $\gamma$ only $(p=0.25)$. After adjusting for multiple comparisons, changes in 3 subtypes remained significant (IFN- $\gamma+$ IL-5+, IL-10+ only, and IL$10+\mathrm{IFN}-\gamma+$; Figure 2.8 and 2.9). Thus, although $\mathrm{CD} 4^{+} \mathrm{T}$ cells expressing IFN- $\gamma$ alone were not significantly increased, cells expressing IFN- $\gamma$ together with IL-5 or IL-10 were increased, pointing to the production of IFN- $\gamma$ by Th0 $($ IL- $5+$ IFN $-\gamma+)$ and regulatory Th1 (IL-10+IFN $\gamma+$ ) cells. Figure 2.9 is a color graphic summarizing geometric mean data for all cytokine-positive $\mathrm{CD} 4^{+} \mathrm{T}$-cell subtypes in each of the 3 groups of subjects. This figure shows that the T-cell repertoire induced by $\mathrm{H} 22-\mathrm{Fel} \mathrm{d} 1$ was distinct from that induced by Fel $\mathrm{d} 1$ in subjects with a modified Th2 response (compare third bar, upper panel, with second bar, 
Figure 2.6 H22-Fel d 1 Enhances the Frequency of IL-5+ and IL-10+ T Cells.

$\mathrm{CD}^{+} \mathrm{T}$ cells were cocultured with antigen-pulsed moDCs for 10 days, and the percentage of total $\mathrm{CD} 4^{+} \mathrm{T}$ cells that were IFN- $\gamma^{+}, \mathrm{IL}-5+$, and IL-10+ was analyzed by intracellular cytokine staining with flow cytometry. Experiments were performed in subjects with cat allergy $(n=10)$, modified Th2 $(n=5)$, and control $(n=5)$ subjects. Boxes represent medians and interquartile ranges. *Significant after adjusting for multiple comparisons. 


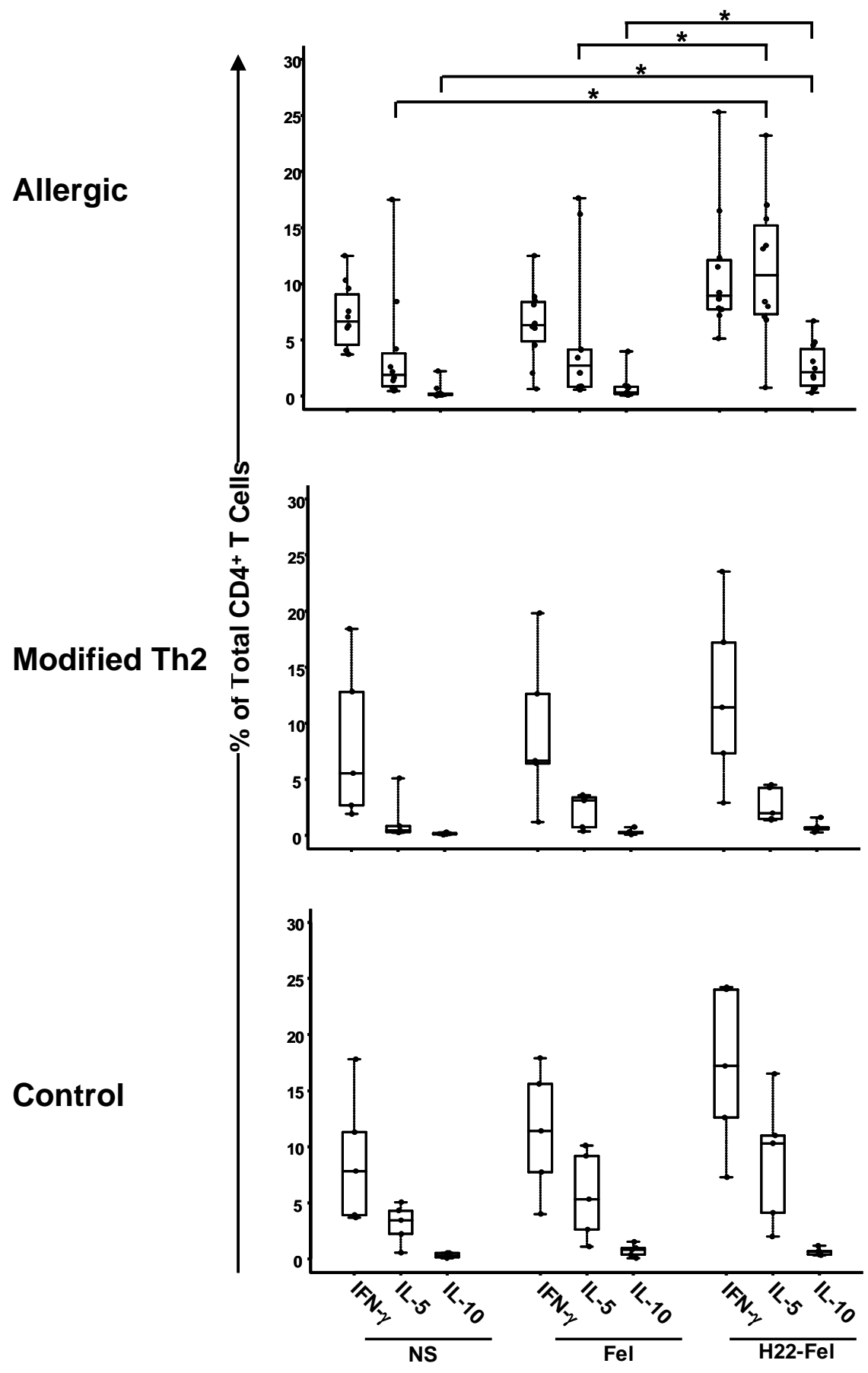


Figure 2.7 Changes in Cytokine-Positive T cells Induced by H22-Fel d 1.

$\mathrm{CD}^{+} \mathrm{T}$ cells were cocultured with antigen-pulsed moDCs for 10 days, and the percentage of total $\mathrm{CD} 4^{+} \mathrm{T}$ cells that were IFN- $\gamma+, \mathrm{IL}-5+$, and IL-10+ was analyzed by intracellular cytokine staining with flow cytometry. Flow cytometry dot plots are shown for 1 of 10 patients with allergy. 


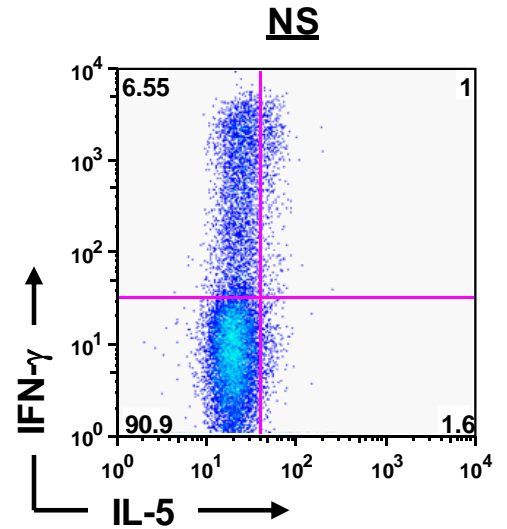

Fel
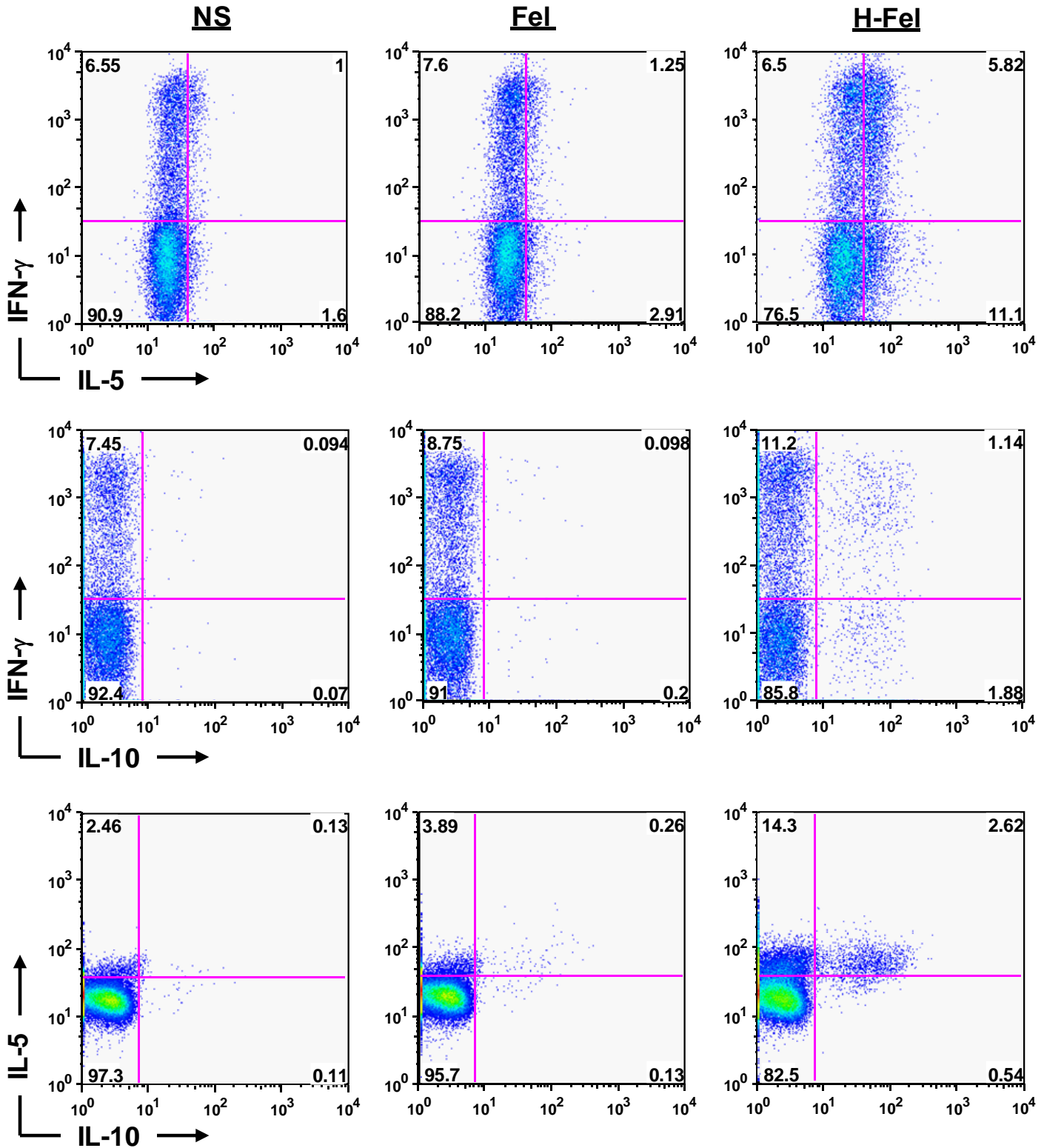
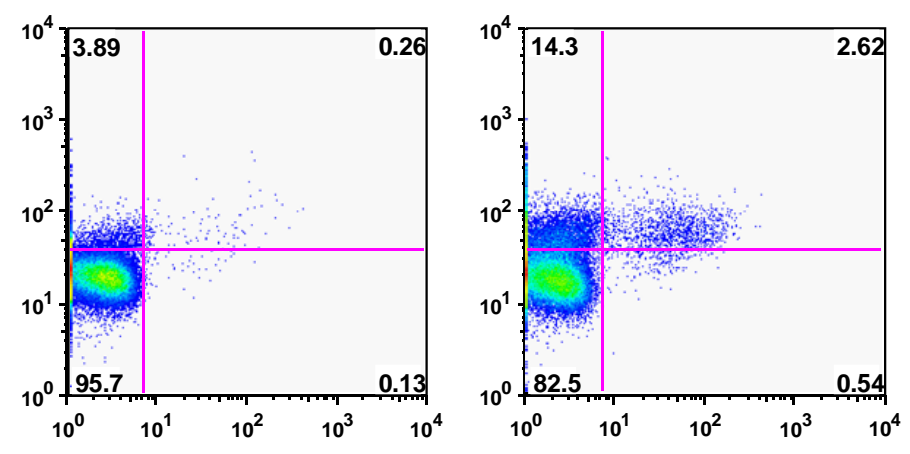
Figure 2.8 H22-Fel d 1 Induces a Qualitative Change in the T Cell Repertoire.

$\mathrm{CD}^{+} \mathrm{T}$ cells were cocultured with moDCs pulsed with Fel d 1 or $\mathrm{H} 22-\mathrm{Fel}$ d 1 for 10 days, and the percentage of the following $6 \mathrm{CD}^{+}{ }^{\mathrm{T}}$-cell subtypes was analyzed by intracellular cytokine staining with flow cytometry: (1) IFN- $\gamma+$ only; (2) IL-5+ only; (3) IFN- $\gamma+$ IL-5+; (4) IL-10+ only; (5) IL-10+IFN- + +; and (6) IL10+IL-5+. Comparison of $6 \mathrm{CD}^{+}$T-cell subtypes induced by Fel d 1 and H22Fel d 1 in subjects with cat allergy $(n=10)$, modified Th2 $(n=5)$, and control $(n=5)$ subjects. Boxes represent median and interquartile ranges of the percentage of total $\mathrm{CD} 4^{+} \mathrm{T}$ cells for each subtype. *Significant after adjusting for multiple comparisons. 


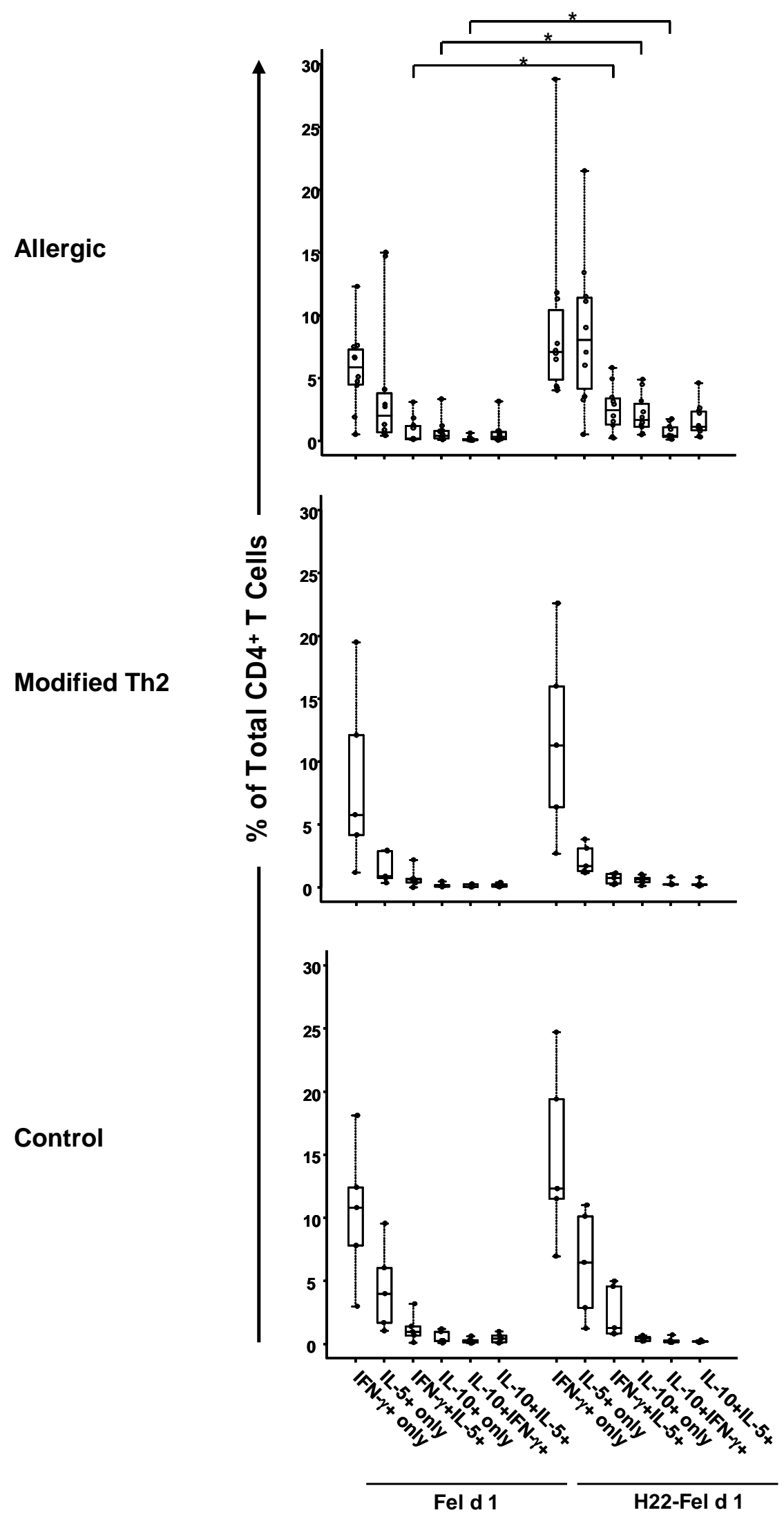


Figure 2.9 Cytokine-Positive T Cell Subtypes Induced by H22-Fel d 1.

$\mathrm{CD}^{+} \mathrm{T}$ cells were cocultured with moDCs pulsed with Fel d 1 or $\mathrm{H} 22-\mathrm{Fel}$ d 1 for 10 days, and the percentage of $6 \mathrm{CD} 4^{+} \mathrm{T}$-cell subtypes was analyzed by intracellular cytokine staining with flow cytometry: Comparison of $6 \mathrm{CD} 4^{+} \mathrm{T}$-cell subtypes induced by Fel d 1 and H22-Fel d 1 in subjects with cat allergy $(n=10)$, modified Th2 $(n=5)$, and control $(n=5)$ subjects. Data represents geometric mean. *Significant after adjusting for multiple comparisons. 

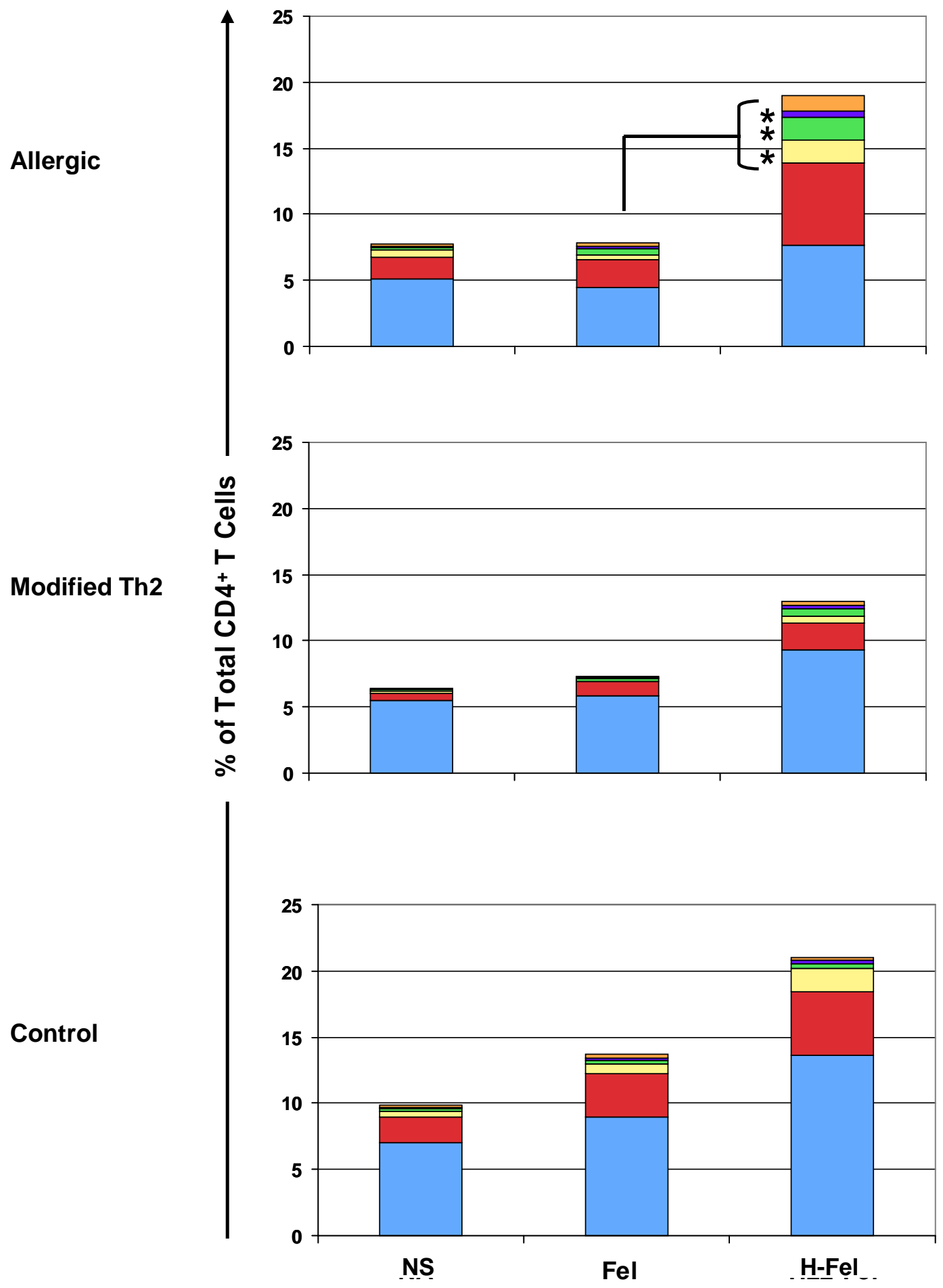

$\square$ IFN- $\gamma+$ only $\square$ IFN- $\gamma+$ IL-5+ $\square$ IL-10+IFN- $\gamma+$ $\square$ IL-5+ only $\square$ IL-10+ only $\square$ IL-10+IL-5+ 
middle panel, Figure 2.9). In summary, H22-Fel d 1 induced a selective increase in Th0 cells and IL-10-expressing T-cell subtypes in CD4 ${ }^{+} \mathrm{T}$ cells from subjects with allergy.

H22-Fel d 1 does not induce a "pro-allergic" repertoire

Given that H22-Fel d 1 stimulated strong T-cell proliferation and induced an increased frequency of IL-5+ CD4 ${ }^{+} \mathrm{T}$ cells (Figures 2.3 and 2.4), we investigated whether the repertoire of cytokine secreting $\mathrm{T}$ cells induced by this molecule was similar to that induced by the prototypic allergen, Der $p$ 1. Five patients were selected for study who were cosensitized to cat and dust mite allergen, and cytokine expression was analyzed in $\mathrm{CD}^{+} \mathrm{T}$ cells after stimulation with H22-Fel d 1 or Der $\mathrm{p} 1$. After adjusting for multiple comparisons, H22-Fel d 1 induced significant increases in Th0 cells (IFN- $\gamma+\mathrm{IL}-5+)$ and all 3 IL-10expressing subtypes (IL-10+ only, IL-10+IFN- $\gamma^{+}$, and IL-10+IL-5+) compared with Der $\mathrm{p} 1$ (Fig 2.10). Thus, the T-cell repertoire induced by H22-Fel d 1 is distinct from a proallergic repertoire, comprising a higher proportion of Th0 cells and diverse IL-10-expressing subtypes.

\section{IL-10 induced by H22-Fel d 1 selectively controls Th2 cells}

Our previous work suggested a role for IL-10 in the development of nonallergic protective responses (modified Th2) to cat allergen (60). Although IL$10+\mathrm{CD}^{+} \mathrm{T}$ cells were increased after stimulation with $\mathrm{H} 22-\mathrm{Fel} \mathrm{d} 1$ in cultures from subjects with allergy, IL-10-expressing T cells represented only a small fraction of total cytokine-positive T cells. This prompted us to question whether 
Figure 2.10 The T Cell Repertoire Induced by H22-Fel d 1 is Distinct from a "Pro-Allergic" Repertoire.

$\mathrm{CD}^{+} \mathrm{T}$ cells were cocultured with moDCs pulsed with Der $\mathrm{p} 1$ or $\mathrm{H} 22-\mathrm{Fel}$ d 1 for 10 days, and the percentage of $6 \mathrm{CD}^{+}$T-cell subtypes was analyzed by intracellular cytokine staining with flow cytometry: Comparison of 6 CD4 ${ }^{+}$T-cell subtypes induced by Der p 1 and H22-Fel d 1 in subjects co-sensitized to dust mite and cat $(n=5)$. A. Boxes represent the median and interquartile ranges of the percentage of total $\mathrm{CD} 4^{+} \mathrm{T}$ cells for each subtype. ${ }^{*}$ Significant after adjusting for multiple comparisons. B. Data from A represented as the geometric mean for each subtype. 
A

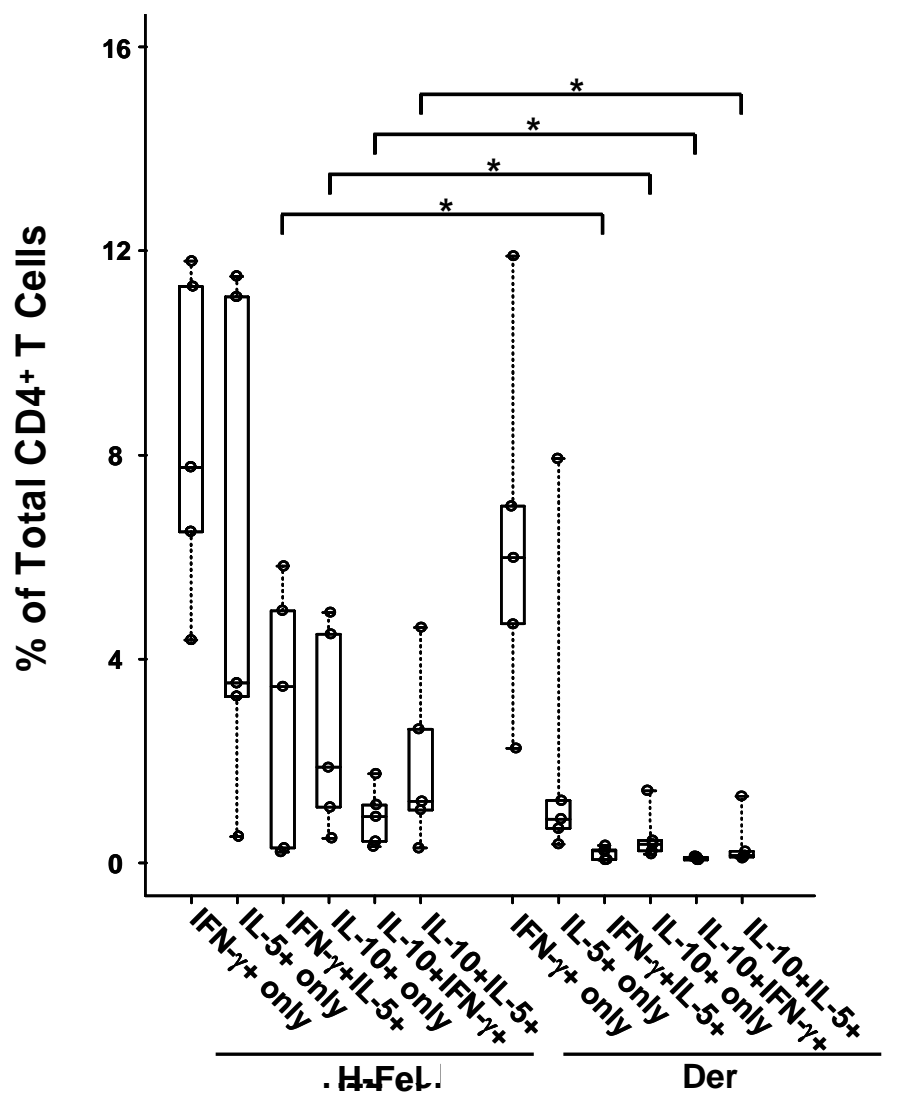

B

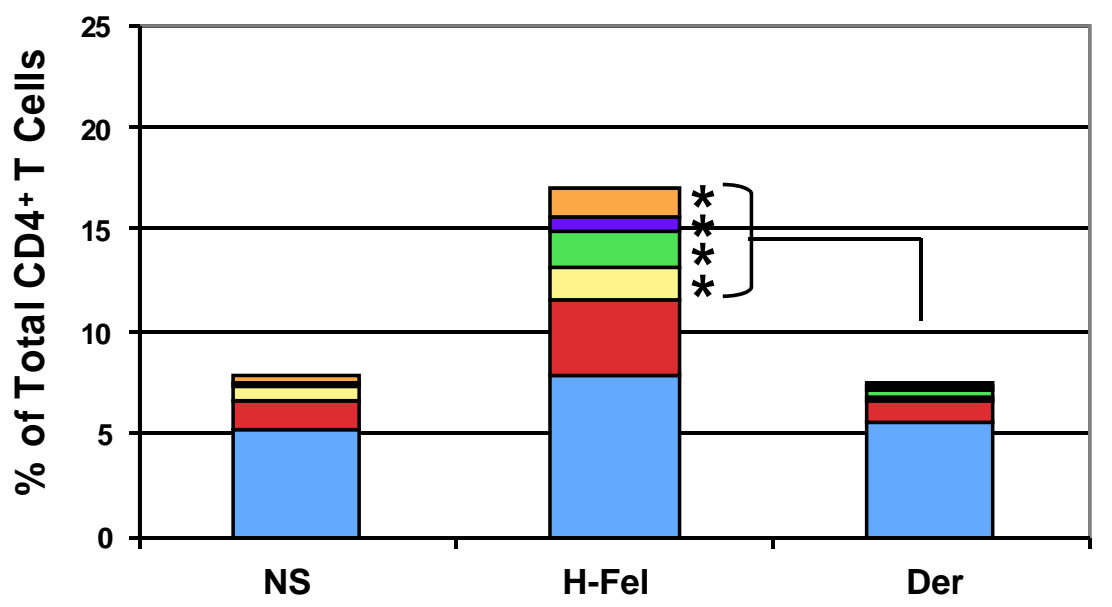

$\square$ IFN- $\gamma+$ only $\square$ IFN- $\gamma+$ IL-5+ $\square$ IL-10+IFN- $\gamma+$ $\square$ IL-5+ only $\square$ IL-10+ only $\square$ IL-10+IL-5+ 
T-cell responses induced by $\mathrm{H} 22-\mathrm{Fel} \mathrm{d} 1$ were controlled. Thus, we examined the effects of IL-10 blockade on the frequency of IL-5+ and IFN- $\gamma+$ CD $4^{+}$T cells. In the presence of antibody to the IL-10 receptor, stimulation with $\mathrm{H} 22-\mathrm{Fel} \mathrm{d} 1$ resulted in an increased frequency of $\mathrm{IL}-5+\mathrm{CD}^{+} \mathrm{T}$ cells in cultures from 4 of 5 subjects with allergy. By contrast, this antibody had little or no effect on IFN- $\gamma+$ or IL-10+ CD4 ${ }^{+} \mathrm{T}$ cells (Figure 2.11). The increase in IL-5+ CD4 ${ }^{+} \mathrm{T}$ cells observed in the presence of anti-IL-10 receptor antibody suggests that the response to $\mathrm{H} 22-\mathrm{Fel} \mathrm{d} 1$ reflects a controlled Th2 response.

\section{Discussion}

Defining the effects of $\mathrm{H} 22-\mathrm{Fel} \mathrm{d} 1$ on the allergic T-cell response may provide a new approach for treatment of patients who have allergy to cats. We have demonstrated that targeting Fel d 1 to $\mathrm{Fc} \gamma \mathrm{RI}$ alters the quality of the CD4 ${ }^{+}$ T-cell repertoire induced by Fel d 1 in subjects with cat allergy. Our findings showed a preferential increase in $\mathrm{CD}^{+} \mathrm{T}$ cells expressing the Th2 cytokine, IL-5, which included Th0 (IL-5+IFN- $\gamma+)$ cells. These changes occurred in concert with increased IL-10-expressing T-cell subtypes. Moreover, we found evidence that IL-5+ CD4 ${ }^{+} \mathrm{T}$ cells induced by H22-Fel d 1 were controlled by IL-10. These observations point to complex changes in the T-cell repertoire at the single-cell level and provide a new variation on existing paradigms related to T-cell modulation.

Other studies have described a fusion protein of Fel d 1 that targets allergen to the inhibitory receptor, FcyRIIb, on mast cells and basophils (138); 
Figure 2.11 IL-10 Blockade Selectively Enhances Th2 Responses.

$\mathrm{CD}^{+} \mathrm{T}$ cells were cocultured with moDCs pulsed with $\mathrm{H} 22-\mathrm{Fel} \mathrm{d} 1$ in the presence of a blocking anti-IL-10 receptor mAb or isotype control $(n=5$ subjects with cat allergy). A. Representative flow cytometry dot plots. B. Frequency of IL$5+$ and IFN- $\gamma+\mathrm{CD}^{+} \mathrm{T}$ cells in the presence or absence of anti-IL-10 receptor (IL10R) mAb for each subject. 
A

H-Fel
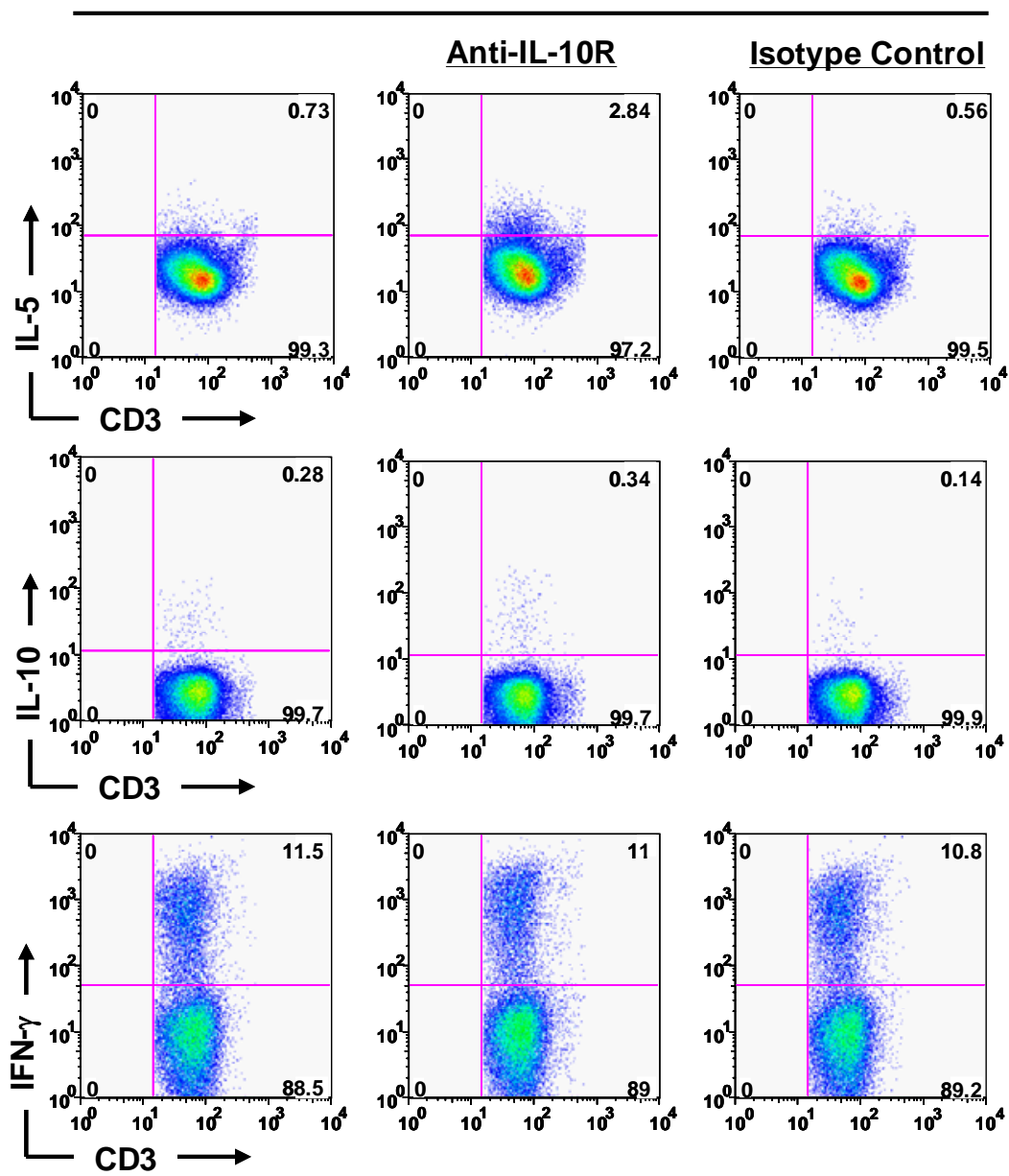

B

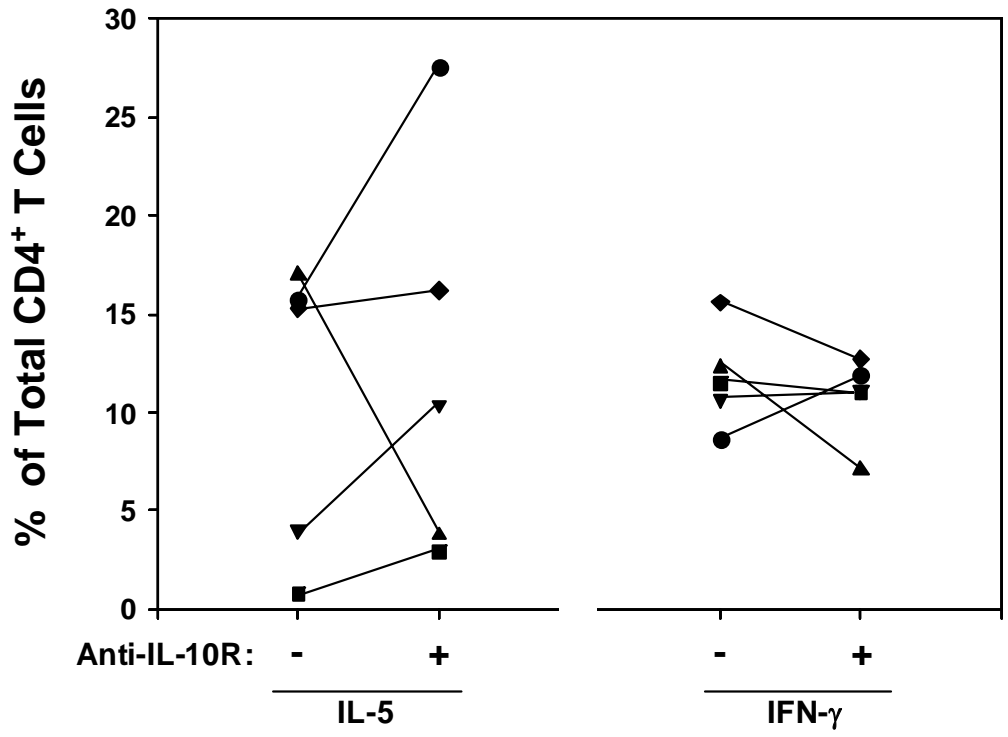


however, the effects of that molecule on T cells were not investigated. In the current study, H22-Fel d 1 selectively enhanced total IL-5+ and IL-10+ CD4 ${ }^{+} \mathrm{T}$ cells, as well as the proportion of these cells coexpressing IFN- $\gamma$. However, no change in $\mathrm{CD}^{+} \mathrm{T}$ cells which secreted IFN- $\gamma$ alone was observed. Thus, H22Fel d 1 induced an altered Th2 response that did not fit with typical Th1 skewing. Furthermore, although IL-5+ $\mathrm{CD}^{+} \mathrm{T}$ cells were a prominent feature of $\mathrm{T}$-cell responses to H22-Fel d 1 in subjects with allergy, these cells were suppressed by IL-10 on the basis of IL-10 blocking experiments. The capacity for H22-Fel d 1 preferentially to increase IL-5+ T cells (and different T-cell subtypes) in cultures from subjects with cat allergy may reflect more efficient antigen uptake and presentation to T cells coupled with a higher precursor frequency of Fel d 1specific T cells in subjects with allergy compared with subjects without allergy (139).

If the T-cell response to H22-Fel d 1 does not signify Th1 skewing per se, then what does it represent? Despite the presence of IL-5+ T cells, the T-cell repertoire induced by this molecule differed from that induced by the prototypic allergen, Der $\mathrm{p} 1$.

Moreover, the repertoire did not resemble the Fel d 1-specific repertoire observed in subjects with a modified Th2 response or subjects without allergy (serum antibody-negative; Figure 2.9). For all comparisons, the prevailing difference was induction of IL-10+CD4 ${ }^{+}$T cells. The population of IL-10expressing cells induced by H22-Fel d 1 included cells that expressed IL-10 alone, or with IFN- $\gamma$ or IL-5. Such coexpression of IFN- $\gamma$ or IL-5 is consistent with 
a regulatory Th1 or regulatory Th2 subtype, respectively. Similar types of IL10-secreting cells have been previously described by Umetsu's group (140, 141). In those studies, induction of regulatory Th1 and Th2 cells was mediated by distinct types of dendritic cells, and both types of regulatory T cells were shown to inhibit the development of Th2-driven airway hyperreactivity and inflammation in mice. Specifically, regulatory Th1 cells were induced by dendritic cells producing both IL-10

and IL-12 (141). In the current study, H22-Fel d 1 induced increased production of IL-10 and IL-12 by dendritic cells from subjects with allergy in conjunction with increased IFN- $\gamma+$ IL-10 + CD4 ${ }^{+}$T cells. Thus, H22-Fel d 1 may condition dendritic cells to prime regulatory Th1 cells in individuals with allergy.

Mature dendritic cells are very efficient at T-cell priming. Such dendritic cells are characterized by high expression of costimulatory molecules (CD40, CD80, and CD86) and production of inflammatory cytokines. Dendritic cells with an immature or semimature phenotype are characterized by lower degrees of expression of costimulatory molecules and/or cytokine production (137). H22-Fel d 1 induced production of inflammatory cytokines by dendritic cells without increased expression of costimulatory molecules, consistent with a semimature phenotype. Such cells are known to exhibit a potent ability to induce regulatory $T$ cells (137). Interestingly, H22-Fel d 1 did not induce increased secretion of Th2promoting cytokines (TARC and eotaxin) by dendritic cells. Thus, these factors are unlikely to promote the generation of IL-5 $+\mathrm{CD} 4^{+} \mathrm{T}$ cells that we observed. Involvement of receptors expressed on dendritic cells (eg, OX40 ligand) provides 
an alternate mechanism and warrants further investigation (119). Taken together, our findings suggest that $\mathrm{H} 22-\mathrm{Fel} \mathrm{d} 1$ induces a dendritic cell phenotype that is intermediate to that induced by Fel $d 1$ and LPS. This semimature phenotype may explain the selective enhancement of IL-5+ CD4 ${ }^{+} \mathrm{T}$ cells, including Th0 cells, in parallel with IL-10-expressing subtypes.

Although H22-Fel d 1 induced enhanced proliferative responses in PBMC cultures from subjects with allergy compared with Fel d 1, proliferation was even higher for cultures from subjects with a modified Th2 response. Despite this, the lowest increase in cytokine-positive T cells was observed for the modified Th2 group. Thus, cytokine production appeared to be dissociated from proliferation. This could arise from altered signaling to T cells mediated at the APC/T-cell interface, which results in uncoupling of distinct signaling pathways within the T cell that are required for cell division and cytokine production (142). Because the modified Th2 response to Fel d 1 may be controlled at the T-cell level (60), it is possible that targeting $\mathrm{H} 22-\mathrm{Fel} d 1$ to $\mathrm{Fc} \gamma \mathrm{RI}$ is not sufficient to overcome those regulatory mechanisms. Interestingly, $<3 \%$ of total cytokine-secreting $\mathrm{CD}^{+} \mathrm{T}$ cells were IL-10+ in cultures from subjects with a modified Th2 response. Thus, low frequencies of IL-10+ T cells may be effective in controlling T-cell responses to allergen.

In summary, we describe a novel variation of a Th2 response to Fel d 1 in subjects with cat allergy. This response, which is induced by targeting Fel $d 1$ to Fc $\gamma \mathrm{RI}$ on dendritic cells, incorporates major elements of a protective T-cell 
response. We propose that targeting allergen to the high-affinity IgG receptor on APCs provides a new approach to T-cell-based vaccines for allergic disease. 


\section{Chapter III - Targeting Allergen to Fc $\gamma$ RI Reveals a Novel Pathway Which Regulates Th2 Responses in Atopic Individuals}

\section{Introduction}

Allergic diseases such as asthma and atopic dermatitis (eczema) are a manifestation of inflammatory processes driven by Th2 lymphocytes. Recently, the IL-7-like cytokine, thymic stromal lymphopoietin (TSLP), has been proposed to act as a major "switch" factor in the allergic response. This premise is based primarily on its capacity to differentiate pro-inflammatory Th2 cells from naïve $\mathrm{CD}^{+} \mathrm{T}$ cell precursors. Such cells secrete high levels of IL-4, IL-5, and IL-13, in conjunction with TNF- $\alpha$, but only low levels of IFN- $\gamma$ and IL-10 $(118,119)$. Induction of these cells has been reported to be mediated by dendritic cells activated by TSLP through the OX40 ligand pathway $(119,143)$. In an extension of these studies, TSLP has been shown to maintain and polarize circulating Th2 central memory cells, including allergen-specific T cells, suggesting an important role for this cytokine in driving Th2 responses associated with established allergic disease (124). More direct evidence of a role for TSLP in the manifestation of distinct allergic diseases is provided in mice which lack the TSLP gene or its receptor. Such animals fail to develop asthma, or else show attenuated disease $(114,116)$. Conversely, mice expressing an inducible TSLP transgene in the skin develop eczematous lesions (115).

In humans, high expression of TSLP is a feature of keratinocytes in the skin lesions of patients with atopic dermatitis (AD) and TSLP is also expressed 
by bronchial epithelial cells derived from the asthmatic lung $(118,119,124$, 144, 145). The interactions of human dendritic cells with TSLP-expressing epithelial cells within the respiratory tract or skin are likely important not only for the generation of Th2 responses associated with allergic disease, but also for recruitment of $T$ cells during ongoing inflammation. For example, human dendritic cells activated by TSLP secrete the chemokine, thymus and activationregulated chemokine (TARC/CCL17). This factor is a key mediator of Th2 cell recruitment to sites of allergic inflammation and TARC is a serum marker of disease severity in $\operatorname{AD}(12,118,119,124,146-154)$. Thus, TSLP may act during both the initiation and the effector phases of the allergic response.

Allergen variants which target the dendritic cell could be a useful approach to treat allergic disease based on their ability to modulate APC function, and thus, alter the quality of allergen-specific $T$ cell responses. Consistent with this view, we recently showed that targeting the major cat allergen, Fel d 1, to the high affinity IgG receptor, $\mathrm{Fc} \gamma \mathrm{RI}$, on dendritic cells using the novel allergen variant, H22-Fel d 1, selectively enhanced the frequency of IL-5- and IL-10expressing T cells in vitro (106). This phenomenon was restricted to T cells isolated from cat-allergic subjects. Interestingly, though the frequency of IL-10expressing $T$ cells was low in our system $(\leq 5 \%$ of total cytokine-expressing cells), IL-10 blockade resulted in a selective enhancement in Th2-like cells induced by $\mathrm{H} 22-\mathrm{Fel} \mathrm{d} 1$, suggesting that $\mathrm{T}$ cell responses were regulated by IL10. Thus, H22-Fel d 1 induced a T cell repertoire which incorporated elements of a protective $\mathrm{T}$ cell response. 
In order to assess whether dendritic cell-based therapies could be efficacious in the clinical setting, it is important to consider the effects of mediators which operate in vivo to modulate APCs in allergic individuals. High expression of TSLP at inflamed sites coupled with the presence of TSLP-primed APCs in regional lymph nodes could subvert the induction of a protective $\mathrm{T}$ cell repertoire to $\mathrm{H} 22-\mathrm{Fel} \mathrm{d} 1$ when this molecule is administered to allergic patients via subcutaneous or sublingual routes, or else by instillation into the respiratory tract. Consistent with this view, we report that Th2 responses to $\mathrm{H} 22-\mathrm{Fel} \mathrm{d} 1$ are amplified in the presence of TSLP and this effect is most marked in atopic subjects. Here, by dissecting the mechanism involved in this process we reveal a novel regulatory pathway linking $\mathrm{Fc} \gamma \mathrm{R}$ signaling to TSLP-driven events. Our findings have important clinical implications for the treatment of Th2-driven inflammatory disorders.

\section{Materials and Methods}

\section{Human Subjects}

Subjects were recruited from the University of Virginia Dermatology Clinic or the University of Virginia Allergic Diseases Clinic. Patients with atopic dermatitis reported physician-diagnosed eczema and the presence of itchy rash was confirmed upon physical exam. All atopic dermatitis patients had moderateto-severe disease based on $\operatorname{SCORAD}$ index $(8,155)$, elevated serum titers of total $\lg E(>250 \mathrm{IU} / \mathrm{ml})$ and high titer $\lg E$ ab to cat extract $(\mathrm{CAP} \geq 0.7 \mathrm{IU} / \mathrm{ml})$. Catallergic subjects without atopic dermatitis were selected based on high titer IgE 
ab to cat extract $(\mathrm{CAP}>0.7 \mathrm{IU} / \mathrm{ml})($ Table 3.1). The presence of Fel d 1-specific $\lg \mathrm{E}$ ab was confirmed in atopic dermatitis patients and cat-allergic patients by Streptavidin CAP assay (132). All atopic dermatitis patients and cat-allergic subjects also had high titer IgE ab to dust mite allergen and were generally sensitized to multiple allergens (Table 3.1). Control subjects had no measurable serum IgG or IgE antibodies to common allergens, including Fel d 1 (Table 3.1). All studies were conducted according to Declaration of Helsinki principles and approved by the University of Virginia Human Investigations Committee.

\section{Cells and Reagents}

Recombinant Allergens: Recombinant Fel d 1 and Fel d 1 targeted to the high affinity IgG receptor, FcyRI, (H22-Fel d 1) were expressed in Pichia pastoris and purified by multi-step chromatography. The purity was $>90 \%$ as determined by SDS-PAGE. The endotoxin content of Fel d 1 and H22-Fel d 1 was comparable (145 EU/ml and $153 \mathrm{EU} / \mathrm{ml}$ respectively).

Cells: MoDCs were generated from CD14 ${ }^{+}$monocytes isolated from fresh PBMCs as described previously (106). These cells were highly homogeneous based on expression of CD64/Fc $\gamma \mathrm{RI}(>90 \%)$ and CD11c (>99\%). Enriched myeloid dendritic cells were isolated from PBMCs by magnetic-activated cell sorting according to manufacturer's instructions (Blood Dendritic Cell Isolation Kit II, Miltenyi Biotec). CD4 ${ }^{+} \mathrm{T}$ cells were purified from fresh PBMCs by negative selection using magnetic-activated cell sorting (Miltenyi Biotec) and purity was 
Table 3.1 Serum Antibody Profiles Associated With Distinct Allergic Phenotypes

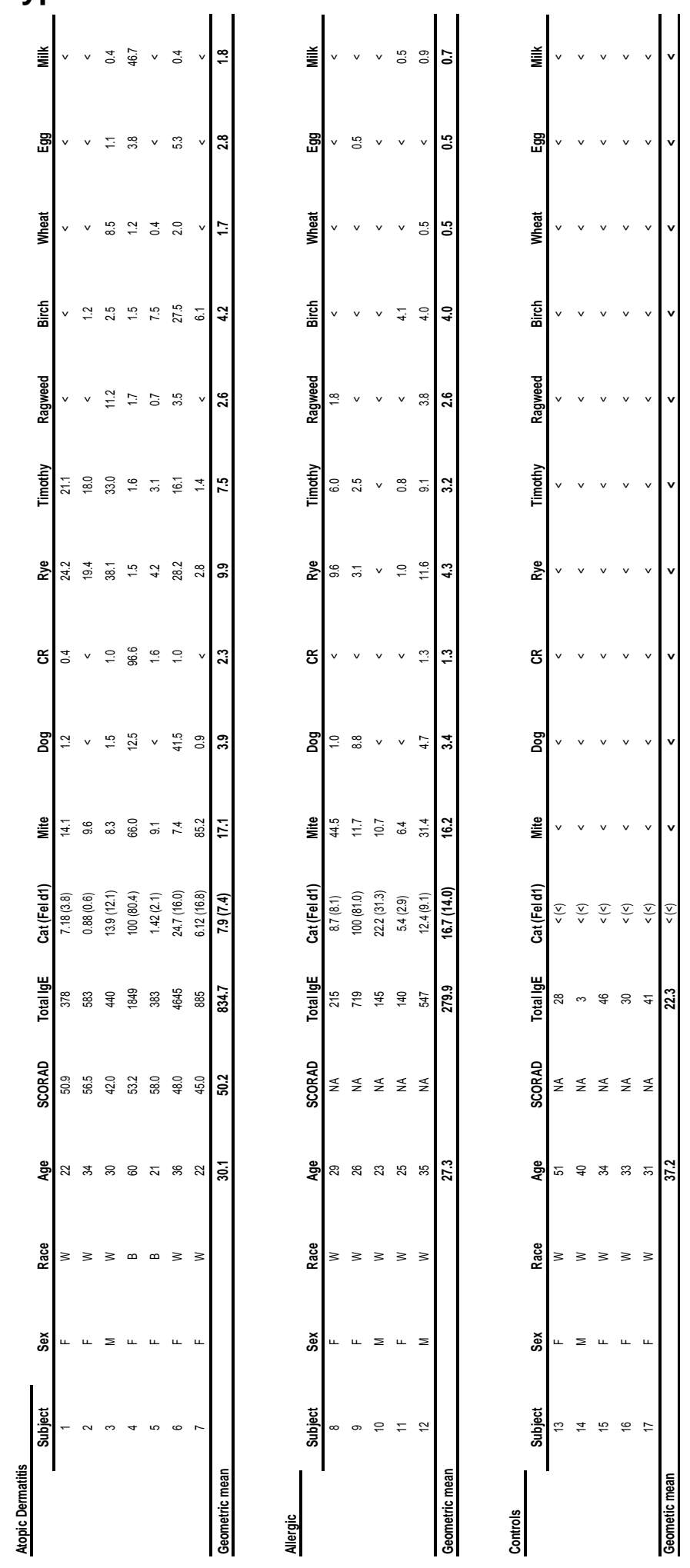


assessed by flow cytometry (>95\%). The THP-1 cell line was generously provided by Kodi Ravichandran.

Flow Cytometry Antibodies and Reagents: Unless otherwise stated, all fluorescently conjugated monoclonal antibodies were purchased from BD Biosciences. Monoclonal antibodies used for surface staining using standard flow cytometry were as follows: PerCP-anti-CD8 (clone SK1); APC-Cy7-anti-CD3 (SK7); APC-anti-TSLP receptor (1B4; Biolegend); PE-anti-OX40 ligand (IK-1); FITC-anti-HLA-DR (L243); FITC-anti-CD40 (5C3); FITC-anti-CD80 (L607.4); and FITC-anti-CD86 (2331). Monoclonal antibodies used for intracellular staining were FITC-conjugated anti-IL-4 (MP4-25D2; R\&D Systems); PE-anti-IL-10 (JES3-9D7); and APC-anti-IFN- $\gamma$ (25723.11). Monoclonal antibodies used for image-based flow cytometry were as follows: PE-anti-TSLP receptor (1A6; EBiosciences); PE-Cy5-anti-CD25 (M-A251); and FITC-anti-CD14 (M5E2). DNA binding dyes used were DRAQ5 (Biostatus Limited) and 7-AAD (BD Biosciences).

Other Antibodies: Anti-OX40 ligand mAb (lk-1) was purchased from R\&D Systems.

Inhibitors: The Syk kinase inhibitor piceatannol, the PI3-kinase inhibitor LY294002, and the protein kinase C inhibitor staurosporine were purchased from Sigma and used at a concentration of $10 \mu \mathrm{M}, 25 \mu \mathrm{M}$ and $1 \mu \mathrm{M}$ respsectively. The Src-related tyrokine kinase inhibitor PP2 was obtained from Calbiochem and used at a concentration of $10 \mu \mathrm{M}$. 
$C D 4^{+} T$ Cell Cultures

MoDCs were pulsed with allergen alone $(10 \mu \mathrm{g} / \mathrm{ml})$, TSLP alone $(15 \mathrm{ng} / \mathrm{ml})$, or both for 48 hours in the presence of complete medium containing $10 \%$ autologous serum as previously described (106). After washing, moDCs were co-cultured $\left(2 \times 10^{5}\right)$ in 24-well plates with autologous CD4 $4^{+}$cells $\left(8 \times 10^{5}\right)$. Cultures were supplemented with recombinant human IL-2 (12U/mI)(BioSource International) on day 5, and then restimulated with PMA $(50 \mathrm{ng} / \mathrm{ml})$ and ionomycin $(2 \mu \mathrm{g} / \mathrm{ml})($ Sigma-Aldrich) for 5 hours on day 10. Brefeldin A (BD Biosciences) was added during the final 4 hours of culture. Cells were stained for surface markers and analyzed by flow cytometry or by image-based flow cytometry. Because restimulation with PMA and ionomycin downregulates surface expression of $\mathrm{CD} 4$, cytokine positive $\mathrm{CD} 4^{+} \mathrm{T}$ cells were identified by gating on $\mathrm{CD}^{+}$cells which were $\mathrm{CD} 8^{\text {neg }}$. Secreted cytokines were measured in culture supernatants harvested on day 10. In some experiments, cells were cultured in the presence of anti-OX40 ligand monoclonal antibody $(20 \mu \mathrm{g} / \mathrm{ml})$. For inhibition studies, moDCs were incubated with Ly294002 or staurosporine for 1 hour $\left(37^{\circ} \mathrm{C}\right)$ prior to pulsing with allergen or TSLP (48 hours). MoDCs were then washed and co-cultured with T cells (7 days).

APC Phenotyping

THP-1 cells or moDCs were pulsed for 24 or 48 hours with allergen alone $(10 \mu \mathrm{g} / \mathrm{ml})$, TSLP alone $(15 \mathrm{ng} / \mathrm{ml})$, or both, in medium containing $10 \%$ autologous serum. Cells were then washed, stained for surface and intracellular markers, 
and analyzed by standard flow cytometry or by image-based flow cytometry. In time-course experiments for OX40 ligand and TSLP receptor expression, allergen and TSLP were removed at 48 hours by washing. In some experiments, APCs were incubated for 1 hour $\left(37^{\circ} \mathrm{C}\right)$ with kinase inhibitors prior to stimulation with allergen (24 hours) and then analyzed for TSLP receptor expression.

\section{Flow Cytometry Analysis}

Standard multi-color flow cytometry was performed on a Becton Dickinson FACSCalibur machine equipped with CellQuest software version 5.2 (BD Biosciences) and upgraded with a $635 \mathrm{~nm}$ red diode laser run by Rainbow software version 1.2b3 (Cytek Development, Gordonsville, VA). Data was analyzed using FlowJo version 6.4.1 (Tree Star Inc., Ashland, OR). For all multicolor analyses compensation controls (single stains - one for each fluorochrome) and gating controls (cells stained with all reagents minus one) were included (156). Image-based flow cytometry was performed on an Imagestream100 (Amnis Corporation) using the manufacturer's collection program, IDEAS. Briefly, debris and cell aggregates were excluded and individual cells were identified by gating on cell area and aspect ratio as confirmed by brightfield (BF) microscopy. Identification of $\mathrm{TSLPr}^{+}$cells was based on selection of distinct cell populations above background fluorescence and confirmed visually by the appearance of a circumferential fluorescence signal. 
Cytokine Assays

Cytokines were measured in culture supernatants harvested from DCs (48 hours) by cytometric bead assay (human 27-plex kit; BioRad, Hercules, CA), or by ELISA (Human CCL17/TARC ELISA (R\&D Systems)). Cytokines in day 10 $\mathrm{CD}^{+} \mathrm{T}$ cell cultures were assayed by cytometric bead assay (Th1/Th2 panel; BioRad). Serum TSLP was measured by monoclonal antibody-based ELISA.

Statistical Analysis

Student's $t$ test was used to compare cytokine levels and mean fluorescence intensity levels in dendritic cell cultures. Analyses were performed using SPSS 14.0 software. In order to account for within-group variability in T cell responses, a rigorous analysis was performed. Briefly, the frequency of cytokine-positive $\mathrm{CD} 4^{+} \mathrm{T}$ cell types stimulated under different conditions was compared by log transforming the percentage of cytokine-positive cells for each condition to derive the geometric mean (gm) value. A T cell ratio was then calculated by dividing the gm value for condition $\mathrm{A}$ by the gm value for condition $\mathrm{B}$ and data displayed as the ratio of these gm values (normalized index) with $95 \%$ confidence intervals. The significance of observed changes was analyzed by general linear mixed effects ANOVA models as previously described (106). The Tukey's multiple comparison type I error rate adjustment was used so that the cumulative type I error rate $(\alpha)$ was $\leq 0.1$, based on the conservative nature of the Tukey's adjustment. The models were fit using PROC MIXED in SAS, version 9.1 (SAS Institute Inc, Cary, NC). 


\section{Results}

The Th2-Promoting Effects of TSLP are Increased in Atopic Subjects.

Despite the capacity for TSLP-primed CD11 ${ }^{+}$dendritic cells to induce a robust Th2 response $(118,119,143)$, the effects of TSLP have not been examined using T cells isolated from subjects with distinct allergic phenotypes. Experiments were performed in the following groups; (1) cat-allergic subjects with atopic dermatitis who were sensitized to multiple allergens [highly atopic]; (2) catallergic subjects without atopoic dermatitis who were sensitized to at least 1 other allergen [atopic]; and (3) healthy non-atopic controls (Table 3.1). For T cell studies, a rigorous statistical approach was used to analyze cytokine-positive $\mathrm{CD}^{+} \mathrm{T}$ cells induced by each stimulus in order to account for low frequency events and within-group variability (106). Our results showed that stimulation of T cells with TSLP-primed monocyte-derived dendritic cells (moDCs) preferentially enhanced IL-4+ T cells and this effect was most marked in atopic dermatitis patients (normalized index=3.0 [1.1-8.3] versus 1.9 [0.7-5] for allergics and 1.7 [0.6-4.5] for controls)(Figure 3.1A and B). However, increases in the frequency of IL-4+ T cells were not significant for any group after adjusting for multiple comparisons. TSLP preferentially induced the secretion of Th2 cytokines with highest levels in cat-allergic subjects with and without atopic dermatitis (Figure 3.2). Thus, the Th2-promoting effects of TSLP were modest irrespective of allergic status, but increased in atopic subjects. 
Figure 3.1 The Th2-Promoting Effects of TSLP are Increased in Atopic Subjects.

Enriched $\mathrm{CD}^{+}{ }^{+} \mathrm{T}$ cells were co-cultured with moDCs which had been pulsed for 48 hours with TSLP. Cells were stained for intracellular cytokines (day 10) and analyzed by flow cytometry, gating on $\mathrm{CD} 4^{+} \mathrm{T}$ cells. A. Relative change in cytokine-positive T cell types as compared with non-stimulated cells was expressed as a normalized index value for each cell type within each group of subjects $(n=5)$. Bars represent geometric means $\pm 95 \%$ confidence intervals. B. Representative flow cytometry dot plots showing the phenotype of gated $\mathrm{CD} 4^{+} \mathrm{T}$ cells from unstimulated (NS) and TSLP-stimulated cultures from 3 subjects with distinct allergic phenotypes. Percentages of cells in each quadrant are shown. Data is representative of 5 subjects in each group. 
A
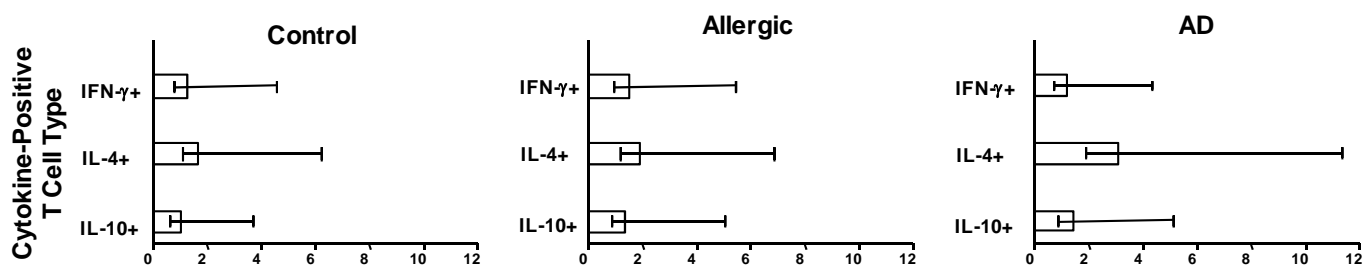

Relative Change (Normalized Index)

\section{B}
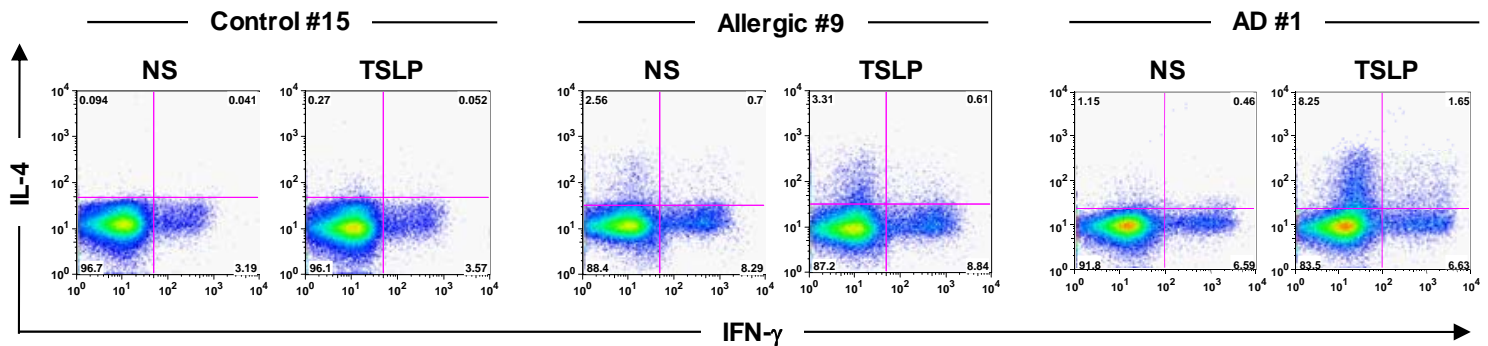
Figure 3.2 Secretion of Th2-Promoting Cytokines is Increased in Atopic Subjects in Response to TSLP.

Enriched $\mathrm{CD}^{+} \mathrm{T}$ cells were co-cultured with moDCs which had been pulsed for 48 hours with TSLP. Levels of secreted cytokines in supernatants harvested from unstimulated (NS) and TSLP-stimulated cultures (day 10) for each group $(n=5)$. Bars represent the mean + SEM. 


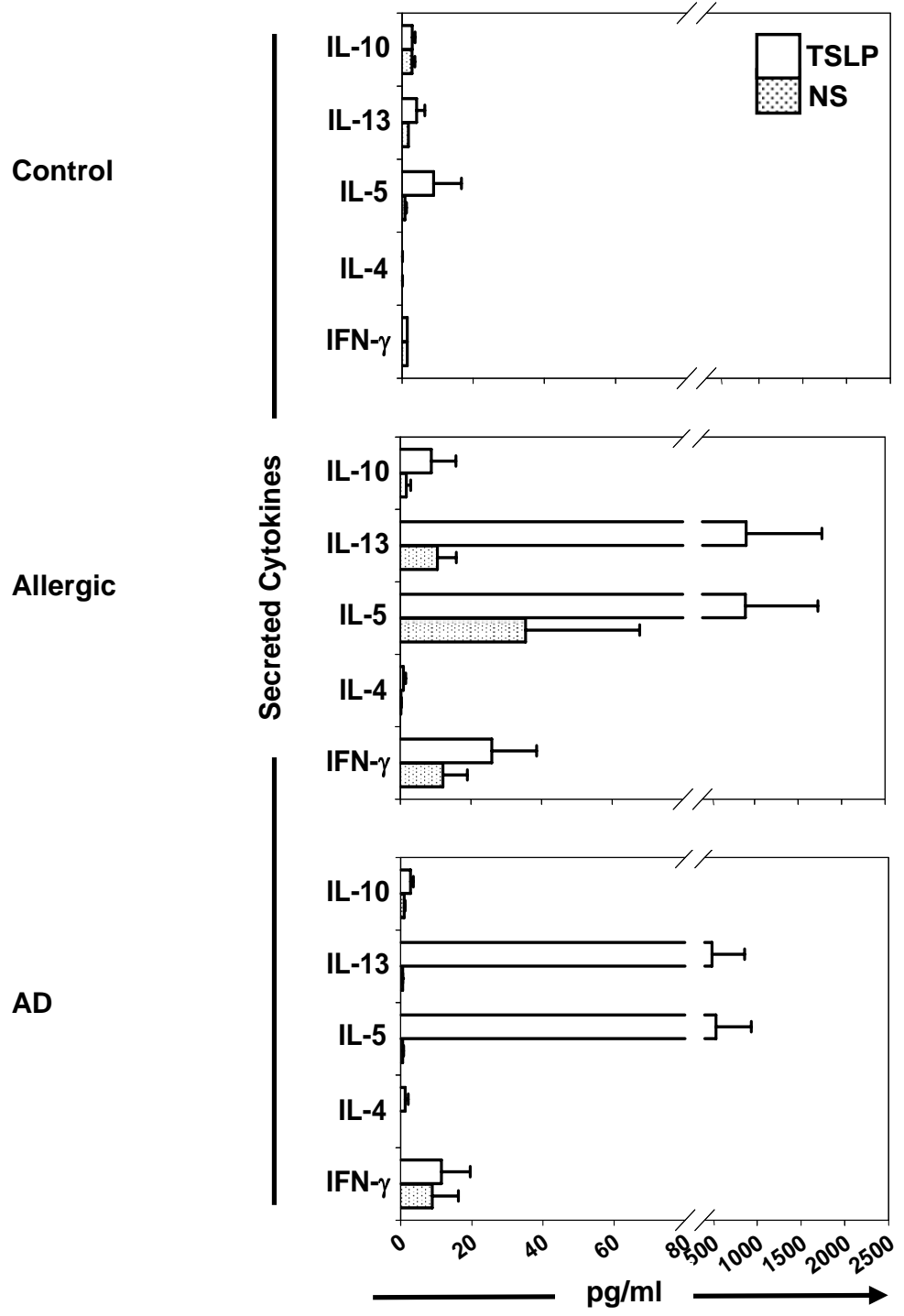


Dendritic Cells Primed with H22-Fel $d 1$ and TSLP Provide a Potent Th2 Stimulus in Atopic Subjects.

Consistent with our previous findings (106), stimulation of T cells with H22-Fel d 1-primed moDCs selectively increased IL-4+ and IL-10+ CD4 ${ }^{+}$T cells in cat-allergic subjects compared with non-receptor-targeted allergen (Fel d 1) $(p<0.05)($ Figure 3.3A and B). We theorized that TSLP would selectively augment Th2 responses induced by H22-Fel d 1 in atopic subjects. However, when moDCs from cat-allergic subjects were primed with $\mathrm{H} 22-\mathrm{Fel} \mathrm{d} 1$ in the presence of TSLP there was no further increase in IL-4+ T cells (Figure 3.3B). Surprisingly, in atopic dermatitis patients, stimulation with $\mathrm{H} 22-\mathrm{Fel} \mathrm{d} 1 \mathrm{did}$ not increase either IL-4+ or IL-10+ T cells as compared with non-receptor-targeted allergen (Figure 3.3B) $(\mathrm{p}>0.1)$. However, when moDCs were primed with $\mathrm{H} 22-\mathrm{Fel}$ d 1+TSLP, IL-4+ T cells were selectively enhanced compared with $\mathrm{H} 22-$ Fel $d 1$ (normalized index $=6.7[2.4-16.7], p<0.05$ ) and this effect was synergistic compared with either stimulus alone (Figures 3.3B and 3.4). Moreover, in contrast to allergics, total cytokine-positive T cells were markedly amplified (Figure 3.3A). Measurement of secreted cytokines showed that moDCs primed with H22-Fel d 1+TSLP selectively enhanced IL-5 and IL-13 in both atopic groups in a synergistic manner (Figure 3.5). Thus, moDCs primed with $\mathrm{H} 22-\mathrm{Fel} \mathrm{d}$ $1+$ TSLP provided a potent Th2 skewing stimulus in cat-allergic subjects and this effect was enhanced in AD patients. Moreover, our findings showed that T cells from atopic dermatitis patients were refractory to the effects of $\mathrm{H} 22-\mathrm{Fel} \mathrm{d} 1$, but this was overcome in the presence of TSLP. 
Figure 3.3 Dendritic Cells Primed with H22-Fel d 1 and TSLP Provide a Potent Th2 Stimulus in Atopic Subjects.

$\mathrm{CD}^{+} \mathrm{T}$ cells were co-cultured with moDCs which had been pulsed for 48 hours with Fel d 1 (Fel), H22-Fel d 1 (H-Fel), TSLP, or H22-Fel d 1+TSLP (HFel+TSLP). Cytokine-positive T cells were analyzed on day 10. A. Summary of cytokine-positive T cell types induced by different stimuli ( 5 subjects per group). Bars represent the geometric mean of the percentage of total CD4 ${ }^{+} \mathrm{T}$ cells for each cell type. *Significant after adjusting for multiple comparisons. B. Relative change in T cell types for (i) H22-Fel d 1 versus Fel d 1, and (ii) H22-Fel d $1+$ TSLP versus H22-Fel d 1 ( $n=5$ per group). *Significant after adjusting for multiple comparisons. 


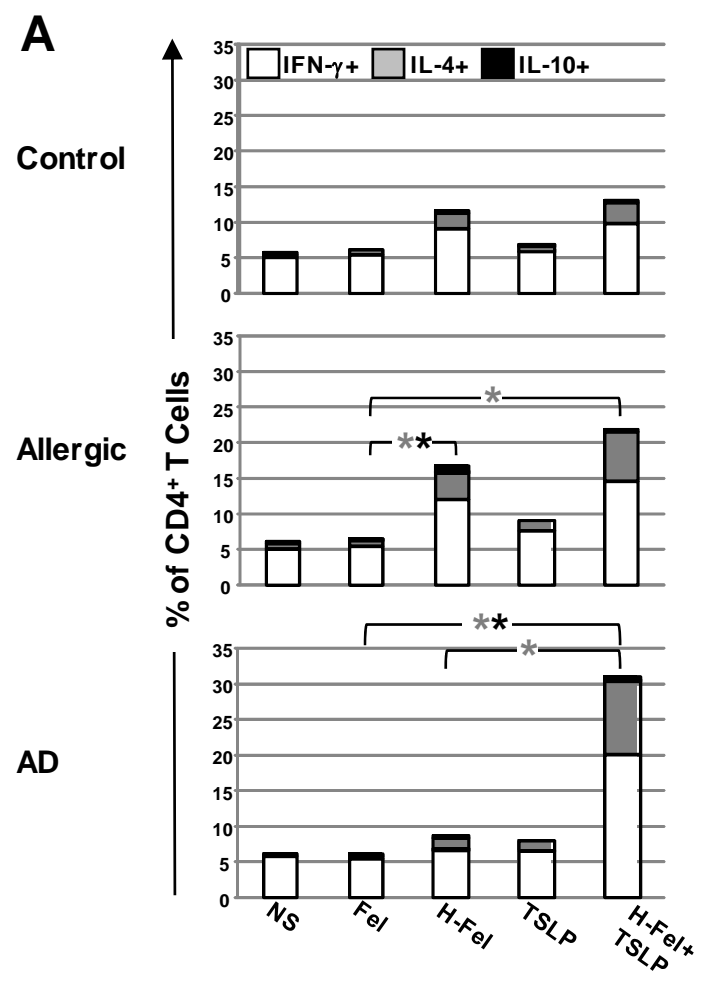

B

(i.) H-Fel vs. Fel

(ii.) H-Fel+TSLP vs. H-Fel

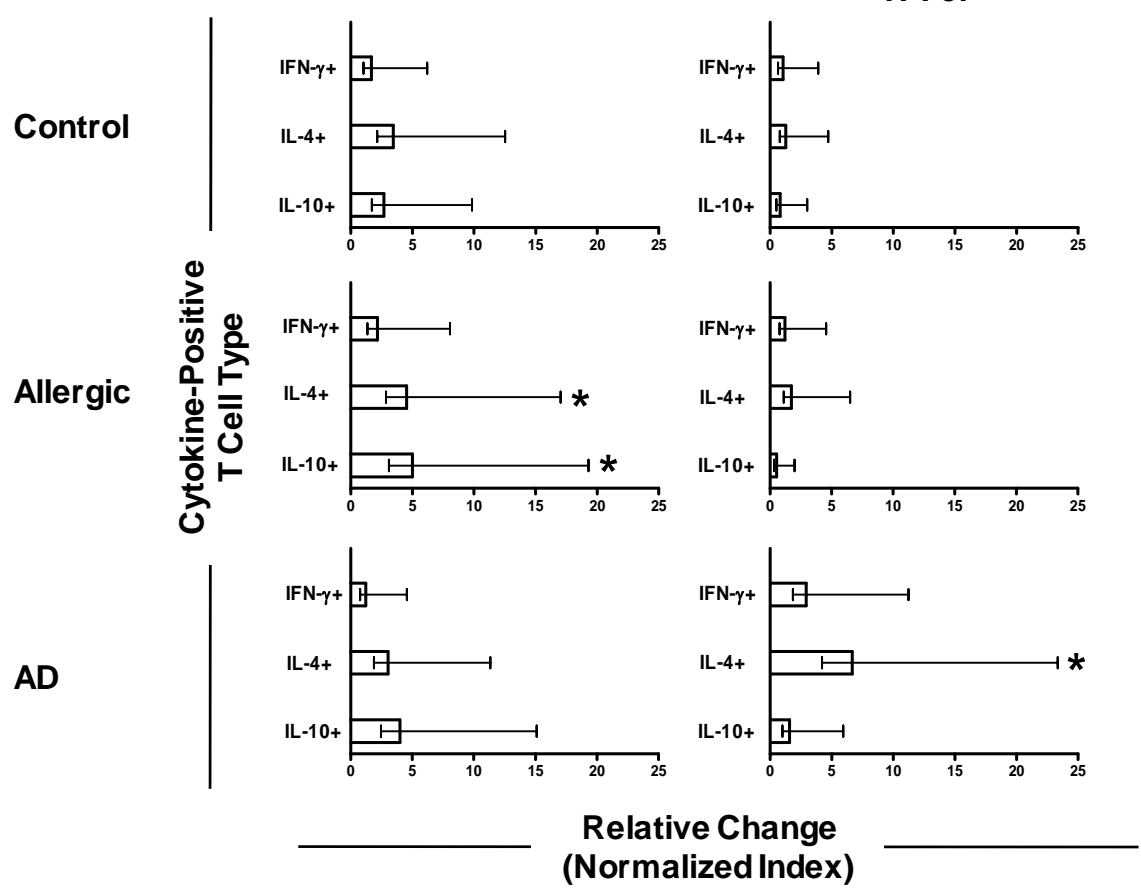


Figure 3.4 Dendritic Cells Primed with H22-Fel d 1 and TSLP Provide a Potent Th2 Stimulus in Atopic Subjects.

$\mathrm{CD}^{+} \mathrm{T}$ cells were co-cultured with moDCs which had been pulsed for 48 hours with Fel d 1 (Fel), H22-Fel d 1 (H-Fel), TSLP, or H22-Fel d 1+TSLP. Cytokine-positive T cells were analyzed on day 10. Representative dot plot from an $\mathrm{AD}$ patient ( 1 of 5 ) showing the phenotype of gated $\mathrm{CD} 4^{+} \mathrm{T}$ cells induced by different stimuli. 


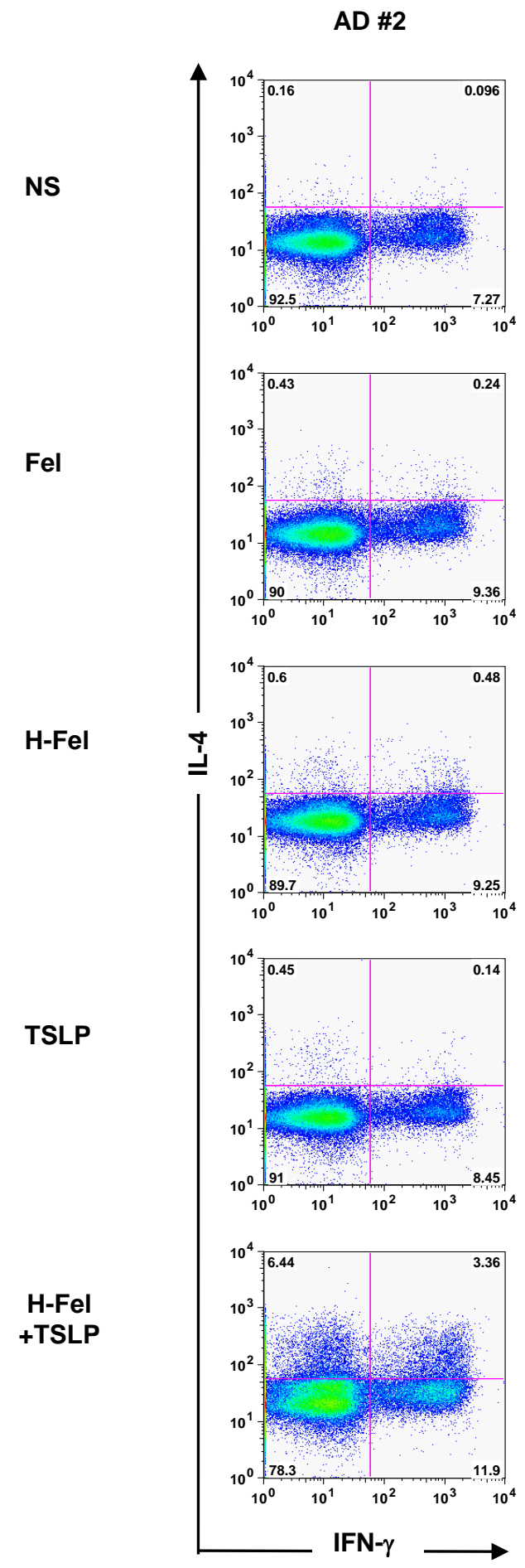


Figure 3.5 T Cells Co-Cultured with Dendritic Cells Primed with H22-Fel d 1 and TSLP Secrete Increased Levels of Th2, but not Th1 Cytokines.

Enriched $\mathrm{CD} 4^{+} \mathrm{T}$ cells were co-cultured with moDCs which had been pulsed for 48 hours with different antigens. Levels of secreted cytokines in supernatants harvested from cultures (day 10) for each group (n=5). Bars represent the mean \pm SEM. 

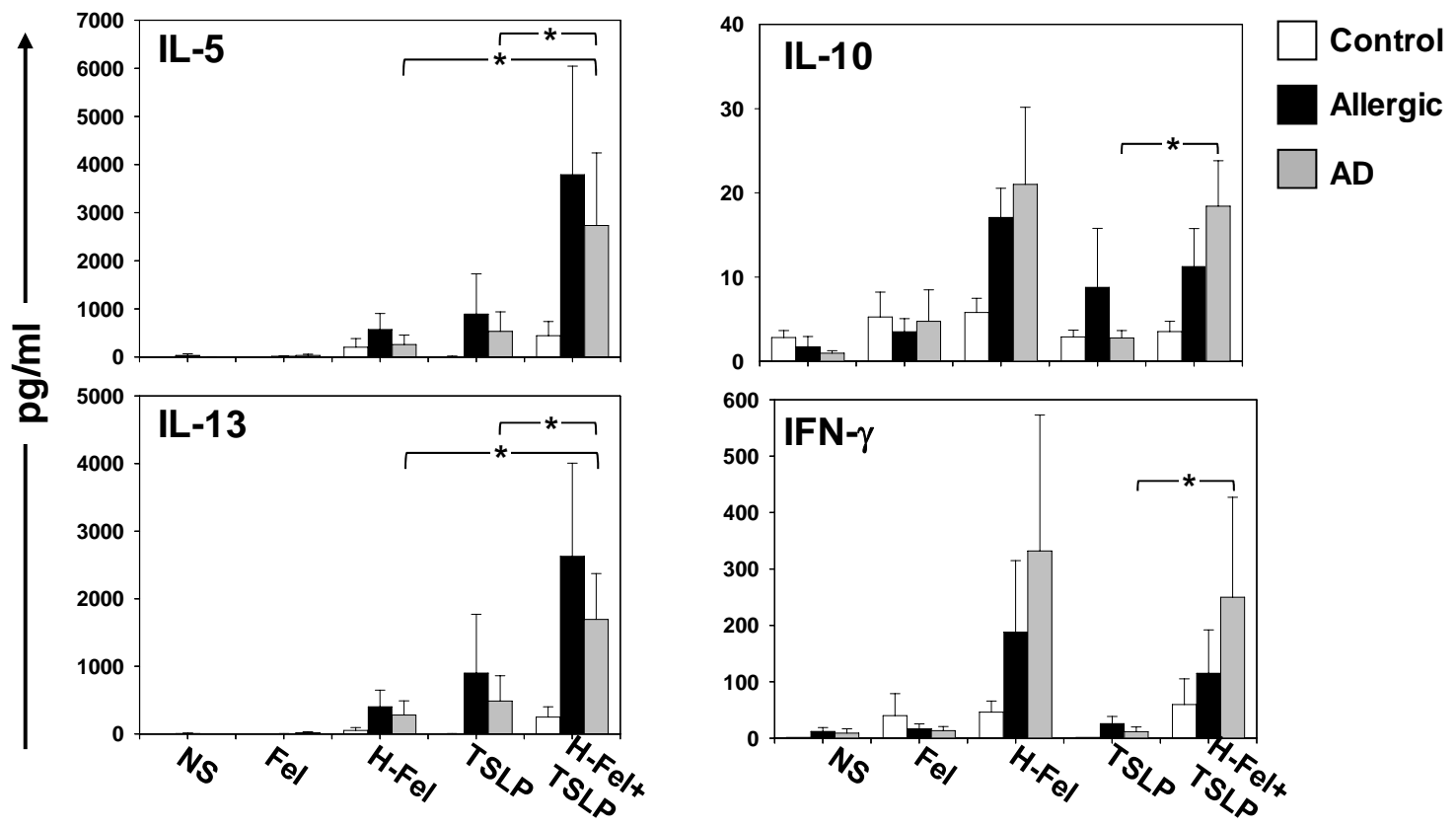
The Effects of TSLP are APC-Mediated

It has recently been reported that TSLP can act directly on activated T cells owing to upregulation of TSLP receptor (TSLPr) after stimulation via the T cell receptor (157). In order to exclude this possibility, TSLP-primed moDCs were washed prior to co-culture with T cells in our system. Nevertheless, cells were cultured in complete medium containing $10 \%$ autologous serum which could, in theory contain trace levels of TSLP. Measurement of TSLP in serum samples isolated from atopic and non-atopic controls using a monoclonal antibody-based ELISA confirmed that levels were very low or undetectable $(<10 \mathrm{pg} / \mathrm{ml})$. Thus, TSLP levels in our culture system were $<1 \mathrm{pg} / \mathrm{ml}$, which is more than 1,000 -fold less than the working concentration of TSLP used by us and other investigators. Thus, we concluded that the effects of TSLP in our system are APC-mediated. Nevertheless, since moDCs primed with H22-Fel d $1+$ TSLP provided a strong T cell activating stimulus, we tested whether this was sufficient to enhance TSLPr expression on T cells. This could provide a mechanism to arm T cells to respond directly to TSLP in vivo. In order to exclude false positives, image-based flow cytometry was performed to visually confirm each $\mathrm{TSLPr}^{+} \mathrm{T}$ cell based on a peripheral zone of staining. By this approach, TSLPr was found to be expressed on $<0.1 \%$ of activated $C D 4^{+} \mathrm{T}$ cells (Figure 3.6). Thus, activation with a potent Th2 stimulus induces only low level expression of TSLPr on T cells. 
Figure 3.6 TSLP Receptor is Expressed on a Small Percentage of CD4 ${ }^{+} \mathrm{T}$ Cells.

Bulk $\mathrm{CD} 4^{+}$were co-cultured with autologous blood dendritic cells and stimulated with allergen + TSLP for 5 days. Cells were stained with surface and intracellular antibodies as indicated and analyzed using the image-based flow cytometry. $\mathrm{CD}^{+}$cells were gated on CD25 and TSLPr. Representative true positive, false positive and true negative cells are shown; arrows indicate location of representative cells. 
True positives

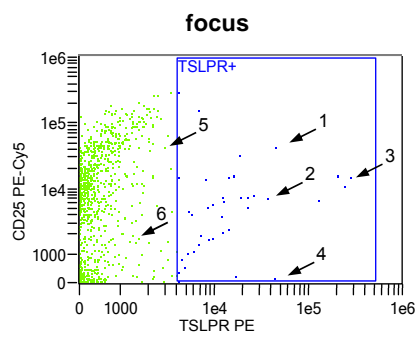

Population Statistics

\begin{tabular}{|l|l|l|}
\hline Population & Count & \%Gated \\
\hline focus \& singlets & 9718 & 100 \\
\hline TSLPR+ \& focus \& singlets & 38 & 0.39 \\
\hline
\end{tabular}

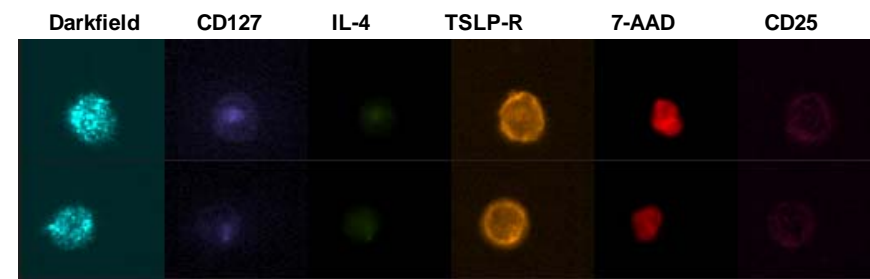

False positives

3

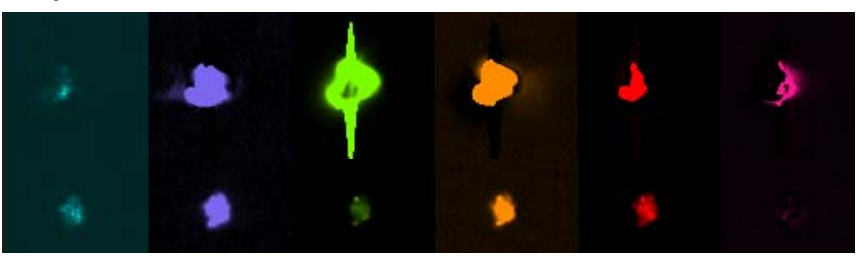

True negatives

5

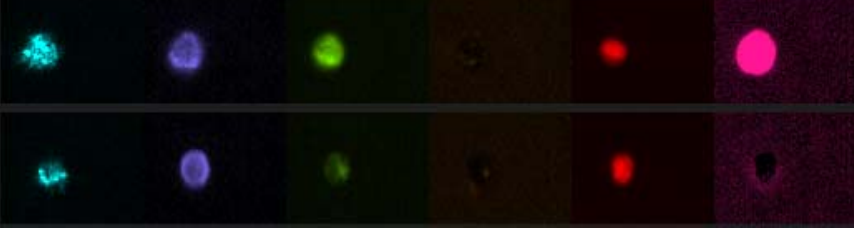


The Th2-Promoting Effect of TSLP on H22-Fel d 1-primed moDCs is OX40 Ligand-Independent.

It was previously reported that TSLP-primed CD11 ${ }^{+}$dendritic cells induced increased expression of OX40 ligand (OX4OL) and that induction of Th2 responses was OX40 ligand-dependent (119). Thus, we investigated whether the Th2-promoting effect of TSLP was mediated via the OX40/OX40L pathway. Surprisingly, TSLP alone did not increase expression of OX40L on moDCs irrespective of allergic status and this was confirmed in time course studies (Figure 3.7A and B). In addition, H22-Fel d 1 had no effect on OX40L expression, though there was a trend towards increased expression of OX40L on H22-Fel d 1-primed moDCs in the presence of TSLP (Figure 3.7A). However, OX40L blockade did not inhibit the induction of IL-4+ T cells by moDCs primed with TSLP alone, H22-Fel d 1 alone, or H22-Fel d 1 + TSLP (Figure 3.8A and B). In order to exclude a role for other factors in the Th2-promoting effects of TSLP, expression of co-stimulatory molecules (HLA-DR, CD40, CD80 and CD86) and a broad array of secreted cytokines (IL-1 $\beta$, IL-1ra, IL-2, IL-4, IL-5, IL-6, IL-7, IL-8, IL-9, IL-10, IL-12 (p70), IL-13, IL-15, IL-17, eotaxin, FGF basic, GM-CSF, IFN- $\gamma$, IP-10, MCP-1, MIP-1 $\alpha$, MIP-1 $\beta$, PDGFbb, RANTES, TNF- $\alpha$, VEGF) was analyzed in moDCs. TSLP did not amplify any of these factors in H22-Fel d 1primed moDCs (Figure 3.9A and B).

It was previously reported that TSLP-primed dendritic cells produced high levels of the Th2-attracting chemokine, CCL17/TARC $(118,119)$. However, TSLP alone did not induce secretion of CCL17 in moDCs and levels of CCL17 
Figure 3.7 The Th2-Promoting Effects of TSLP on H22-Fel d 1-primed moDCs are OX40 Ligand-Independent.

A. MoDCs were pulsed with $\mathrm{H} 22-\mathrm{Fel} \mathrm{d} 1$ (H-Fel), TSLP, or H22-Fel d $1+$ TSLP and expression of OX40 ligand expression was measured by flow cytometry at 48 hours ( $n=5$ per group). B. Representative time course data from one allergic subject. Antigen was washed out after 48 hours. OX40 ligand expression was measured by flow cytometry at the indicated time points. Data is shown for 1 of 2 allergic subjects and 1 of 3 AD subjects. 
A

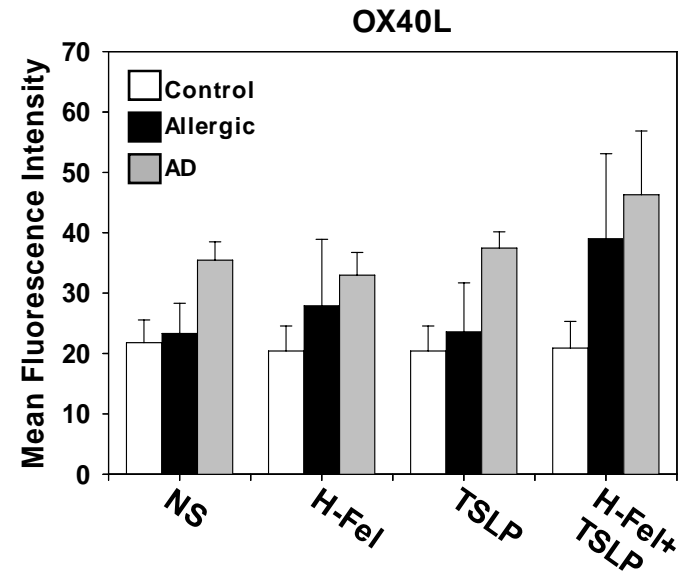

B
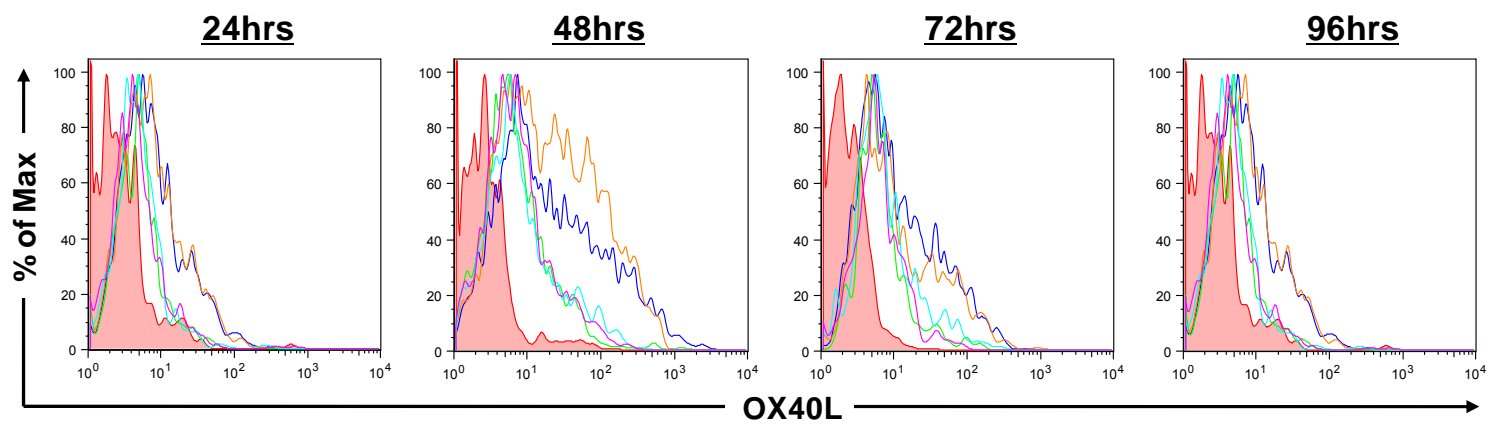

$\square$ NS $\square$ Fel $\square$ H-Fel $\square$ TSLP $\square_{+ \text {TSLP }}^{\text {H-Fel }} \square$ FMo 
Figure 3.8 OX40L Blockade Does Not Inhibit Induction of IL-4+ T Cells.

$\mathrm{CD}^{+} \mathrm{T}$ cells were co-cultured in the presence or absence of anti-OX40L mAb with moDCs which had been pulsed for 48 hours with different stimuli. A. Percentage of IL- $4^{+} T$ cells in the presence or absence of anti-OX40L mAb ( $n=4$ atopic subjects). Bars represent the mean \pm SEM. B. Representative dot plot from an atopic subject (\#8) showing the phenotype of gated $\mathrm{CD} 4^{+} \mathrm{T}$ cells induced by different stimuli. 
A

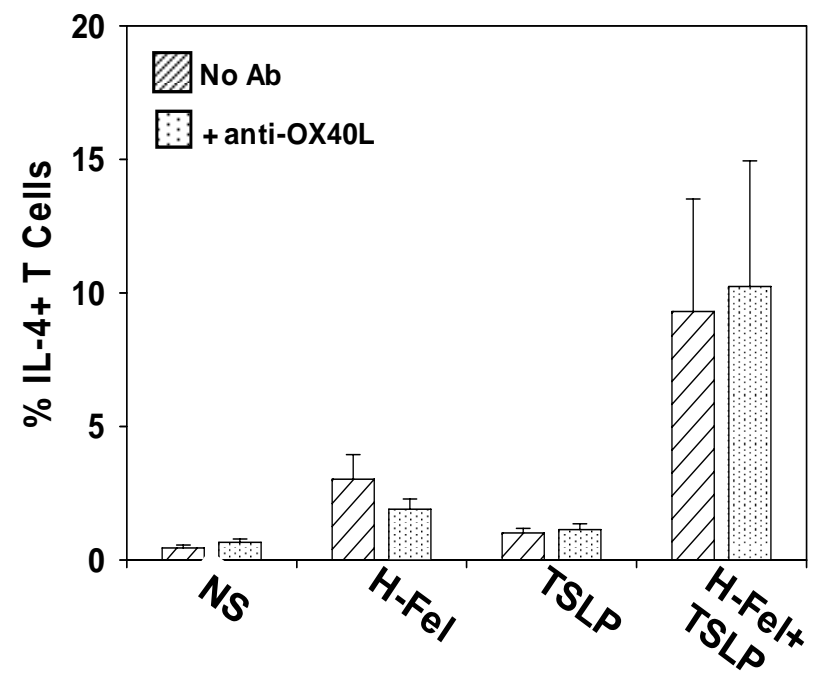

B

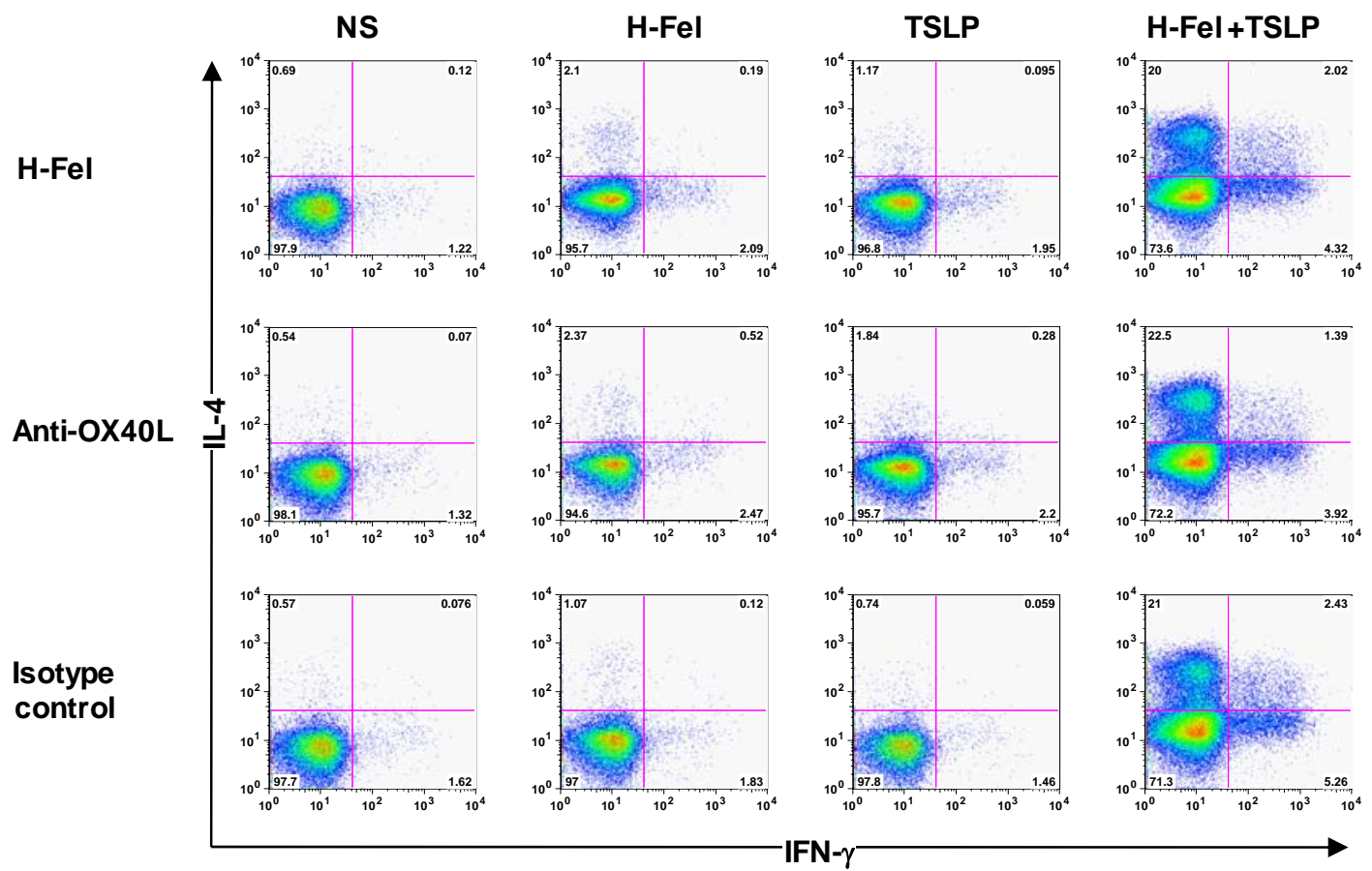


Figure 3.9 TSLP Has No Effect on Co-Stimulatory Molecule Expression or Cytokine Secretion Induced by H22-Fel d 1.

MoDCs were pulsed for $48 \mathrm{hrs}$ with different stimuli. A. Surface expression of co-stimulatory molecules was assessed by flow cytometry. Bars represent mean + SEM ( $n=5$ per group). B. Cytokines were measured from culture supernatants. Representative cytokines shown of 27 analyzed by cytometric bead assay, and TARC was measured by ELISA. Bars represent mean +SEM ( $n=5$ per group). 
A
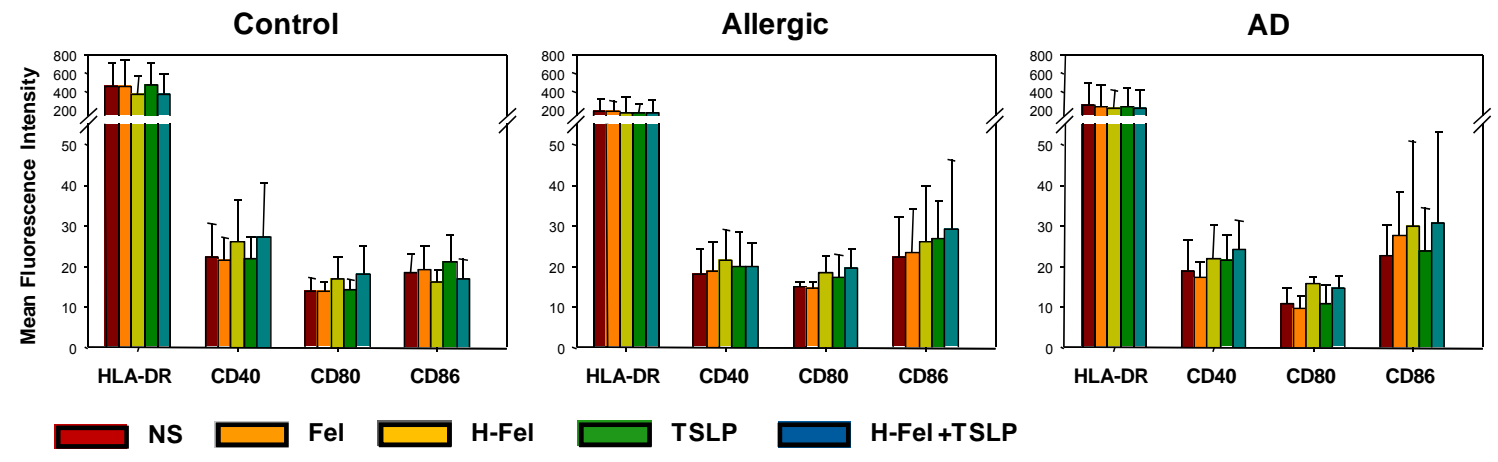

B
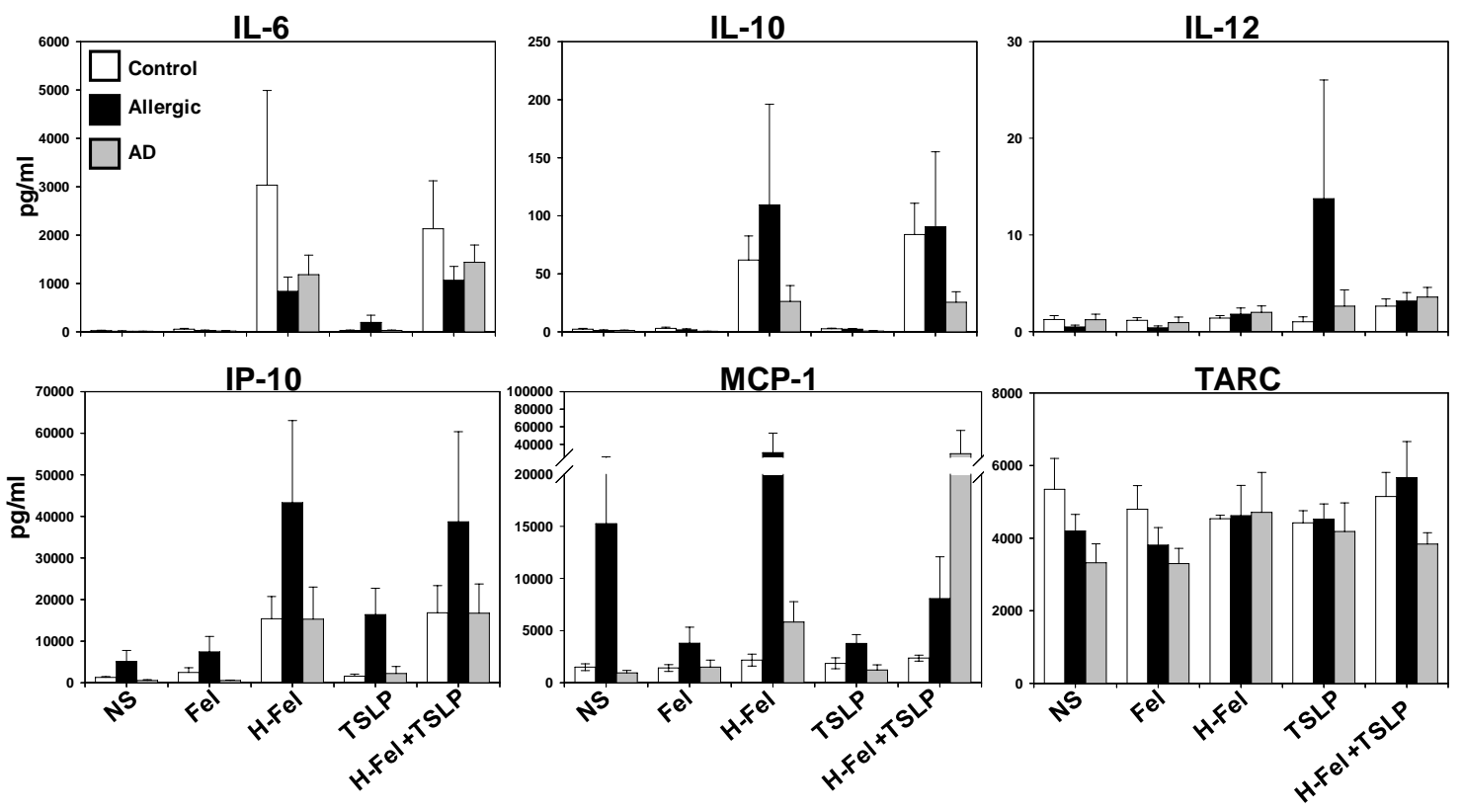
produced by H22-Fel d 1-primed moDCs in the presence of TSLP were comparable to unstimulated cells (Figure 3.9B). Collectively, our findings suggest that the Th2-promoting effects of TSLP on T cells stimulated with $\mathrm{H} 22$ Fel d 1-primed moDCs are mediated through a novel pathway which is OX40Lindependent and not related to TARC production.

H22-Fel d 1 Enhances TSLP Receptor Expression in Atopic MoDCs Through a Pathway Which is Regulated by PI3K and PKC.

The high affinity IgG receptor comprises an $\lg G$ binding $\alpha$-chain complexed with a $\gamma$-chain dimer which contains a signaling motif (immune receptor tyrosine-based activation motif (ITAM)). This motif is critical to an array of Fc $\gamma$ RI-mediated effector functions. Thus, we postulated that targeting allergen to Fc $\gamma$ RI influenced TSLP-mediated pathways in atopic moDCs (ie cells from atopic dermatitis or allergic subjects) through intracellular signaling events. Initially, we examined whether $\mathrm{H} 22-\mathrm{Fel} \mathrm{d} 1$ had the capacity to induce TSLP receptor expression. TSLP receptor was constitutively expressed on atopic moDCs (Figure 3.10). Pulsing with H22-Fel d 1 markedly enhanced TSLPr expression in atopic moDCs with maximal levels expressed at 24 hours (Figure 3.11A). The presence of TSLP had no effect on TSLPr expression at early time points, while at later time points it enhanced TSLPr expression induced by $\mathrm{H} 22-$ Fel d 1 (Figure 3.11A). In contrast to atopic cells, non-atopic moDCs did not constitutively express TSLPr; however, pulsing with H22-Fel d 1 enhanced receptor expression, though to a lesser degree compared with atopic cells 
Figure 3.10 TSLP Receptor is Constitutively Expressed on Atopic MoDCs and Upregulated by H22-Fel d 1.

MoDCs were left unstimulated (NS) or pulsed with Fel d 1 or H22-Fel d 1 before analyzing TSLP receptor expression (24 hours) by flow cytometry. Data is representative of moDCs generated from 4 atopic subjects and 3 non-atopic controls. 


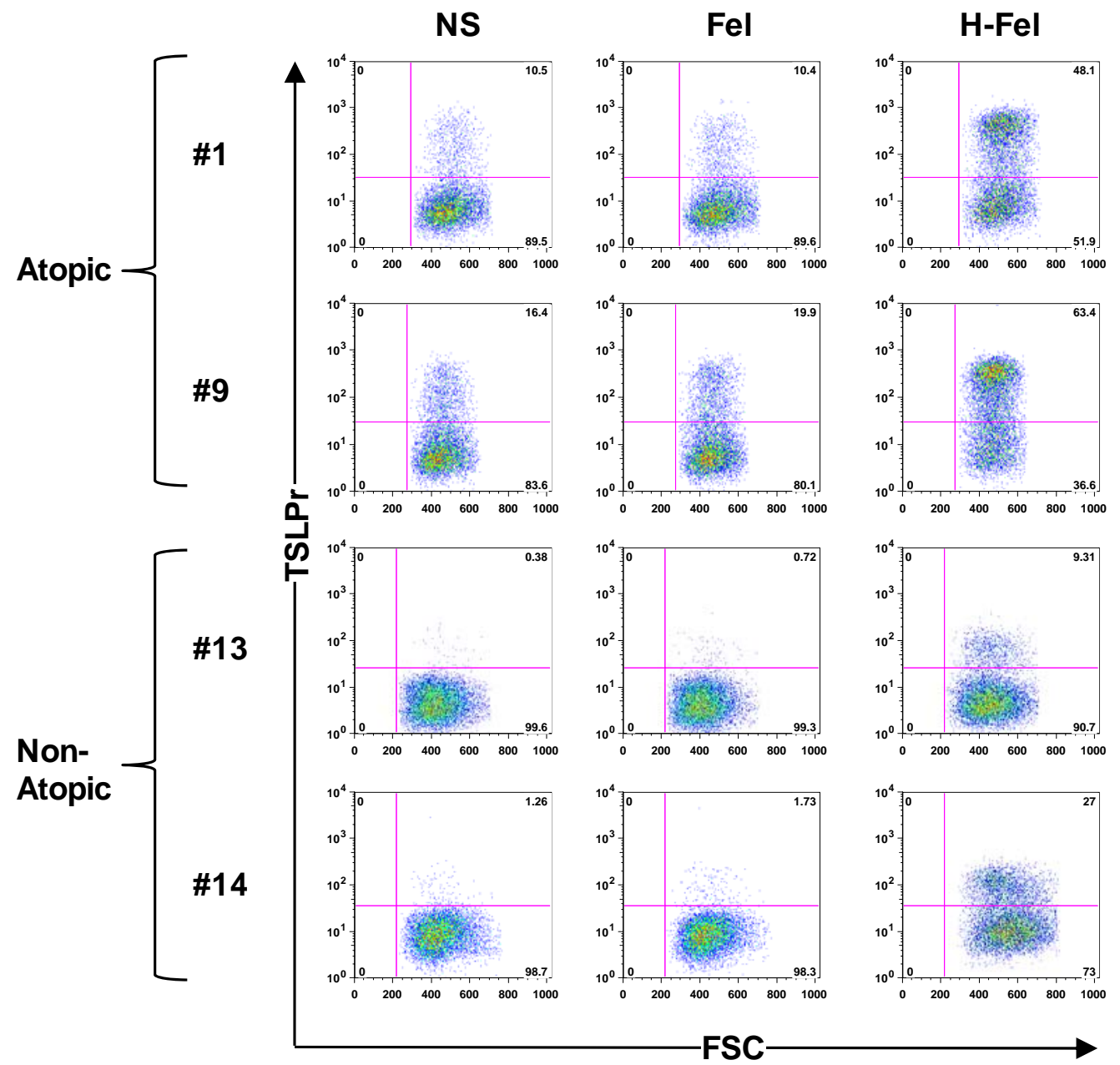


Figure 3.11 H22-Fel d 1-Induced TSLPr Expression Peaks at 24 Hours.

\begin{abstract}
A. MoDCs were primed with the indicated stimuli and TSLP receptor expression was analyzed by flow cytometry. Data is shown for 1 of 3 atopic subjects and 1 of 2 non-atopic subjects. B. Enriched myeloid DCs were isolated from peripheral blood, pulsed with different stimuli, and analyzed at 24 hours for TSLP receptor expression. Data is shown for 1 of 2 atopic subjects.
\end{abstract}


A

moDCs

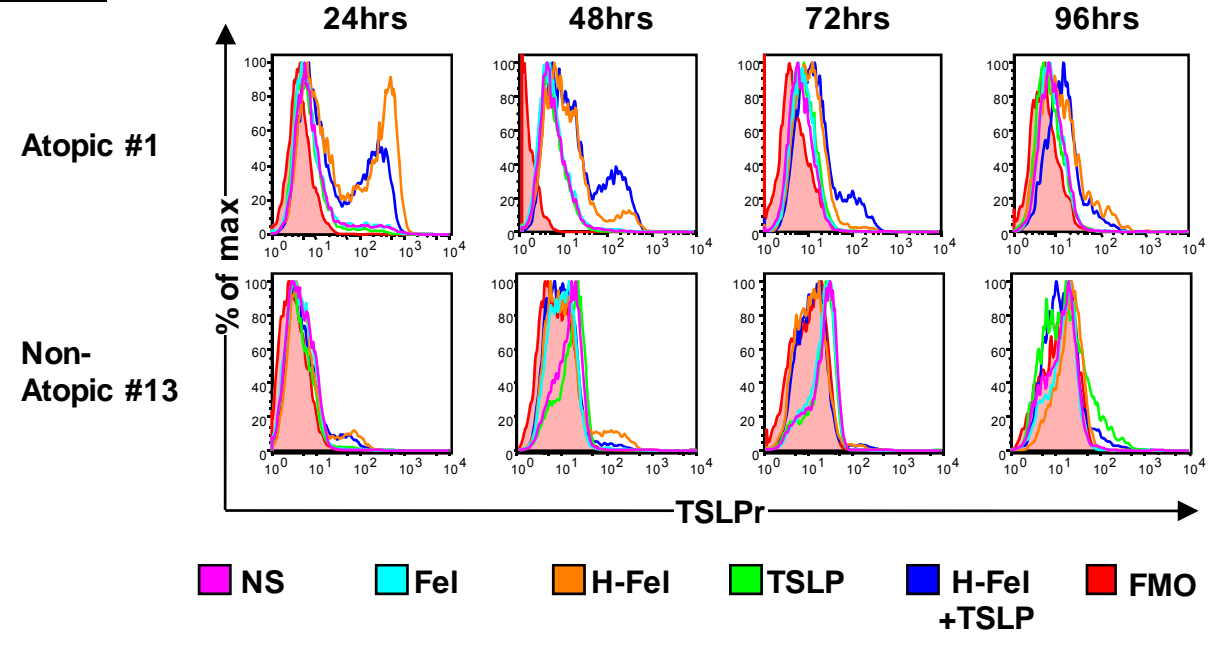

B Myeloid DCs
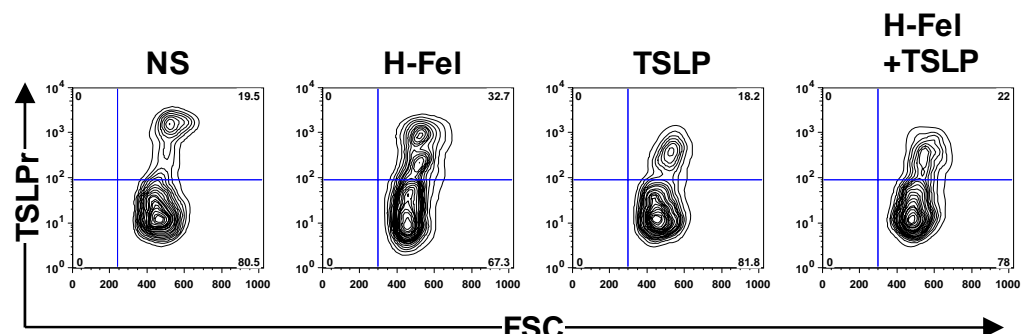

FSC 
(Figures 3.10 and 3.11A). In contrast to H22-Fel d 1, non-receptor-targeted allergen only weakly induced TSLPr expression and this effect was variable and restricted to atopic subjects (Figures 3.10 and $3.11 \mathrm{~A}$ ).

Since the properties of moDCs may differ from myeloid dendritic cells, we next examined whether myeloid dendritic cells freshly isolated from atopic subjects also upregulated TSLPr in response to H22-Fel d 1. Similar to atopic moDCs, cells enriched for myeloid dendritic cells constitutively expressed TSLPr and receptor expression was further enhanced after pulsing with $\mathrm{H} 22-$ Fel $\mathrm{d} 1$ (Figure 3.11B).

The H22 sFv domain of the H22-Fel d 1 molecule binds outside the Fc binding pocket and is monovalent. As such, it does not cross-link Fc $\gamma \mathrm{RI}$. Though cross-linking is not necessarily required for Fc $\gamma \mathrm{RI}$-mediated endocytosis, IgG antibodies are required for internalization of $\mathrm{H} 22 \mathrm{sFv}$ bound to $\mathrm{Fc} \gamma \mathrm{RI}(105,158)$. Thus, we tested whether serum from subjects with distinct allergic phenotypes influenced TSLPr expression. To do this, we used THP-1 monocytes which have been used extensively to characterize cellular events triggered by ligation of Fc $\gamma$ RI. Interestingly, H22-Fel d 1-mediated upregulation of TSLPr was enhanced in serum from $A D$ subjects as compared with fetal bovine serum which does not bind to human Fc receptors; however, TSLPr expression was only modestly increased in atopic dermatitis serum as compared with control serum (Figure 3.12). These findings suggested that, while serum antibodies may facilitate endocytosis, the difference in the capacity to upregulate TSLPr expression between atopic and non-atopic moDCs was primarily intrinsic to these cells. 
Figure 3.12 Serum from Atopic or Non-Atopic Subjects Does Not Affect TSLPr Expression.

THP-1 monocytes were pulsed with H22-Fel d 1 in the presence of FBS or serum from atopic and non-atopic subjects and TSLP receptor was measured by flow cytometry at 24 hours. Data is representative of 8 experiments. 
THP-1

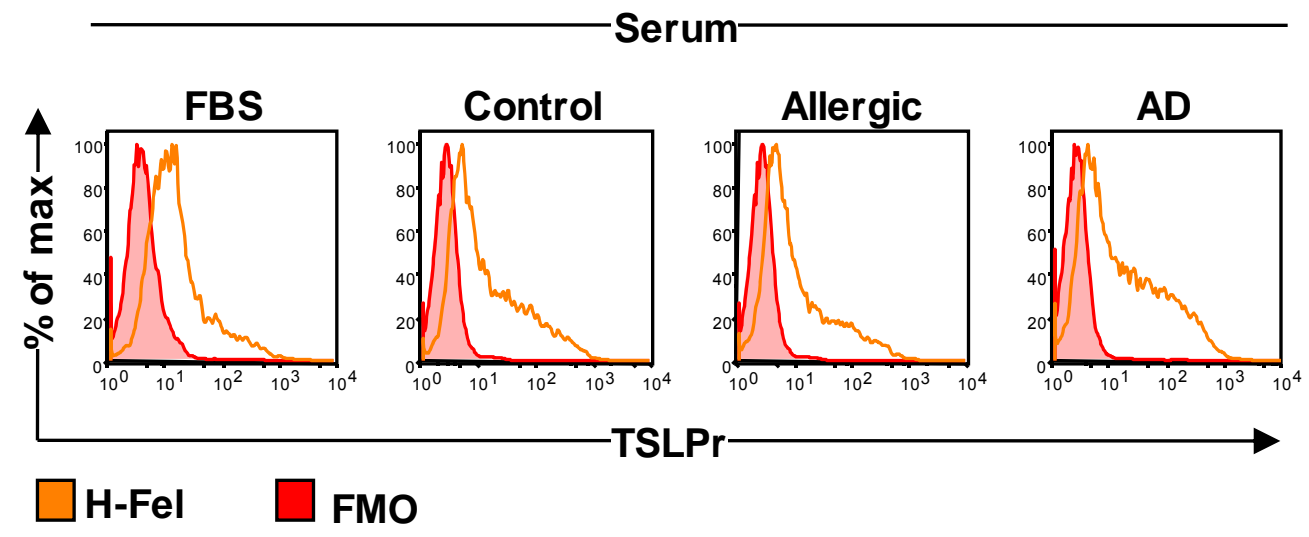


Next, we examined whether signaling was required for $\mathrm{H} 22-\mathrm{Fel} \mathrm{d} 1$ induced TSLPr upregulation. Pulsing atopic moDCs with $\mathrm{H} 22-\mathrm{Fel} \mathrm{d} 1$, but not non-receptor-targeted Fel d 1, led to the release of intracellular calcium supporting a role for signaling (Figure 3.13A). The tyrosine kinase, Syk and the src-related tyrosine kinases (SRTK) associate with the FcR $\gamma$ chain ITAM domain (159-161). These kinases can be inhibited by piceatannol and PP2 respectively. In the presence of these inhibitors there was a paradoxical increase in H22-Fel d 1-induced TSLPr expression in atopic moDCs and this was most evident when SRTKs were inhibited suggesting a negative regulatory role for these molecules (Figure 3.13B). To further investigate FcyRI signaling we tested the effects of inhibiting downstream components of FcyRI signaling. Inhibition of PI3K or PKC markedly reduced TSLPr expression in atopic moDCs in a dose-dependent manner (Figure 3.13B and C). In non-atopic moDCs, inhibition of SRTKs and PI3K had similar effects on H22-Fel d 1-induced TSLPr expression as in atopic cells. By contrast, inhibition of PKC markedly enhanced TSLPr expression. Moreover, inhibition of Syk had no effect or else reduced TSLPr expression (Figure 3.13C). Thus, PI3K positively regulates H22-Fel d 1-induced TSLPr upregulation in both atopic and non-atopic cells; however, there is a differential regulatory role for PKC and Syk kinase depending on atopic status.

TSLP Receptor is Weakly Induced by Low Dose LPS.

Several studies support the view that LPS can act as a Th2 adjuvant in allergic models (162-165). Thus, we examined whether LPS contributed to 
Figure 3.13 H22-Fel d 1 Induces TSLP Receptor in Atopic moDCs Through a Pathway Which is Regulated by PI3K and PKC.

A. Release of intracellular calcium was assessed by loading cells with Indo-1 and measuring the ratio of free to bound calcium after addition of antigen (indicated by vertical line). Data is representative of 4 atopic subjects. B. MoDCs were incubated with inhibitors for 1 hour before pulsing with H22-Fel d 1and analyzing by flow cytometry (24 hours). Inhibitors tested were piceatannol (Syk kinase inhibitor); PP2 (src-related tyrosine kinase inhibitor); Ly294002 (PI3K inhibitor); staurosporine (PKC inhibitor). Data is representative of 4 atopic subjects. C. Differential effects of kinase inhibitors on H22-Fel d 1-induced TSLPr expression in atopic and non-atopic moDCs. Data is representative of 4 subjects. 
A

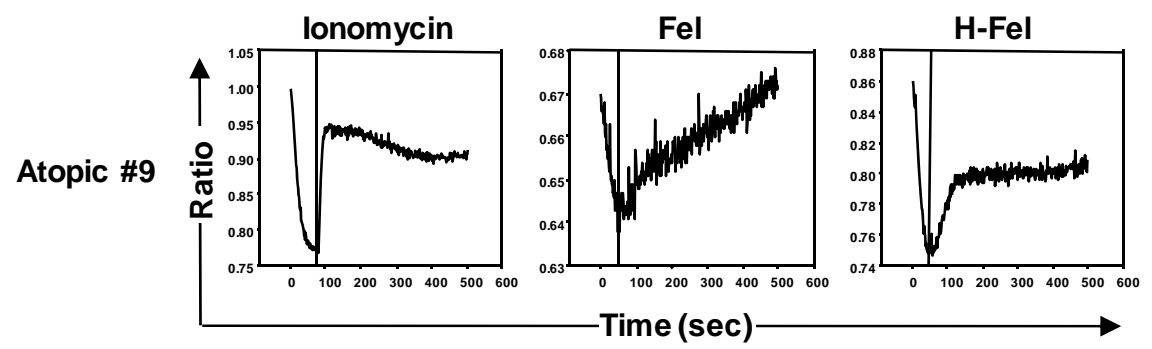

B Atopic \#1

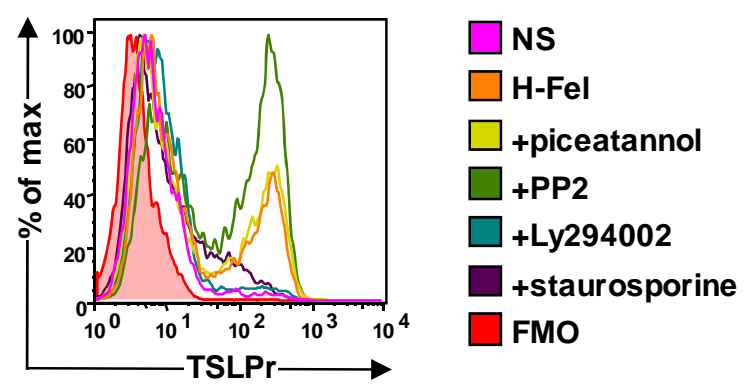

C

$+\mathrm{H}-\mathrm{Fel}$

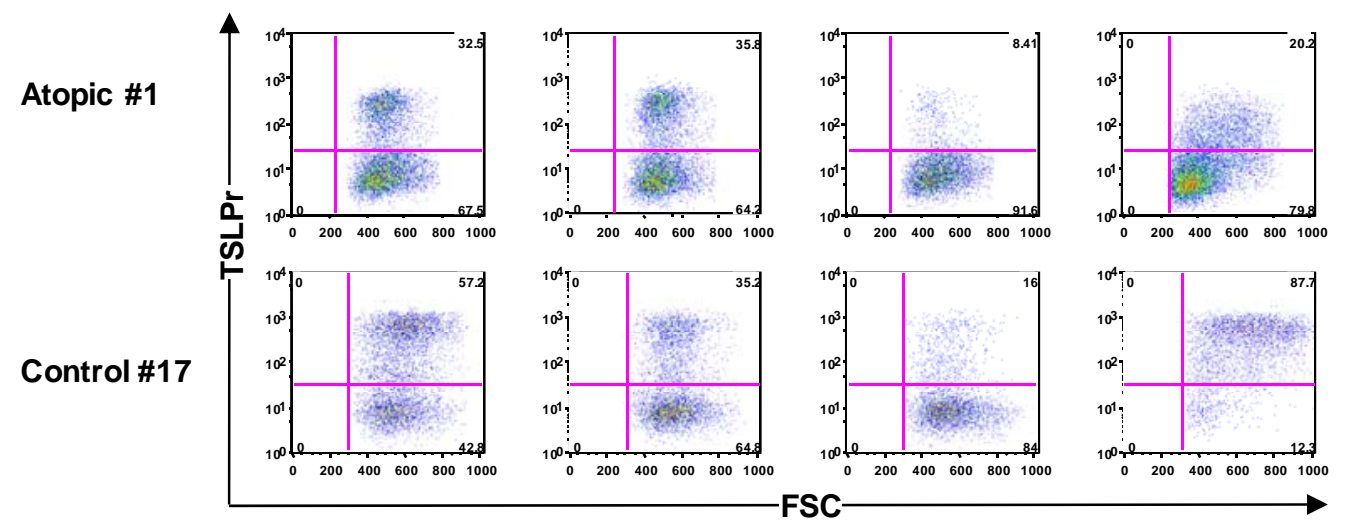


TSLPr upregulation mediated by $\mathrm{H} 22-\mathrm{Fel} \mathrm{d} 1$. The preparation of $\mathrm{H} 22-\mathrm{Fel} \mathrm{d} 1$ used contained low levels of endotoxin ( $4 \mathrm{EU} / \mathrm{ml}$ working concentration). Equivalent levels of LPS (5 EU/ml) weakly induced TSLPr expression as compared with H22-Fel d 1 in atopic moDCs (Figure 3.14A). Moreover, high dose LPS (10,000 EU/ml) failed to upregulate TSLPr to levels comparable with H22-Fel d 1 in atopic moDCs (Figure 3.14B). Since LPS binds to TLR4, we examined whether signaling through this pathway was involved in LPS-induced TSLPr upregulation in atopic moDCs. Inhibition of PI3K, a negative regulator of TLR-mediated responses, enhanced LPS-induced TSLPr expression in these cells (Figure 3.14C). Thus, while LPS has the capacity to induce TSLPr expression, it mediates its effects through a distinct pathway to $\mathrm{H} 22-\mathrm{Fel} \mathrm{d} 1$. Inhibition of PI3K and PKC in Atopic MoDCs Abolishes the Th2-Promoting Effect of TSLP.

Since inhibition of PI3K and PKC reduced TSLPr expression in atopic moDCs, we postulated that inhibiting these molecules in atopic moDCs would block the Th2-promoting effect of TSLP in cultures stimulated with H22-Fel d 1. Inhibition of PI3K or PKC selectively abolished IL-4+ T cells in cultures stimulated with atopic moDCs which had been primed with H22-Fel d 1 in the presence of TSLP. This included those IL-4+ T cells which co-expressed IFN- $\gamma$ (Figure 3.15). Inhibition of PI3K or PKC also markedly reduced IL-4-expressing cells induced by H22-Fel d 1 alone but had no effect on IL-4-expressing cells induced by TSLP 
Figure 3.14 TSLP Receptor is Weakly Induced by Low Dose LPS.

A. Effect of low dose LPS and H22-Fel d 1 on TSLP receptor expression in moDCs. Cells were pulsed with H22-Fel d 1 or LPS (5 EU/ml) and TSLP receptor was analyzed at 24 hours by flow cytometry. Data is representative of 2 experiments. B. Comparison of TSLP receptor expression induced by high dose LPS $(10,000 \mathrm{EU} / \mathrm{ml})$ and H22-Fel d 1 on atopic moDCs. Cells were prepared and analyzed as for $A$. Data is representative of $4 A D$ subjects. C. Effect of inhibiting PI3K on TSLP receptor expression induced by high dose LPS. Cells were incubated with inhibitor for 1 hour prior to pulsing with LPS and analyzing as for A. Data is representative of 2 experiments. 
A $\quad$ Atopic \#1

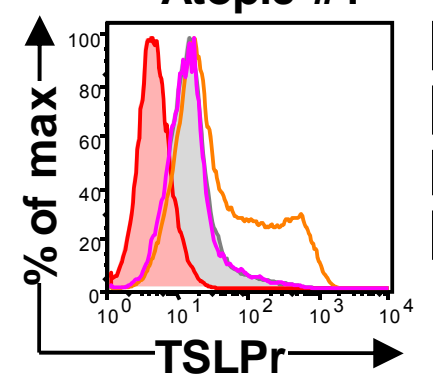

NS

$\square \mathrm{H}-\mathrm{Fel}(4 \mathrm{EU} / \mathrm{ml})$

$\square$ LPS (5 EU/mI)

$\square$ FMO

B

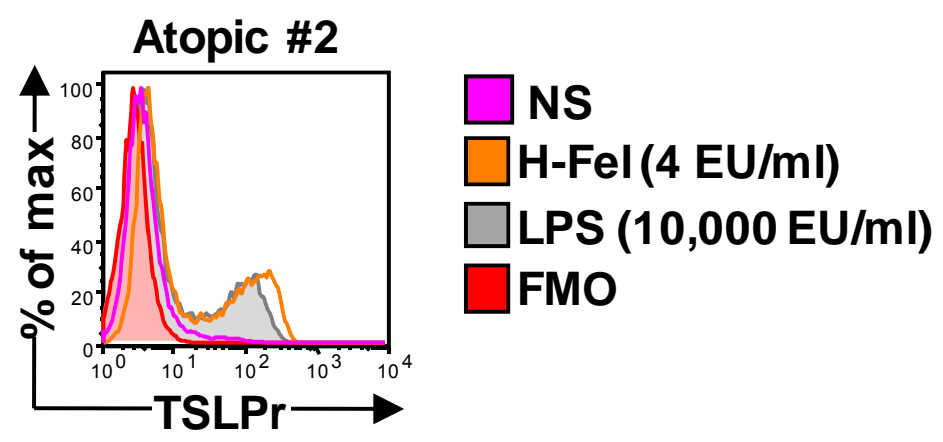

C

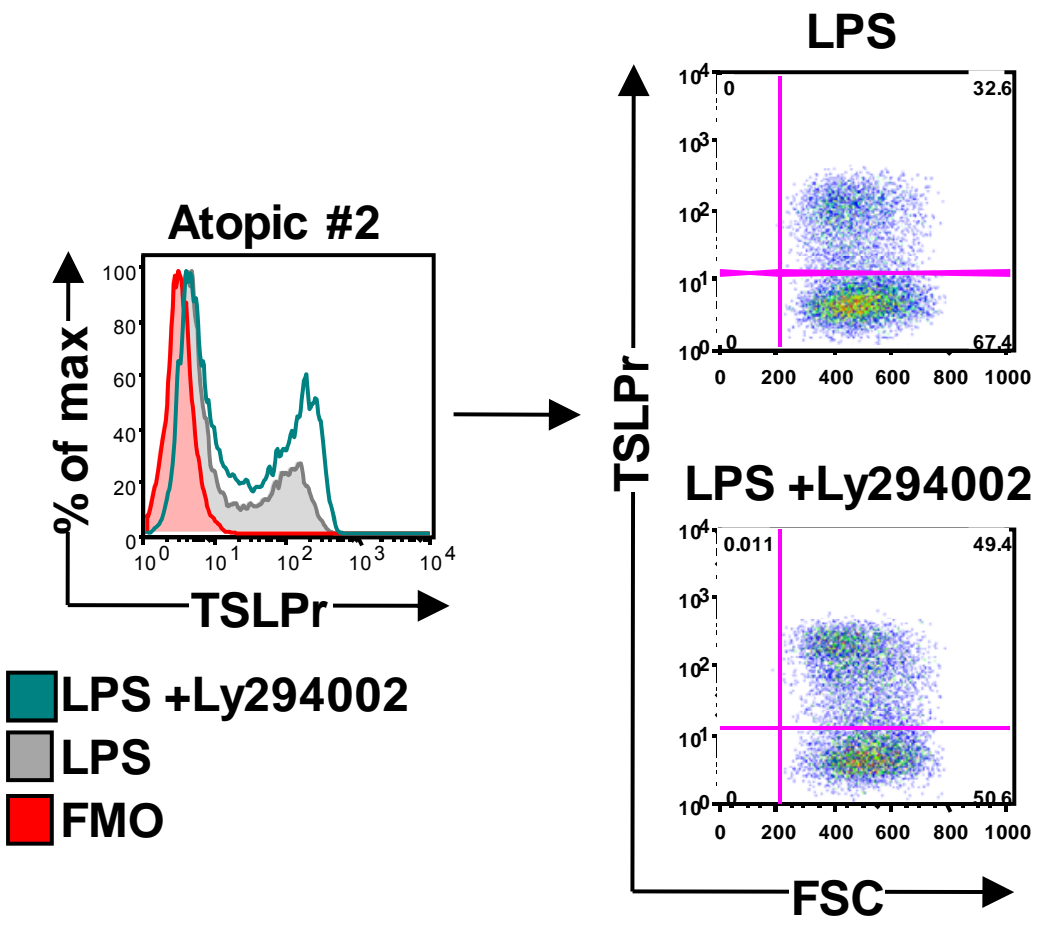


(Figure 3.15). These findings underscore the pivotal role of PI3K and PKC in mediating the Th2-promoting effects of TSLP triggered by H22-Fel d 1.

\section{Discussion}

We have identified a novel immune pathway which amplifies Th2 responses in humans. Specifically, we have demonstrated that targeting cat allergen to FcyRI upregulates expression of TSLPr in atopic moDCs. Upregulation of TSLPr was most pronounced in moDCs from atopic patients and Th2 responses triggered by $\mathrm{H} 22-\mathrm{Fel} \mathrm{d} 1$ were markedly amplified in the presence of TSLP in these individuals. These findings indicated that receptor-targeted allergen potentiates the Th2-promoting effect of TSLP through TSLPr upregulation. In support of this theory, we further showed that TSLPr upregulation was positively regulated by PI3K and PKC in atopic moDCs and inhibition of these molecules abolished the Th2-promoting effect of TSLP elicited by $\mathrm{H} 22-\mathrm{Fel} \mathrm{d} 1$. Our study is significant for several reasons: First, we have established differential effects of TSLP depending on allergic status; Second, we have shown that TSLP can exert Th2-promoting properties in an unconventional OX40L-independent manner; Finally, we have provided the first evidence of a link between $\mathrm{F} c \gamma \mathrm{R}$-mediated events and regulation of cytokine receptor expression in dendritic cells. It is well established that a variety of cytokines can modulate FcyR expression, through cytokine-mediated signaling pathways (166168). Our findings now show that the pathway linking $F c \gamma$ receptors to cytokinemediated pathways is bi-directional. 
Figure 3.15 Inhibition of PI3K and PKC Abolishes the Th2-Promoting Effect of TSLP.

Atopic moDCs were incubated with Ly294002 or staurosporine prior to pulsing with different stimuli. Cells were then washed and cultured with $\mathrm{CD} 4^{+} \mathrm{T}$ cells for 7 days. Intracellular cytokine expression was analyzed by flow cytometry. Dot plots show the phenotype of gated $\mathrm{CD} 4^{+} \mathrm{T}$ cells. 


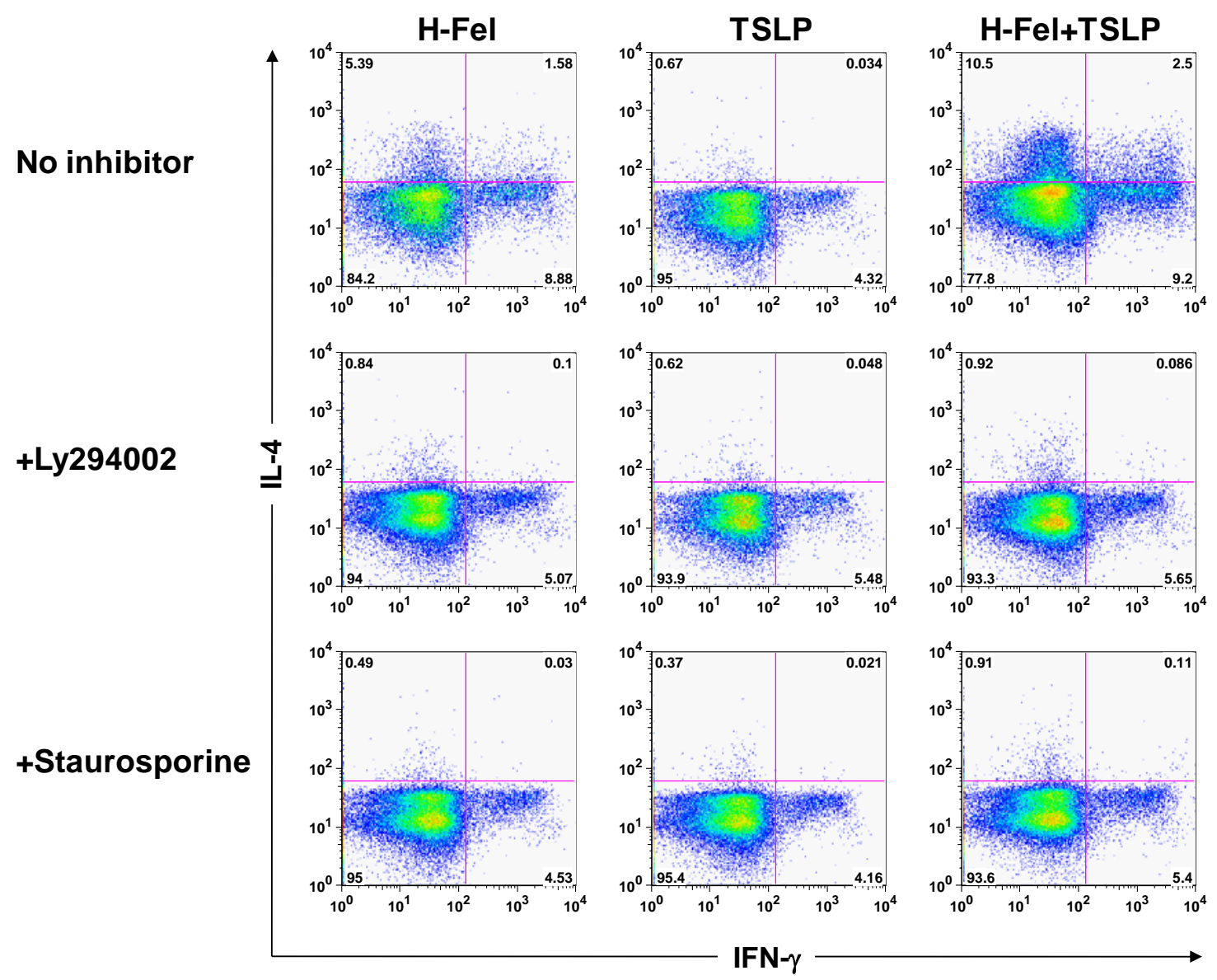


Atopic moDCs are equipped to rapidly upregulate TSLPr in response to $\mathrm{H} 22$ Fel $\mathrm{d} 1$ and this process is positively regulated by PI3K and PKC. PI3K has been shown to act downstream of Syk kinase in FcyRI signaling (169). Thus, we expected that inhibition of Syk kinase would reduce TSLPr expression in a similar manner to that observed when $\mathrm{PI} 3 \mathrm{~K}$ was inhibited. However, while inhibition of Syk resulted in a variable decrease in TSLPr expression in non-atopic moDCs, expression of TSLPr was actually enhanced in atopic cells. These findings suggest that PI3K regulates TSLPr expression independently of Syk in atopic moDCs. This could occur through perturbed Fc $\gamma \mathrm{RI}$ signaling or else through activation of an alternate FcyRI-mediated pathway. Consistent with the latter scenario, it was recently shown that Fcy receptor signaling can occur independently of Syk through residues outside the ITAM domain (169).

Similar to Syk kinase, PKC exerted a differential regulatory role on $\mathrm{H} 22-$ Fel d 1-induced TSLPr expression depending on atopic status. Specifically, PKC acted as a negative regulator in non-atopic moDCs, but as a positive regulator in atopic cells. A negative regulatory role for PKC in H22-Fel d 1-induced TSLPr expression is also observed in THP-1 monocytes (Hulse and Woodfolk, unpublished observations). While it is well established that PKC plays a key role in internalization of FcyRI in monocytes (170), little is known about how this may translate to dendritic cells. It is tempting to speculate that the differential regulatory role of PKC on TSLPr expression in atopic versus non-atopic cells relates to enhanced receptor internalization in atopic cells owing to increased surface density of Fc receptors. Alternatively, dysregulated signaling in atopic 
cells may subvert the negative regulatory role of PKC that operates in nonatopic cells.

LPS only weakly induced TSLPr in atopic moDCs; however, several lines of evidence indicate that our observations do not simply reflect the effects of endotoxin acting alone: (1) Non-receptor-targeted allergen which contains equivalent levels of endotoxin to $\mathrm{H} 22-\mathrm{Fel} \mathrm{d} 1$ is much less efficient at inducing TSLPr expression in atopic moDCs; (2) High dose LPS (10,000 EU/ml) fails to induce levels of TSLPr expression comparable to H22-Fel d 1 in atopic moDCs; (3) Blocking PI3K enhances TSLPr expression induced by LPS, but not by H22Fel d 1. Since PI3K is a negative regulator of TLR-mediated pathways, this latter point supports the view that LPS-, but not H22-Fel d 1-induced upregulation of TSLPr, acts through the TLR4 pathway. Thus, if LPS is acting as an adjuvant in our system, it acts in a different manner.

Collectively, our data show that negative regulatory mechanisms operate to control TSLPr expression mediated by both Fc $\gamma$ R (via PKC or SRTKs) and TLRs (via PI3K). From an evolutionary standpoint, both of these receptor types evolved to eliminate pathogens. Thus, negative regulation of TSLPr by these receptors fits with a model designed to suppress non-protective Th2 responses to microorganisms. It is well established that AD patients are susceptible to skin infections and this has been attributed to cutaneous deficiencies in specific components of innate immunity. However, we propose an alternate paradigm whereby pathogens could trigger inappropriate Th2 responses in these patients through ligation of innate signaling receptors on DCs. 
Interestingly, TSLPr expression can be upregulated by non-receptortargeted allergen; however, this process is highly variable, much less efficient than for receptor-targeted allergen, and restricted to cells from atopic subejcts. We postulate that this effect is mediated through ligation of FcRs expressed at high density on atopic moDCs by IgE-or IgG-bound allergen. It has been shown that moDCs from atopic patients express enhanced levels of Fc\&RI, and this is driven by enhanced expression of the $\gamma$ chains of the receptor (171). Notably, these $\gamma$ chains can be shared among Fc\&RI and FcyRI. Moreover, high expression of $\mathrm{Fc \varepsilon RI}$ on dendritic cells from atopic subjects is pivotal to allergen uptake and T cell activation (172). Thus, dendritic cells from atopic individuals may be better equipped to upregulate TSLPr upon allergen encounter and drive Th2 responses in the presence of TSLP. Collectively, our findings provide insight into the nature of $\mathrm{T}$ cell responses to allergens in vivo. Specifically, it is widely observed that purified allergens are relatively weak inducers of Th2 responses in human-based in vitro systems, despite potent Th2-inducing properties in vivo. By examining in vitro $\mathrm{T}$ cell responses to $\mathrm{H} 22-\mathrm{Fel} \mathrm{d} 1$ in the presence of TSLP, thereby mimicking the cytokine milieu present at sites of allergic inflammation, the potent Th2-inducing effects of allergen are revealed in atopic subjects.

Consistent with this, we have recently confirmed that moDCs from atopic dermatitis patients primed with non-receptor-targeted dust mite allergen (Der $p$ 1) in the presence of TSLP, prime a robust Th2 response (unpublished observations). Thus, allergen binding to FcRs on DCs may provide the essential trigger for TSLP to act as a "master switch" for Th2 responses in vivo. 
Interestingly, the TSLP-mediated amplification of Th2 responses

triggered through $\mathrm{Fc} \gamma \mathrm{RI}$ was distinct from that previously described $(118,119)$.

Notably, it did not involve the OX40L/OX40 pathway and was not associated with production of the Th2-attracting chemokine, TARC/CCL17. It is possible that these observations relate to the use of moDCs in our system, rather than CD11c ${ }^{+}$ dendritic cells which have been used in previous studies $(118,119)$. Arguing against this, we showed that H22-Fel d 1 also enhanced TSLPr expression in freshly isolated myeloid dendritic cells. Regardless, this thesis establishes that TSLP can induce Th2 responses through a novel OX40L-independent pathway. Little is known about TSLP-mediated signaling events in human dendritic cells. While it is known that TSLP activates Stat5 in myeloid cells, other signaling molecules in this pathway are unknown $(109,173)$. Moreover, it appears that signaling events downstream of the TSLPr are complex with distinct pathways governing proliferation and Stat5 phosphorylation (173). From a clinical standpoint, Stat5 deficiency in humans leads to a profound eczematous phenotype (174). This raises the intriguing possibility that the effects of TSLP in atopic dermatitis do not involve Stat5.

Our findings have several major clinical implications. First, this report questions whether dendritic cell-targeted allergens will be clinically efficacious in atopic individuals. The route of vaccine administration will no doubt be a key factor to consider if TSLP-mediated effects are to be avoided. Second, thinking beyond the allergy field, it will be important to examine whether non-allergens targeted to $\mathrm{Fc} \gamma$ receptors have the capacity to upregulate TSLPr and thus, 
provide a new approach to treating Th1-driven disorders. Third, the identification of a novel pathway linking Fc $\gamma \mathrm{RI}$ to an OX40L-independent Th2promoting pathway paves the way to identify new TSLP-activated molecular targets for treating allergic disease. Finally and most importantly, our results strongly support the view that targeting PI3K and PKC could provide a new strategy for treating allergic disease. 


\section{Chapter IV-Conclusions and Future Directions}

\section{Synopsis}

The work outlined in this thesis describes the immunomodulatory properties of a novel allergen variant, $\mathrm{H} 22-\mathrm{Fel} \mathrm{d} 1$, at the $\mathrm{T}$ cell and dendritic cell level. Initially, we examined the effects of H22-Fel d 1 on moDCs from catallergic and non-allergic subjects (Chapter II). Stimulation of moDCs with H22Fel $d 1$ induced a semi-mature phenotype characterized by no change in expression of co-stimulatory molecules, but enhanced production of Th1associated cytokines and chemokines with no change in Th2-promoting factors. Subsequently, we showed that H22-Fel d 1-primed moDCs induced an increased frequency of IL-5+ and IL-10+ CD4 ${ }^{+} \mathrm{T}$ cells, and these included subtypes characteristic of regulatory T cells. Interestingly, the effects of H22-Fel d 1 on $\mathrm{T}$ cell responses were restricted to cat-allergic subjects. The repertoire of $C D 4^{+} \mathrm{T}$ cells induced by H22-Fel d 1 was distinct from that induced by Fel $\mathrm{d} 1$ in modified Th2 responders and included more IL-10+ subtypes than that induced by the prototypic dust mite allergen, Der $p$ 1. Collectively, these data suggested that H22-Fel d 1 may provide a novel therapeutic molecule for treating cat-allergic subjects.

Next, we investigated whether $\mathrm{H} 22-\mathrm{Fel} \mathrm{d} 1$ had the potential to induce protective $T$ cell subsets in the presence of a cytokine milieu akin to that encountered in vivo in allergic subjects. To do this, we examined the effects of $\mathrm{H} 22-\mathrm{Fel} \mathrm{d} 1$ on the T cell repertoire induced in the presence of TSLP (chapter III). 
In contrast to previous reports, TSLP was a relatively weak inducer of IL-4+ T cells irrespective of allergic status, though there was a trend towards increased Th2 induction in highly atopic individuals. However, moDCs stimulated with both H22-Fel d 1 and TSLP induced a robust Th2 response, and this effect was most marked in subjects with atopic dermatitis. After verifying that TSLP was acting on moDCs in our system, we examined the mechanisms involved in driving Th2 amplification. Analysis of expression of surface co-stimulatory molecules and secreted cytokines by moDCs stimulated with $\mathrm{H} 22-\mathrm{Fel} \mathrm{d} 1$ either with or without TSLP failed to identify candidate molecules which may play a role in this process. Given the magnitude of the Th2 response generated, we postulated that H22-Fel d 1 promoted the effects of TSLP by acting on the TSLP pathway. It was previously shown that TSLP induced Th2 responses in an OX40L-dependent manner (119). However, there was no effect of TSLP on the levels of expression of OX40L induced by H22-Fel d 1, and Th2 responses were not inhibited by OX40L blockade. Interestingly, atopic moDCs constitutively expressed TSLPr and H22-Fel d 1 enhanced expression of this receptor, thereby providing a mechanism for amplification of the TSLP pathway. Extending this observation, we incorporated selective kinase inhibitors to investigate whether signaling through Fc $\gamma \mathrm{RI}$ was involved in TSLPr upregulation, and whether this played a role in the augmentation of Th2 responses. The bulk of previous work investigating signaling events triggered by $\mathrm{Fc} \gamma \mathrm{RI}$ was performed in cells other than dendritic cells; these include primary human monocytes or macrophages, and an array of human and murine cell lines. Because of this, existing 
knowledge regarding mechanisms involved in Fc $\gamma \mathrm{RI}$ signaling in dendritic cells is scant. Nevertheless, we demonstrated that upregulation of TSLPr induced by $\mathrm{H} 22-\mathrm{Fel} \mathrm{d} 1$ on moDCs from atopic subjects was positively regulated by both PI3K and PKC. By contrast, PKC negatively regulated TSLPr expression in moDCs from non-atopic subjects. Extending these results to the T cell level, inhibition of PI3K or PKC abolished IL-4+ T cells induced by H22-Fel d 1 in the presence of TSLP. Collectively, these results strongly support the view that H22Fel d 1 potentiates the Th2-promoting effects of TSLP through FcyRI signaling.

Our findings suggest that TSLPr upregulation involves components of different signaling pathways. Characterization of a novel Th2 regulatory pathway linking Fc $\gamma R$ I to TSLPr and consequent TSLP-driven events has important clinical implications for dendritic cell-based vaccines related to allergy. In addition, it identifies dendritic cell signaling components which could provide therapeutic targets for allergic disease.

Model

Based on our results, we propose a model whereby engagement of $\mathrm{H} 22-$ Fel $d 1$ triggers signaling through Fc $\gamma \mathrm{RI}$ on dendritic cells in atopic subjects. This leads to upregulation of TSLPr surface expression that primes these cells to respond to TSLP (Figure 4.1). Signaling pathways mediated by $F c \gamma R I$ in dendritic cells include PI3K and PKC. These pathways may play a role in the uptake and processing of $\mathrm{H} 22-\mathrm{Fel} \mathrm{d} \mathrm{1,} \mathrm{but} \mathrm{also} \mathrm{directly} \mathrm{upregulate} \mathrm{TSLPr}$ expression. Consequently, TSLP-mediated signaling enhances the Th2-inducing 
Figure 4.1 Model of H22-Fel d 1-Induced TSLPr Expression and Th2 Augmentation in Atopic Subjects.

Engagement of $\mathrm{H} 22-\mathrm{Fel} \mathrm{d} 1$ triggers signaling through $\mathrm{F} \gamma \gamma \mathrm{RI}$ on dendritic cells in atopic subjects. This leads to upregulation of TSLPr surface expression which primes cells to respond to TSLP. Signaling pathways mediated by FcyRI in this system include PI3K and PKC, which could act through distinct pathways. These pathways may play a role in the uptake and processing of $\mathrm{H} 22-\mathrm{Fel} \mathrm{d} 1$, but also directly upregulate TSLPr expression. Consequently, TSLP-mediated signaling enhances the Th2-inducing properties of the antigen presenting cell in an OX40L-independent manner, which results in increased T cell expression of IL-4 and amplification of Th2 responses. 


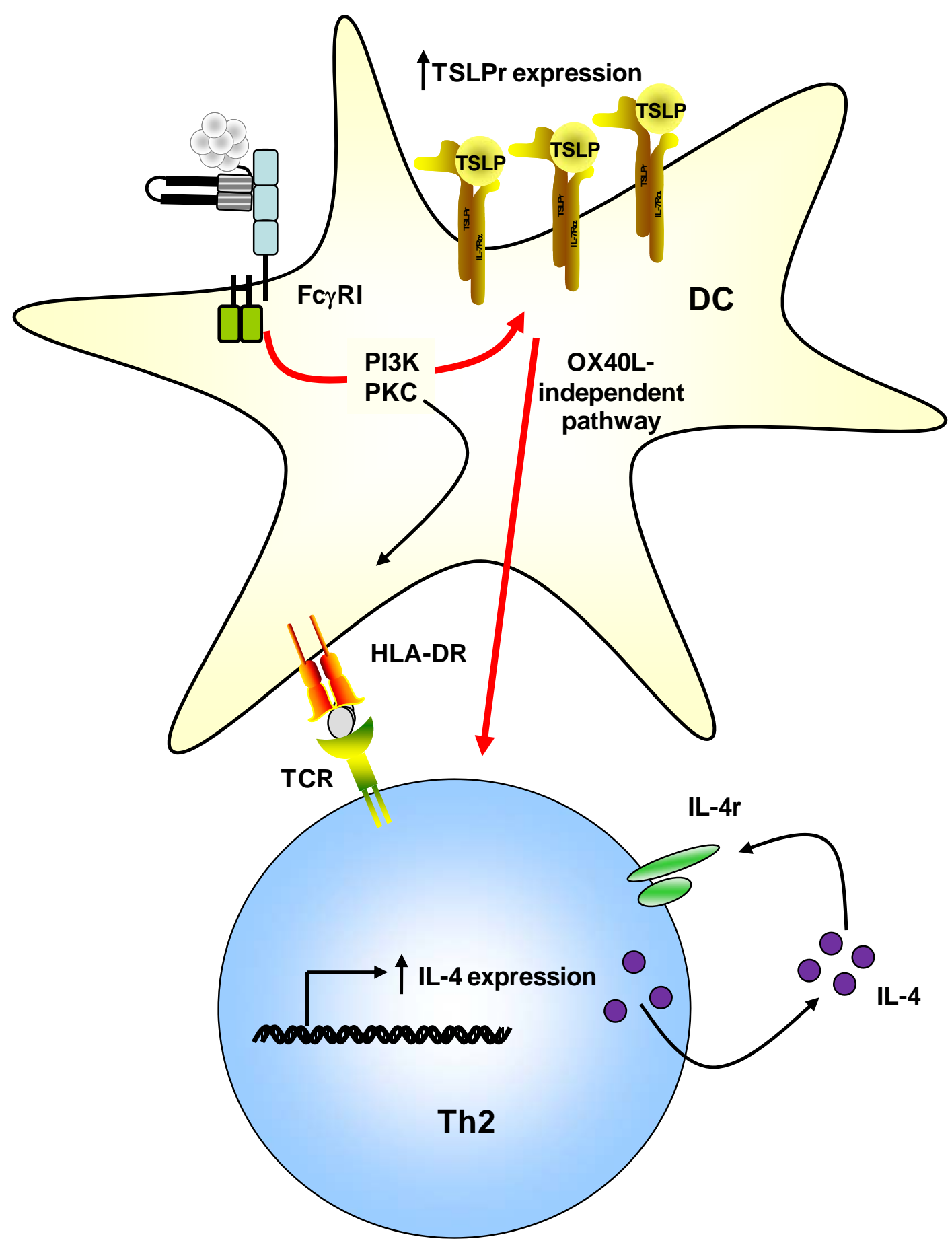


properties of the antigen presenting cell. In contrast to this, $\mathrm{H} 22-\mathrm{Fel} \mathrm{d} 1$ induced signaling in moDCs from non-atopic subjects leads to a more modest upregulation of TSLPr, and is negatively regulated by PKC. This suggests that negative regulatory mechanisms operate in non-atopic moDCs to inhibit TSLPr expression in response to FcyRl-mediated signals (Figure 4.2). Future investigations should focus on elucidating epigenetic variations between moDCs from atopic and non-atopic subjects that could account for the observed differences in their ability to upregulate TSLPr expression and selectively amplify Th2 responses. These are discussed in detail below.

\section{Future Directions}

Examine accessibility of the ts/pr gene locus in moDCs from atopic and nonatopic donors.

Work performed in this thesis identified positive and negative regulatory elements with evidence of differential roles for specific signaling components. Yet, the mechanisms that underly the ability for PKC to positively or negatively regulate expression of TSLPr in atopic and non-atopic cells are unclear. We expect that these singaling molecules activate transcription factors involved in TSLPr expression. However, the key difference between atopic and non-atopic moDCs may lie in the ability of transcription factors to bind to and activate the ts/pr locus. Chromatin accessibility is a key factor in dictating whether a particular gene will be expressed and is determined by chemical modifications of 
the histone proteins that are required for proper chromatin structure. These epigenetic changes involve methylation, acetylation, ubiquitination and

Figure 4.2 Differential Effects of Selective Kinase Inhibitors on H22-Fel d 1Induced TSLPr Expression in Atopic and Non-Atopic moDCs.

SRTKs have a negative regulatory effect and PI3K has a positive regulatory effect on H22-Fel d 1-induced TSLPr expression, regardless of atopic status. However, in moDCs from non-atopic donors, Syk has a positive regulatory effect and PKC has a negative regulatory effect. The effects of Syk and PKC are reversed in moDCs from atopic subjects suggesting dysregulated control of $\mathrm{H} 22-$ Fel d 1-TSLPr in atopic subjects. 
Atopic moDC

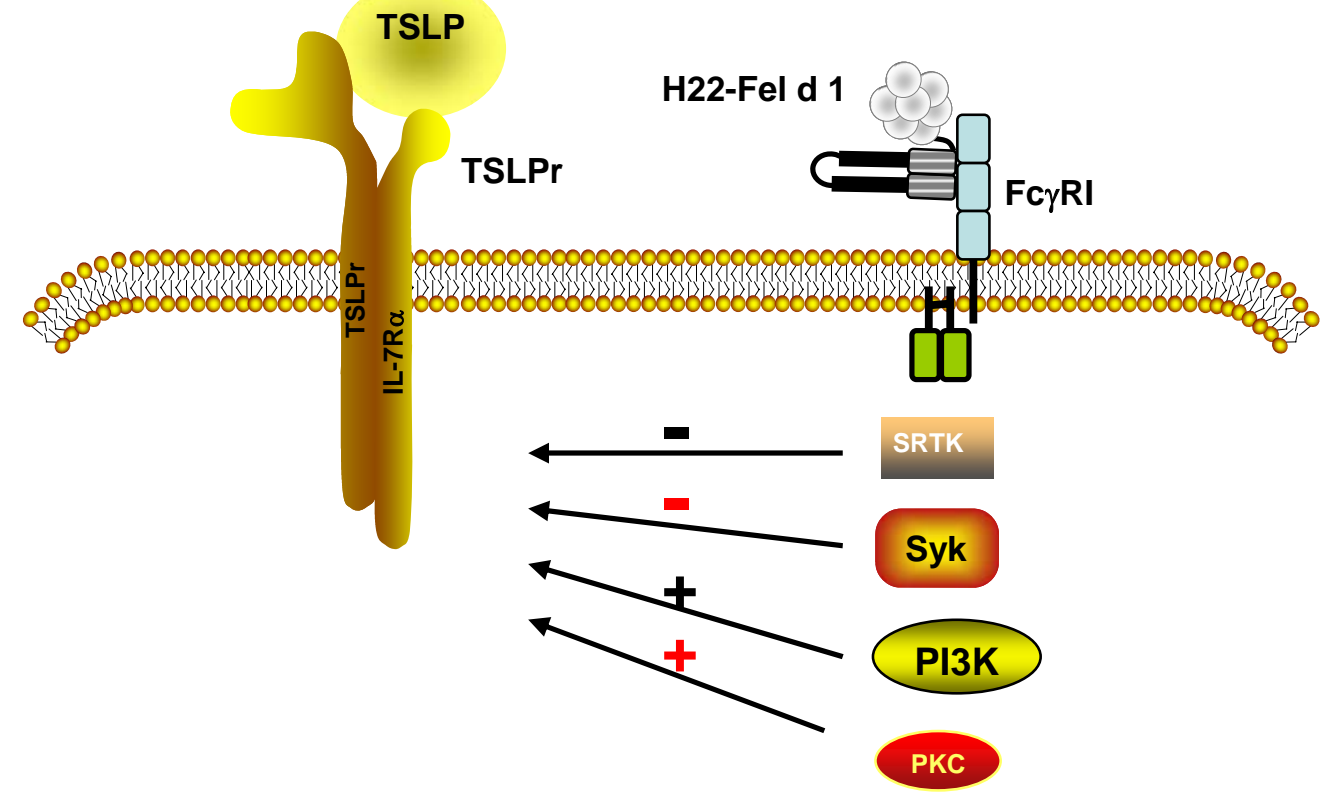

Non-atopic

moDC

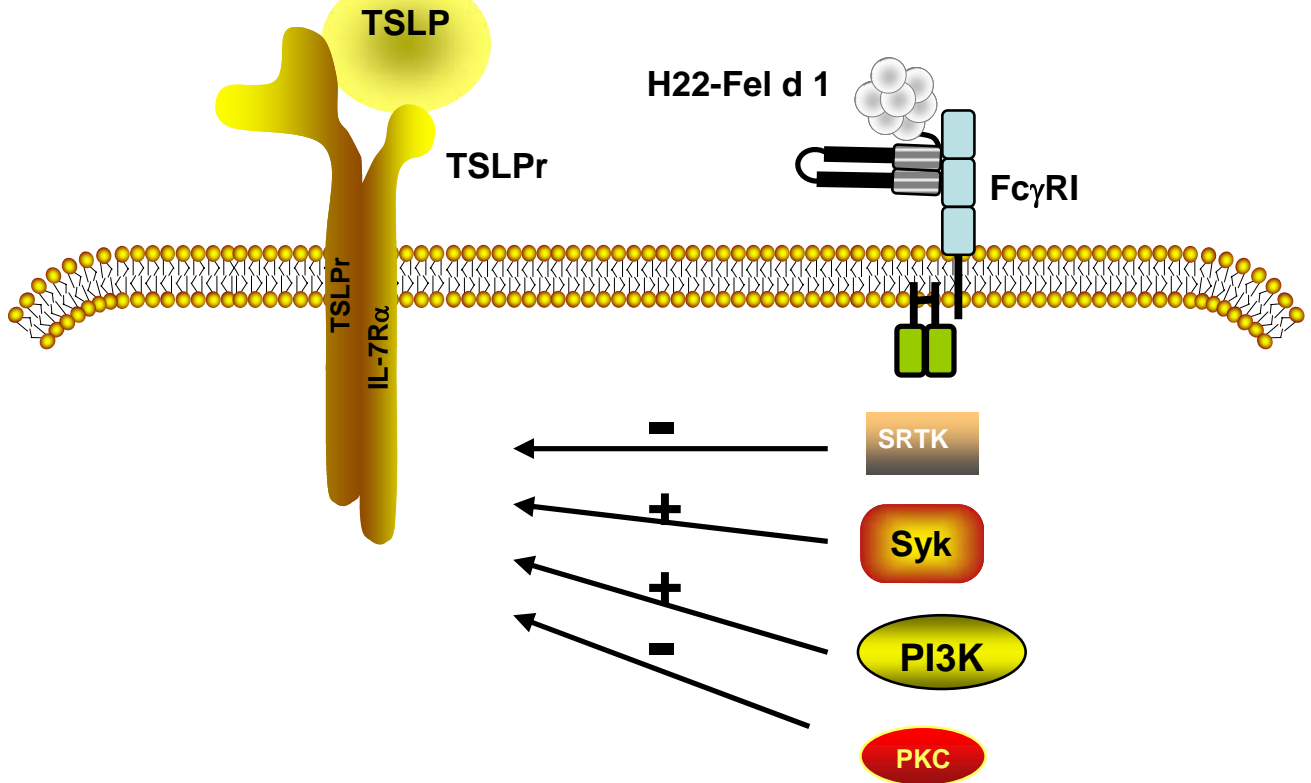


phosphorylation of amino acids within the histone tails or amino-terminal ends of histone proteins. For example, acetylation of histones $\mathrm{H} 3$ and $\mathrm{H} 4$ is associated with uncoupling of the DNA-histone interaction, which results in accessibility of that region. Similarly, methylation of lysine 4 of histone H3 is associated with active gene expression; however, methylation of lysine 9 of histone $\mathrm{H} 3$ is linked to gene silencing.

We hypothesize that the tslpr locus is more accessible in cells from atopic subjects compared with non-atopics. The relative availability of the ts/pr locus in cells from atopic and non-atopic subjects can be assessed using chromatin immunoprecipitation (ChIP) and quantitative RT-PCR techniques. Our data suggests that TSLPr is constitutively expressed on moDCs from atopic but not non-atopic subjects. Initial experiments could examine the baseline accessibility of the ts/pr locus by using unstimulated cells. Quantitation of the ts/pr gene by RT-PCR after pull-down with anti-acetylated histone H3 Abs will allow comparison of the baseline methylation state of that locus in atopic and nonatopic cells. These results could then be extended by priming atopic and nonatopic moDCs with $\mathrm{H} 22-\mathrm{Fel} \mathrm{d} 1$ and reassessing the relative levels of $t s / p r$ associated with acetylated histones after stimulation.

Based on our results we postulate that at baseline, the ts/pr locus is more accessible in cells from atopic donors, and that this availability is enhanced in the presence of H22-Fel d 1 compared with cells from non-atopics. However, it is possible that even if the baseline accessibility is higher in cells from atopic subjects, stimulation with $\mathrm{H} 22-\mathrm{Fel} \mathrm{d} 1$ has no additional epigenetic effect, but 
instead enhances activation of a transcription factor required for TSLPr expression in a PI3K- or PKC-dependent manner. If this is the case, we would expect to see no change in the accessibility of the ts/pr locus in response to $\mathrm{H} 22$ Fel d 1. ChIP analysis which incorporates Abs for methylated histones could also be performed to confirm results.

Investigation of the transcription factors involved in tslpr gene expression.

Epigenetic modifications alter the accessibility of genes to transcription factors that are required for the expression of the gene. Once the role of epigenetic modifications of the ts/pr locus in regulating ts/pr expression has been determined, it will be important to characterize which transcription factors bind to this promoter region and induce expression. Promoter regions of genes contain many regulatory elements that are critical for gene expression. Investigation of the promoter region of the tslpr gene could yield valuable information regarding putative binding sites for known transcription factors. Since, differences in TSLPr expression between atopic and non-atopic cells may be due to epigenetics, we would not expect to find a difference in the requirement for specific transcription factors in cells based on their atopic status. However, elucidation of which transcription factors are required for tslpr expression will allow further understanding of the regulation of TSLPr expression in general.

To date, the promoter region of the human tslpr gene has not been characterized. However, as mentioned in chapter I, the human TSLPr is $24 \%$ homologous to the IL-2 receptor common $\gamma$ chain $\left(\gamma_{c}\right)$, and this could provide 
insight into putative transcription factors that may also play a role in ts/pr gene expression (109). It has been reported that the 5 ' promoter region of the $\gamma_{c}$ contains consensus Ets binding sites (EBS)(CCCCTTCG) that associate with the transcription factors GABP and Elf-1 $(175,176)$. We postulate that the promoter of the ts/pr gene also contains EBS that could activate transcription. Computer prediction algorithms could be used to scan the promoter region of the ts/pr locus in order to identify putative EBS motifs. If any EBS are identified, electrophoretic mobility shift assay (EMSA) could then be performed to confirm binding of known EBS transcription factors, such as GABP or Elf-1. Finally, the transcriptional activity of these proteins could be confirmed by luciferase assay in THP-1 cells (177).

Due to the sequence homology between the $\gamma_{c}$ and ts/pr genes, we would predict that these genes are regulated by similar mechanisms, which overate through EBS domains. However, GABP and Elf-1 are only two examples of many possible transcription factors which are known to bind to these sites. It may be necessary to investigate alternate EBS binding factors in order to identify a specific binding partner for the ts/pr promoter region. Moreover, it is also reasonable to assume that the reguation of the ts/pr gene is distinct from, or involves more regulatory sites than the $\gamma_{c}$. If no putative EBS regions are identified, or regions identified do not account for the transcriptional activity of the locus, further characterization of the tslpr promoter in order to identify other putative transcription factor binding sites will be necessary. 
Investigate the role of PI3K and PKC in activation of transcription factors required for ts/pr expression.

Our data suggest that both $\mathrm{PI} 3 \mathrm{~K}$ and $\mathrm{PKC}$ play a role in the regulation of TSLPr expression, and we hypothesize that they are responsible for the activation of a critical transcription factor. Once we have identified a transcription factor required for TSLPr expression, we would next investigate whether its activation is controlled by PI3K or PKC. We would not expect to find a differential role for PKC in transcription factor activation between atopic and non-atopic cells, since this may be controlled at the epigenetic level.

Activation of the EBS related transcription factors GABP and Elf-1 occurs via phosphorylation $(178,179)$. Initially, we would investigate the basal phosphorylation state of these proteins in unstimulated moDCs by immunoprecipitation and western blotting. These results could then be extended by incorporating Ly294002 or staurosporine to inhibit PI3K or PKC activity respectively. We would predict that inhibition of PI3K or PKC would decrease basal activation of GABP and Elf-1. Because H22-Fel d 1-primed moDCs upregulated expression of TSLPr, we could also investigate the effects of H22Fel $d 1$ on the phosphorylation state of GABP and Elf-1 in the presence or absence of Ly294002 or staurosporine. We would expect H22-Fel d 1 stimulation to enhance the activation of transcription factors required for ts/pr expression, and this would be decreased in the presence of Ly294002 or staurosporine. Alternatively, it is reasonable to assume that expression of the ts/pr gene is controlled by both positive and negative regulators of transcription. 
As such, we could also explore the complement of activating and repressing factors present, and how they may work together to ultimately determine gene transcription.

In summary, identification of the epigenetic and transcriptional machinery required for expression of the ts/pr gene could provide novel insights into the key differences between moDCs from atopic and non-atopic subject that ultimately result in amplification of Th2 responses. Furthermore, elucidation of these pathways will provide new strategies for treating allergic disease.

\section{Final Conclusions and Clinical Implications}

We have demonstrated that targeting the major cat allergen to $\mathrm{Fc} \gamma \mathrm{RI}$ using the novel allergen variant, $\mathrm{H} 22-\mathrm{Fel} \mathrm{d} 1$, induces a $\mathrm{T}$ cell cytokine repertoire in catallergic subjects that incorporates protective elements. However, the magnitude and quality of the $\mathrm{T}$ cell response triggered by $\mathrm{H} 22-\mathrm{Fel} \mathrm{d} 1$ are altered in the presence of the "pro-allergic" cytokine, TSLP. Specifically, Th2 responses are selectively increased through FcyRI-mediated events which enhance the TSLP pathway through upregulation of TSLP receptor. A key issue which remains is whether targeting allergens to $\mathrm{Fc} \gamma \mathrm{RI}$ is an appropriate strategy for treating allergic disease. This will depend on several factors including the dosing regimen and the capacity for TSLP and receptor-targeted allergen to exert a coordinated effect in vivo. In relation to the first point, numerous studies have established the clinical efficacy of conventional immunotherapy which involves injection of allergen extract in an updosing regimen $(62,64,79,86)$. Moving 
forward, it will be important to determine how the dosing regimen in vivo influences the quality of the $\mathrm{T}$ cell response induced by H22-Fel d 1 . In relation to the second point, TSLP is expressed in the skin lesions of patients with atopic dermatitis, and it has been reported to be constitutively expressed by skin keratinocytes (118). Thus, receptor-targeted allergen injected into the skin of patients with atopic dermatitis could, in theory, encounter dendritic cells which are actively responding to TSLP. Migration of these cells from the skin to regional lymph nodes could generate a robust Th2 response and homing of effector T cells back to the skin could then exacerbate inflammation. This process would obviously depend on the ability for dendritic cells to rapidly upregulate TSLPr in the skin in response to H22-Fel d 1 where TSLP is expressed. However, it is also possible that injection of receptor-targeted allergen into healthy skin where levels of TSLP are low would preclude these effects.

It should be pointed out that while moDCs stimulated with both $\mathrm{H} 22-\mathrm{Fel} \mathrm{d}$ 1 and TSLP induced a robust Th2 response, the Th1 cytokine IFN- $\gamma$, was also a feature of the T cell response generated. Specifically, a proportion of IL- $4^{+} \mathrm{T}$ cells (roughly a third) co-expressed IFN- $\gamma$, and the total frequency of IFN- $\gamma^{+} \mathrm{T}$ cells was increased compared with either stimulus alone, though this did not reach significance. Since Th1 skewing has been associated with a favorable treatment outcome in clinical trials of immunotherapy, it is difficult to predict whether the quality of the Th2 response induced by co-stimulated moDCs would translate to a protective or non-protective response in vivo. Unfortunately, small 
animal models will likely not be useful for addressing this question in light of the disappointing discrepancies between mice and men which have now been reported for a variety of potential therapeutics for allergic disease. Thus, it is likely that definitive answers may only be attained in large animal models or else in clinical trials.

We have characterized a novel pathway linking $F c \gamma R I$ signaling to upregulation of TSLPr expression and consequent TSLP-driven events. Inhibition of signaling components required for TSLPr upregulation essentially abolished Th2 responses. This finding is of major importance since it suggests that targeting FcR signaling components on dendritic cells may be a rational objective for treating Th2-driven inflammatory disorders. Conversely, targeting antigens to FcRs which modulate TSLPr expression could also have important implications for Th1-mediated diseases where enhanced Th2 responses would be favorable. Indeed, it has recently been shown in a mouse model of autoimmune diabetes that injection of TSLP-primed dendritic cells into prediabetic NOD mice was able to prevent induction of diabetes (180). Finally, elucidation of the mechanisms responsible for the differential upregulation of TSLPr expression in atopic cells could provide new molecular targets for the treatment of allergic diseases.

In conclusion, studies on $\mathrm{H} 22-\mathrm{Fel} \mathrm{d} 1$ described in this thesis reveal a novel pathway which regulates Th2 responses in atopic individuals. This work provides a springboard for studying new immune pathways which may be exploited in the treatment of allergic disease. 


\section{References}

1. Spergel, J.M., and A.S. Paller. 2003. Atopic dermatitis and the atopic march. J Allergy Clin Immunol 112:S118-127.

2. Rhodes, H.L., R. Sporik, P. Thomas, S.T. Holgate, and J.J. Cogswell. 2001. Early life risk factors for adult asthma: a birth cohort study of subjects at risk. $J$ Allergy Clin Immunol 108:720-725.

3. Rhodes, H.L., P. Thomas, R. Sporik, S.T. Holgate, and J.J. Cogswell. 2002. A birth cohort study of subjects at risk of atopy: twenty-two-year follow-up of wheeze and atopic status. Am J Respir Crit Care Med 165:176-180.

4. Gustafsson, D., O. Sjoberg, and T. Foucard. 2000. Development of allergies and asthma in infants and young children with atopic dermatitis--a prospective followup to 7 years of age. Allergy 55:240-245.

5. Kulig, M., R. Bergmann, U. Klettke, V. Wahn, U. Tacke, and U. Wahn. 1999. Natural course of sensitization to food and inhalant allergens during the first 6 years of life. J Allergy Clin Immunol 103:1173-1179.

6. Lau, S., R. Nickel, B. Niggemann, C. Gruber, C. Sommerfeld, S. Illi, M. Kulig, J. Forster, U. Wahn, M. Groeger, F. Zepp, W. Kamin, I. Bieber, U. Tacke, V. Wahn, C.P. Bauer, R. Bergmann, and E. von Mutius. 2002. The development of childhood asthma: lessons from the German Multicentre Allergy Study (MAS). Paediatr Respir Rev 3:265-272.

7. Ohshima, Y., A. Yamada, M. Hiraoka, K. Katamura, S. Ito, T. Hirao, H. Akutagawa, N. Kondo, A. Morikawa, and M. Mayumi. 2002. Early sensitization to house dust mite is a major risk factor for subsequent development of bronchial 
asthma in Japanese infants with atopic dermatitis: results of a 4-year followup study. Ann Allergy Asthma Immunol 89:265-270.

8. 1993. Severity scoring of atopic dermatitis: the SCORAD index. Consensus Report of the European Task Force on Atopic Dermatitis. Dermatology 186:2331.

9. Leung, D.Y., and T. Bieber. 2003. Atopic dermatitis. Lancet 361:151-160.

10. Carneiro, R., A. Reefer, B. Wilson, J. Hammer, T. Platts-Mills, N. Custis, and J. Woodfolk. 2004. T cell epitope-specific defects in the immune response to cat allergen in patients with atopic dermatitis. J Invest Dermatol 122:927-936.

11. Leung, D.Y., M. Boguniewicz, M.D. Howell, I. Nomura, and Q.A. Hamid. 2004. New insights into atopic dermatitis. J Clin Invest 113:651-657.

12. Reefer, A.J., S.M. Satinover, B.B. Wilson, and J.A. Woodfolk. 2007. The relevance of microbial allergens to the IgE antibody repertoire in atopic and nonatopic eczema. J Allergy Clin Immunol 120:156-163.

13. Bieber, T., H. de la Salle, A. Wollenberg, J. Hakimi, R. Chizzonite, J. Ring, D. Hanau, and C. de la Salle. 1992. Human epidermal Langerhans cells express the high affinity receptor for immunoglobulin E (Fc epsilon RI). J Exp Med 175:12851290.

14. von Bubnoff, D., N. Novak, S. Kraft, and T. Bieber. 2003. The central role of FcepsilonRI in allergy. Clin Exp Dermatol 28:184-187.

15. Ong, P.Y., and D.Y. Leung. 2006. Immune dysregulation in atopic dermatitis. Curr Allergy Asthma Rep 6:384-389.

16. Kay, A.B. 2005. The role of eosinophils in the pathogenesis of asthma. Trends Mol Med 11:148-152.

17. Holgate, S.T. 2008. Pathogenesis of asthma. Clin Exp Allergy 38:872-897. 
18. Kraft, M., R.J. Martin, S. Wilson, R. Djukanovic, and S.T. Holgate. 1999. Lymphocyte and eosinophil influx into alveolar tissue in nocturnal asthma. Am J Respir Crit Care Med 159:228-234.

19. Eder, W., M.J. Ege, and E. von Mutius. 2006. The asthma epidemic. N Engl J Med 355:2226-2235.

20. Bucchieri, F., S.M. Puddicombe, J.L. Lordan, A. Richter, D. Buchanan, S.J. Wilson, J. Ward, G. Zummo, P.H. Howarth, R. Djukanovic, S.T. Holgate, and D.E. Davies. 2002. Asthmatic bronchial epithelium is more susceptible to oxidantinduced apoptosis. Am J Respir Cell Mol Biol 27:179-185.

21. Kicic, A., E.N. Sutanto, P.T. Stevens, D.A. Knight, and S.M. Stick. 2006. Intrinsic biochemical and functional differences in bronchial epithelial cells of children with asthma. Am J Respir Crit Care Med 174:1110-1118.

22. Ibiapina Cda, C., E.S. Sarinho, P.A. Camargos, C.R. Andrade, and A.A. Cruz Filho. 2008. Allergic rhinitis: epidemiological aspects, diagnosis and treatment. J Bras Pneumol 34:230-240.

23. Settipane, R.J., G.W. Hagy, and G.A. Settipane. 1994. Long-term risk factors for developing asthma and allergic rhinitis: a 23-year follow-up study of college students. Allergy Proc 15:21-25.

24. Leynaert, B., C. Neukirch, S. Kony, A. Guenegou, J. Bousquet, M. Aubier, and F. Neukirch. 2004. Association between asthma and rhinitis according to atopic sensitization in a population-based study. J Allergy Clin Immunol 113:86-93.

25. Ciprandi, G., and G. Passalacqua. 2008. Allergy and the nose. Clin Exp Immunol 153 Suppl 1:22-26.

26. Braunstahl, G.J., and W. Fokkens. 2003. Nasal involvement in allergic asthma. Allergy 58:1235-1243. 
27. van Ree, R., W.A. van Leeuwen, I. Bulder, J. Bond, and R.C. Aalberse. 1999. Purified natural and recombinant Fel d 1 and cat albumin in in vitro diagnostics for cat allergy. J Allergy Clin Immunol 104:1223-1230.

28. Hilger, C., F. Grigioni, and F. Hentges. 1996. Sequence of the gene encoding cat (Felis domesticus) serum albumin. Gene 169:295-296.

29. Ichikawa, K., L.D. Vailes, A. Pomes, and M.D. Chapman. 2001. Molecular cloning, expression and modelling of cat allergen, cystatin (Fel d 3), a cysteine protease inhibitor. Clin Exp Allergy 31:1279-1286.

30. Smith, W., A.J. Butler, L.A. Hazell, M.D. Chapman, A. Pomes, D.G. Nickels, and W.R. Thomas. 2004. Fel d 4, a cat lipocalin allergen. Clin Exp Allergy 34:17321738.

31. Kaiser, L., T.C. Velickovic, D. Badia-Martinez, J. Adedoyin, S. Thunberg, D. Hallen, K. Berndt, H. Gronlund, G. Gafvelin, M. van Hage, and A. Achour. 2007. Structural characterization of the tetrameric form of the major cat allergen Fel $d$ 1. J Mol Biol 370:714-727.

32. Kaiser, L., H. Gronlund, T. Sandalova, H.G. Ljunggren, M. van Hage-Hamsten, A. Achour, and G. Schneider. 2003. The crystal structure of the major cat allergen Fel d 1, a member of the secretoglobin family. J Biol Chem 278:3773037735.

33. Kaiser, L., H. Gronlund, T. Sandalova, H.G. Ljunggren, G. Schneider, M. van Hage-Hamsten, and A. Achour. 2003. Production, crystallization and preliminary crystallographic study of the major cat allergen Fel d 1. Acta Crystallogr D Biol Crystallogr 59:1103-1105. 
34. Klug, J., H.M. Beier, A. Bernard, B.S. Chilton, T.P. Fleming, R.I. Lehrer, L. Miele, N. Pattabiraman, and G. Singh. 2000. Uteroglobin/Clara cell 10-kDa family of proteins: nomenclature committee report. Ann N Y Acad Sci 923:348-354.

35. Mukherjee, A.B., G.C. Kundu, G. Mantile-Selvaggi, C.J. Yuan, A.K. Mandal, S. Chattopadhyay, F. Zheng, N. Pattabiraman, and Z. Zhang. 1999. Uteroglobin: a novel cytokine? Cell Mol Life Sci 55:771-787.

36. Peri, A., E. Cordella-Miele, L. Miele, and A.B. Mukherjee. 1993. Tissue-specific expression of the gene coding for human Clara cell 10-kD protein, a phospholipase A2-inhibitory protein. J Clin Invest 92:2099-2109.

37. Pilon, A.L. 2000. Rationale for the development of recombinant human CC10 as a therapeutic for inflammatory and fibrotic disease. Ann N Y Acad Sci 923:280299.

38. Arbes, S.J., Jr., P.J. Gergen, L. Elliott, and D.C. Zeldin. 2005. Prevalences of positive skin test responses to 10 common allergens in the US population: results from the third National Health and Nutrition Examination Survey. J Allergy Clin Immunol 116:377-383.

39. Woodfolk, J.A. 2007. T-cell responses to allergens. J Allergy Clin Immunol 119:280-294; quiz 295-286.

40. Brusselle, G.G., J.C. Kips, J.H. Tavernier, J.G. van der Heyden, C.A. Cuvelier, R.A. Pauwels, and H. Bluethmann. 1994. Attenuation of allergic airway inflammation in IL-4 deficient mice. Clin Exp Allergy 24:73-80.

41. Foster, P.S., S.P. Hogan, A.J. Ramsay, K.I. Matthaei, and I.G. Young. 1996. Interleukin 5 deficiency abolishes eosinophilia, airways hyperreactivity, and lung damage in a mouse asthma model. J Exp Med 183:195-201. 
42. Li, X.M., B.H. Schofield, Q.F. Wang, K.H. Kim, and S.K. Huang. 1998. Induction of pulmonary allergic responses by antigen-specific Th2 cells. $J$ Immunol 160:1378-1384.

43. Lambert, A.L., D.W. Winsett, D.L. Costa, M.K. Selgrade, and M.I. Gilmour. 1998. Transfer of allergic airway responses with serum and lymphocytes from rats sensitized to dust mite. Am J Respir Crit Care Med 157:1991-1999.

44. Cohn, L., J.S. Tepper, and K. Bottomly. 1998. IL-4-independent induction of airway hyperresponsiveness by Th2, but not Th1, cells. J Immunol 161:38133816.

45. Kaminuma, O., A. Mori, K. Ogawa, A. Nakata, H. Kikkawa, K. Ikezawa, and H. Okudaira. 1999. Cloned Th cells confer eosinophilic inflammation and bronchial hyperresponsiveness. Int Arch Allergy Immunol 118:136-139.

46. Randolph, D.A., C.J. Carruthers, S.J. Szabo, K.M. Murphy, and D.D. Chaplin. 1999. Modulation of airway inflammation by passive transfer of allergen-specific Th1 and Th2 cells in a mouse model of asthma. J Immunol 162:2375-2383.

47. Prescott, S.L., C. Macaubas, B.J. Holt, T.B. Smallacombe, R. Loh, P.D. Sly, and P.G. Holt. 1998. Transplacental priming of the human immune system to environmental allergens: universal skewing of initial T cell responses toward the Th2 cytokine profile. J Immunol 160:4730-4737.

48. Prescott, S.L., C. Macaubas, T. Smallacombe, B.J. Holt, P.D. Sly, R. Loh, and P.G. Holt. 1998. Reciprocal age-related patterns of allergen-specific T-cell immunity in normal vs. atopic infants. Clin Exp Allergy 28 Suppl 5:39-44; discussion 50-31.

49. Wahn, U., S. Lau, R. Bergmann, M. Kulig, J. Forster, K. Bergmann, C.P. Bauer, and I. Guggenmoos-Holzmann. 1997. Indoor allergen exposure is a risk factor for 
sensitization during the first three years of life. J Allergy Clin Immunol 99:763769.

50. Sporik, R., S.P. Squillace, J.M. Ingram, G. Rakes, R.W. Honsinger, and T.A. Platts-Mills. 1999. Mite, cat, and cockroach exposure, allergen sensitisation, and asthma in children: a case-control study of three schools. Thorax 54:675-680.

51. Shevach, E.M. 2006. From vanilla to 28 flavors: multiple varieties of T regulatory cells. Immunity 25:195-201.

52. Seddiki, N., B. Santner-Nanan, J. Martinson, J. Zaunders, S. Sasson, A. Landay, M. Solomon, W. Selby, S.I. Alexander, R. Nanan, A. Kelleher, and B. Fazekas de St Groth. 2006. Expression of interleukin (IL)-2 and IL-7 receptors discriminates between human regulatory and activated T cells. J Exp Med 203:1693-1700.

53. Liu, W., A.L. Putnam, Z. Xu-Yu, G.L. Szot, M.R. Lee, S. Zhu, P.A. Gottlieb, P. Kapranov, T.R. Gingeras, B. Fazekas de St Groth, C. Clayberger, D.M. Soper, S.F. Ziegler, and J.A. Bluestone. 2006. CD127 expression inversely correlates with FoxP3 and suppressive function of human CD4+ T reg cells. J Exp Med 203:1701-1711.

54. Hartigan-O'Connor, D.J., C. Poon, E. Sinclair, and J.M. McCune. 2007. Human CD4+ regulatory T cells express lower levels of the IL-7 receptor alpha chain (CD127), allowing consistent identification and sorting of live cells. J Immunol Methods 319:41-52.

55. Platts-Mills, T., J. Vaughan, S. Squillace, J. Woodfolk, and R. Sporik. 2001. Sensitisation, asthma, and a modified Th2 response in children exposed to cat allergen: a population-based cross-sectional study. Lancet 357:752-756. 
56. Hesselmar, B., B. Aberg, B. Eriksson, B. Bjorksten, and N. Aberg. 2003. High-dose exposure to cat is associated with clinical tolerance--a modified Th2 immune response? Clin Exp Allergy 33:1681-1685.

57. Perzanowski, M.S., E. Ronmark, T.A. Platts-Mills, and B. Lundback. 2002. Effect of cat and dog ownership on sensitization and development of asthma among preteenage children. Am J Respir Crit Care Med 166:696-702.

58. Custovic, A., B.M. Simpson, A. Simpson, C.L. Hallam, H. Marolia, D. Walsh, J. Campbell, and A. Woodcock. 2003. Current mite, cat, and dog allergen exposure, pet ownership, and sensitization to inhalant allergens in adults. $J$ Allergy Clin Immunol 111:402-407.

59. Ownby, D.R., C.C. Johnson, and E.L. Peterson. 2002. Exposure to dogs and cats in the first year of life and risk of allergic sensitization at 6 to 7 years of age. Jama 288:963-972.

60. Reefer, A.J., R.M. Carneiro, N.J. Custis, T.A. Platts-Mills, S.S. Sung, J. Hammer, and J.A. Woodfolk. 2004. A role for IL-10-mediated HLA-DR7-restricted T celldependent events in development of the modified Th2 response to cat allergen. $\mathrm{J}$ Immunol 172:2763-2772.

61. Nelson, H.S. 2007. Allergen immunotherapy: where is it now? J Allergy Clin Immunol 119:769-779.

62. Larche, M., C.A. Akdis, and R. Valenta. 2006. Immunological mechanisms of allergen-specific immunotherapy. Nat Rev Immunol 6:761-771.

63. Nouri-Aria, K.T., P.A. Wachholz, J.N. Francis, M.R. Jacobson, S.M. Walker, L.K. Wilcock, S.Q. Staple, R.C. Aalberse, S.J. Till, and S.R. Durham. 2004. Grass pollen immunotherapy induces mucosal and peripheral IL-10 responses and blocking IgG activity. J Immunol 172:3252-3259. 
64. Jutel, M., M. Akdis, K. Blaser, and C.A. Akdis. 2006. Mechanisms of allergen specific immunotherapy--T-cell tolerance and more. Allergy 61:796-807.

65. Radulovic, S., M.R. Jacobson, S.R. Durham, and K.T. Nouri-Aria. 2008. Grass pollen immunotherapy induces Foxp3-expressing CD4+ CD25+ cells in the nasal mucosa. J Allergy Clin Immunol 121:1467-1472, 1472 e1461.

66. Wilson, D.R., A.M. Irani, S.M. Walker, M.R. Jacobson, I.S. Mackay, L.B. Schwartz, and S.R. Durham. 2001. Grass pollen immunotherapy inhibits seasonal increases in basophils and eosinophils in the nasal epithelium. Clin Exp Allergy 31:1705-1713.

67. Durham, S.R., V.A. Varney, M. Gaga, M.R. Jacobson, E.M. Varga, A.J. Frew, and A.B. Kay. 1999. Grass pollen immunotherapy decreases the number of mast cells in the skin. Clin Exp Allergy 29:1490-1496.

68. Shim, J.Y., B.S. Kim, S.H. Cho, K.U. Min, and S.J. Hong. 2003. Allergen-specific conventional immunotherapy decreases immunoglobulin E-mediated basophil histamine releasability. Clin Exp Allergy 33:52-57.

69. Durham, S.R., S. Ying, V.A. Varney, M.R. Jacobson, R.M. Sudderick, I.S. Mackay, A.B. Kay, and Q.A. Hamid. 1996. Grass pollen immunotherapy inhibits allergen-induced infiltration of CD4+ T lymphocytes and eosinophils in the nasal mucosa and increases the number of cells expressing messenger RNA for interferon-gamma. J Allergy Clin Immunol 97:1356-1365.

70. Ebner, C., S. Schenk, N. Najafian, U. Siemann, R. Steiner, G.W. Fischer, K. Hoffmann, Z. Szepfalusi, O. Scheiner, and D. Kraft. 1995. Nonallergic individuals recognize the same $\mathrm{T}$ cell epitopes of Bet $v 1$, the major birch pollen allergen, as atopic patients. J Immunol 154:1932-1940. 
71. Mark, P.G., D.B. Segal, M.L. Dallaire, and R.D. Garman. 1996. Human T and B cell immune responses to Fel d 1 in cat-allergic and non-cat-allergic subjects. Clin Exp Allergy 26:1316-1328.

72. Carballido, J.M., N. Carballido-Perrig, G. Terres, C.H. Heusser, and K. Blaser. 1992. Bee venom phospholipase A2-specific T cell clones from human allergic and non-allergic individuals: cytokine patterns change in response to the antigen concentration. Eur J Immunol 22:1357-1363.

73. Akdis, M., J. Verhagen, A. Taylor, F. Karamloo, C. Karagiannidis, R. Crameri, S. Thunberg, G. Deniz, R. Valenta, H. Fiebig, C. Kegel, R. Disch, C.B. SchmidtWeber, K. Blaser, and C.A. Akdis. 2004. Immune responses in healthy and allergic individuals are characterized by a fine balance between allergen-specific T regulatory 1 and T helper 2 cells. J Exp Med 199:1567-1575.

74. Akdis, C.A., T. Blesken, M. Akdis, B. Wuthrich, and K. Blaser. 1998. Role of interleukin 10 in specific immunotherapy. J Clin Invest 102:98-106.

75. Ling, E.M., T. Smith, X.D. Nguyen, C. Pridgeon, M. Dallman, J. Arbery, V.A. Carr, and D.S. Robinson. 2004. Relation of CD4+CD25+ regulatory T-cell suppression of allergen-driven T-cell activation to atopic status and expression of allergic disease. Lancet 363:608-615.

76. Verhoef, A., C. Alexander, A.B. Kay, and M. Larche. 2005. T cell epitope immunotherapy induces a CD4+ T cell population with regulatory activity. PLoS Med 2:e78.

77. Akdis, C.A., M. Akdis, T. Blesken, D. Wymann, S.S. Alkan, U. Muller, and K. Blaser. 1996. Epitope-specific T cell tolerance to phospholipase A2 in bee venom immunotherapy and recovery by IL-2 and IL-15 in vitro. J Clin Invest 98:16761683. 
78. Jutel, M., M. Akdis, F. Budak, C. Aebischer-Casaulta, M. Wrzyszcz, K.

Blaser, and C.A. Akdis. 2003. IL-10 and TGF-beta cooperate in the regulatory T cell response to mucosal allergens in normal immunity and specific immunotherapy. Eur J Immunol 33:1205-1214.

79. Francis, J.N., S.J. Till, and S.R. Durham. 2003. Induction of IL-10+CD4+CD25+ T cells by grass pollen immunotherapy. J Allergy Clin Immunol 111:1255-1261.

80. Niederberger, V., F. Horak, S. Vrtala, S. Spitzauer, M.T. Krauth, P. Valent, J. Reisinger, M. Pelzmann, B. Hayek, M. Kronqvist, G. Gafvelin, H. Gronlund, A. Purohit, R. Suck, H. Fiebig, O. Cromwell, G. Pauli, M. van Hage-Hamsten, and R. Valenta. 2004. Vaccination with genetically engineered allergens prevents progression of allergic disease. Proc Natl Acad Sci U S A 101 Suppl 2:1467714682.

81. Karamloo, F., P. Schmid-Grendelmeier, F. Kussebi, M. Akdis, M. Salagianni, B.R. von Beust, A. Reimers, J. Zumkehr, L. Soldatova, Z. Housley-Markovic, U. Muller, T. Kundig, D.M. Kemeny, M.D. Spangfort, K. Blaser, and C.A. Akdis. 2005. Prevention of allergy by a recombinant multi-allergen vaccine with reduced IgE binding and preserved T cell epitopes. Eur J Immunol 35:3268-3276.

82. Kussebi, F., F. Karamloo, C. Rhyner, P. Schmid-Grendelmeier, M. Salagianni, C. Mannhart, M. Akdis, L. Soldatova, Z. Markovic-Housley, B.R. Von Beust, T. Kundig, D.M. Kemeny, K. Blaser, R. Crameri, and C.A. Akdis. 2005. A major allergen gene-fusion protein for potential usage in allergen-specific immunotherapy. J Allergy Clin Immunol 115:323-329.

83. Pree, I., J. Reisinger, M. Focke, S. Vrtala, G. Pauli, M. van Hage, O. Cromwell, E. Gadermaier, C. Egger, N. Reider, F. Horak, R. Valenta, and V. Niederberger. 2007. Analysis of epitope-specific immune responses induced by vaccination 
with structurally folded and unfolded recombinant Bet $v 1$ allergen derivatives in man. J Immunol 179:5309-5316.

84. Tarzi, M., S. Klunker, C. Texier, A. Verhoef, S.O. Stapel, C.A. Akdis, B. Maillere, A.B. Kay, and M. Larche. 2006. Induction of interleukin-10 and suppressor of cytokine signalling-3 gene expression following peptide immunotherapy. Clin Exp Allergy 36:465-474.

85. Oldfield, W.L., M. Larche, and A.B. Kay. 2002. Effect of T-cell peptides derived from Fel d 1 on allergic reactions and cytokine production in patients sensitive to cats: a randomised controlled trial. Lancet 360:47-53.

86. Larche, M. 2007. Update on the current status of peptide immunotherapy. J Allergy Clin Immunol 119:906-909.

87. Counsell, C.M., J.F. Bond, J.L. Ohman, Jr., J.L. Greenstein, and R.D. Garman. 1996. Definition of the human T-cell epitopes of Fel d 1, the major allergen of the domestic cat. J Allergy Clin Immunol 98:884-894.

88. Norman, P.S., J.L. Ohman, Jr., A.A. Long, P.S. Creticos, M.A. Gefter, Z. Shaked, R.A. Wood, P.A. Eggleston, K.B. Hafner, P. Rao, L.M. Lichtenstein, N.H. Jones, and C.F. Nicodemus. 1996. Treatment of cat allergy with T-cell reactive peptides. Am J Respir Crit Care Med 154:1623-1628.

89. Haselden, B.M., A.B. Kay, and M. Larche. 1999. Immunoglobulin E-independent major histocompatibility complex-restricted T cell peptide epitope-induced late asthmatic reactions. J Exp Med 189:1885-1894.

90. Pene, J., A. Desroches, L. Paradis, B. Lebel, M. Farce, C.F. Nicodemus, H. Yssel, and J. Bousquet. 1998. Immunotherapy with Fel d 1 peptides decreases IL-4 release by peripheral blood T cells of patients allergic to cats. J Allergy Clin Immunol 102:571-578. 
91. Marcotte, G.V., C.M. Braun, P.S. Norman, C.F. Nicodemus, A. KageySobotka, L.M. Lichtenstein, and D.M. Essayan. 1998. Effects of peptide therapy on ex vivo T-cell responses. J Allergy Clin Immunol 101:506-513.

92. Oldfield, W.L., A.B. Kay, and M. Larche. 2001. Allergen-derived T cell peptideinduced late asthmatic reactions precede the induction of antigen-specific hyporesponsiveness in atopic allergic asthmatic subjects. J Immunol 167:17341739.

93. Niederberger, V., and R. Valenta. 2004. Recombinant allergens for immunotherapy. Where do we stand? Curr Opin Allergy Clin Immunol 4:549-554.

94. Lutz, M.B., and G. Schuler. 2002. Immature, semi-mature and fully mature dendritic cells: which signals induce tolerance or immunity? Trends Immunol 23:445-449.

95. Steinman, R.M., D. Hawiger, and M.C. Nussenzweig. 2003. Tolerogenic dendritic cells. Annu Rev Immunol 21:685-711.

96. Rutella, S., S. Danese, and G. Leone. 2006. Tolerogenic dendritic cells: cytokine modulation comes of age. Blood 108:1435-1440.

97. van Vugt, M.J., M.J. Kleijmeer, T. Keler, I. Zeelenberg, M.A. van Dijk, J.H. Leusen, H.J. Geuze, and J.G. van de Winkel. 1999. The FcgammaRla (CD64) ligand binding chain triggers major histocompatibility complex class II antigen presentation independently of its associated FcR gamma-chain. Blood 94:808817.

98. Gosselin, E.J., K. Wardwell, D.R. Gosselin, N. Alter, J.L. Fisher, and P.M. Guyre. 1992. Enhanced antigen presentation using human Fc gamma receptor (monocyte/macrophage)-specific immunogens. J Immunol 149:3477-3481. 
99. Keler, T., P.M. Guyre, L.A. Vitale, K. Sundarapandiyan, J.G. van De Winkel, Y.M. Deo, and R.F. Graziano. 2000. Targeting weak antigens to CD64 elicits potent humoral responses in human CD64 transgenic mice. J Immunol 165:6738-6742.

100. Heijnen, I.A., M.J. van Vugt, N.A. Fanger, R.F. Graziano, T.P. de Wit, F.M. Hofhuis, P.M. Guyre, P.J. Capel, J.S. Verbeek, and J.G. van de Winkel. 1996. Antigen targeting to myeloid-specific human Fc gamma RI/CD64 triggers enhanced antibody responses in transgenic mice. J Clin Invest 97:331-338.

101. Guyre, C.A., M.E. Barreda, S.L. Swink, and M.W. Fanger. 2001. Colocalization of Fc gamma RI-targeted antigen with class I MHC: implications for antigen processing. J Immunol 166:2469-2478.

102. Liu, C., J. Goldstein, R.F. Graziano, J. He, J.K. O'Shea, Y. Deo, and P.M. Guyre. 1996. $F(c)$ gammaRl-targeted fusion proteins result in efficient presentation by human monocytes of antigenic and antagonist T cell epitopes. J Clin Invest 98:2001-2007.

103. Wallace, P.K., K.Y. Tsang, J. Goldstein, P. Correale, T.M. Jarry, J. Schlom, P.M. Guyre, M.S. Ernstoff, and M.W. Fanger. 2001. Exogenous antigen targeted to FcgammaRI on myeloid cells is presented in association with MHC class I. J Immunol Methods 248:183-194.

104. Vailes, L.D., A.W. Sun, K. Ichikawa, Z. Wu, T.H. Sulahian, M.D. Chapman, and P.M. Guyre. 2002. High-level expression of immunoreactive recombinant cat allergen (Fel d 1): Targeting to antigen-presenting cells. J Allergy Clin Immunol 110:757-762. 
105. Guyre, C.A., T. Keler, S.L. Swink, L.A. Vitale, R.F. Graziano, and M.W.

Fanger. 2001. Receptor modulation by Fc gamma RI-specific fusion proteins is dependent on receptor number and modified by IgG. J Immunol 167:6303-6311.

106. Hulse, K.E., A.J. Reefer, V.H. Engelhard, S.M. Satinover, J.T. Patrie, M.D. Chapman, and J.A. Woodfolk. 2008. Targeting Fel d 1 to FcgammaRI induces a novel variation of the $\mathrm{T}(\mathrm{H}) 2$ response in subjects with cat allergy. J Allergy Clin Immunol 121:756-762 e754.

107. Friend, S.L., S. Hosier, A. Nelson, D. Foxworthe, D.E. Williams, and A. Farr. 1994. A thymic stromal cell line supports in vitro development of surface IgM+ B cells and produces a novel growth factor affecting $B$ and $T$ lineage cells. Exp Hematol 22:321-328.

108. Sims, J.E., D.E. Williams, P.J. Morrissey, K. Garka, D. Foxworthe, V. Price, S.L. Friend, A. Farr, M.A. Bedell, N.A. Jenkins, N.G. Copeland, K. Grabstein, and R.J. Paxton. 2000. Molecular cloning and biological characterization of a novel murine lymphoid growth factor. J Exp Med 192:671-680.

109. Reche, P.A., V. Soumelis, D.M. Gorman, T. Clifford, M. Liu, M. Travis, S.M. Zurawski, J. Johnston, Y.J. Liu, H. Spits, R. de Waal Malefyt, R.A. Kastelein, and J.F. Bazan. 2001. Human thymic stromal lymphopoietin preferentially stimulates myeloid cells. J Immunol 167:336-343.

110. Quentmeier, H., H.G. Drexler, D. Fleckenstein, M. Zaborski, A. Armstrong, J.E. Sims, and S.D. Lyman. 2001. Cloning of human thymic stromal lymphopoietin (TSLP) and signaling mechanisms leading to proliferation. Leukemia 15:12861292. 
111. Pandey, A., K. Ozaki, H. Baumann, S.D. Levin, A. Puel, A.G. Farr, S.F. Ziegler, W.J. Leonard, and H.F. Lodish. 2000. Cloning of a receptor subunit required for signaling by thymic stromal lymphopoietin. Nat Immunol 1:59-64.

112. Park, L.S., U. Martin, K. Garka, B. Gliniak, J.P. Di Santo, W. Muller, D.A. Largaespada, N.G. Copeland, N.A. Jenkins, A.G. Farr, S.F. Ziegler, P.J. Morrissey, R. Paxton, and J.E. Sims. 2000. Cloning of the murine thymic stromal lymphopoietin (TSLP) receptor: Formation of a functional heteromeric complex requires interleukin 7 receptor. J Exp Med 192:659-670.

113. Liu, Y.J., V. Soumelis, N. Watanabe, T. Ito, Y.H. Wang, W. Malefyt Rde, M. Omori, B. Zhou, and S.F. Ziegler. 2007. TSLP: an epithelial cell cytokine that regulates $\mathrm{T}$ cell differentiation by conditioning dendritic cell maturation. Annu Rev Immunol 25:193-219.

114. Al-Shami, A., R. Spolski, J. Kelly, A. Keane-Myers, and W.J. Leonard. 2005. A role for TSLP in the development of inflammation in an asthma model. $J$ Exp Med 202:829-839.

115. Yoo, J., M. Omori, D. Gyarmati, B. Zhou, T. Aye, A. Brewer, M.R. Comeau, D.J. Campbell, and S.F. Ziegler. 2005. Spontaneous atopic dermatitis in mice expressing an inducible thymic stromal lymphopoietin transgene specifically in the skin. J Exp Med 202:541-549.

116. Zhou, B., M.R. Comeau, T. De Smedt, H.D. Liggitt, M.E. Dahl, D.B. Lewis, D. Gyarmati, T. Aye, D.J. Campbell, and S.F. Ziegler. 2005. Thymic stromal lymphopoietin as a key initiator of allergic airway inflammation in mice. Nat Immunol 6:1047-1053.

117. Ziegler, S.F., and Y.J. Liu. 2006. Thymic stromal lymphopoietin in normal and pathogenic T cell development and function. Nat Immunol 7:709-714. 
118. Soumelis, V., P.A. Reche, H. Kanzler, W. Yuan, G. Edward, B. Homey, M. Gilliet, S. Ho, S. Antonenko, A. Lauerma, K. Smith, D. Gorman, S. Zurawski, J. Abrams, S. Menon, T. McClanahan, R. de Waal-Malefyt Rd, F. Bazan, R.A. Kastelein, and Y.J. Liu. 2002. Human epithelial cells trigger dendritic cell mediated allergic inflammation by producing TSLP. Nat Immunol 3:673-680.

119. Ito, T., Y.H. Wang, O. Duramad, T. Hori, G.J. Delespesse, N. Watanabe, F.X. Qin, Z. Yao, W. Cao, and Y.J. Liu. 2005. TSLP-activated dendritic cells induce an inflammatory T helper type 2 cell response through OX40 ligand. $\mathrm{J}$ Exp Med 202:1213-1223.

120. So, T., J. Song, K. Sugie, A. Altman, and M. Croft. 2006. Signals from OX40 regulate nuclear factor of activated T cells $\mathrm{c} 1$ and T cell helper 2 lineage commitment. Proc Natl Acad Sci U S A 103:3740-3745.

121. Geginat, J., F. Sallusto, and A. Lanzavecchia. 2001. Cytokine-driven proliferation and differentiation of human naive, central memory, and effector memory CD4(+) T cells. J Exp Med 194:1711-1719.

122. Sallusto, F., J. Geginat, and A. Lanzavecchia. 2004. Central memory and effector memory T cell subsets: function, generation, and maintenance. Annu Rev Immunol 22:745-763.

123. Sallusto, F., D. Lenig, R. Forster, M. Lipp, and A. Lanzavecchia. 1999. Two subsets of memory $\mathrm{T}$ lymphocytes with distinct homing potentials and effector functions. Nature 401:708-712.

124. Wang, Y.H., T. Ito, Y.H. Wang, B. Homey, N. Watanabe, R. Martin, C.J. Barnes, B.W. McIntyre, M. Gilliet, R. Kumar, Z. Yao, and Y.J. Liu. 2006. Maintenance and polarization of human $\mathrm{TH} 2$ central memory $\mathrm{T}$ cells by thymic stromal lymphopoietin-activated dendritic cells. Immunity 24:827-838. 
125. Campbell, J.J., K.E. Murphy, E.J. Kunkel, C.E. Brightling, D. Soler, Z. Shen, J. Boisvert, H.B. Greenberg, M.A. Vierra, S.B. Goodman, M.C. Genovese, A.J. Wardlaw, E.C. Butcher, and L. Wu. 2001. CCR7 expression and memory T cell diversity in humans. J Immunol 166:877-884.

126. Rivino, L., M. Messi, D. Jarrossay, A. Lanzavecchia, F. Sallusto, and J. Geginat. 2004. Chemokine receptor expression identifies Pre-T helper (Th)1, Pre-Th2, and nonpolarized cells among human CD4+ central memory T cells. J Exp Med 200:725-735.

127. Wang, Y.H., P. Angkasekwinai, N. Lu, K.S. Voo, K. Arima, S. Hanabuchi, A. Hippe, C.J. Corrigan, C. Dong, B. Homey, Z. Yao, S. Ying, D.P. Huston, and Y.J. Liu. 2007. IL-25 augments type 2 immune responses by enhancing the expansion and functions of TSLP-DC-activated Th2 memory cells. J Exp Med 204:1837-1847.

128. Meissner, N., S. Kochs, J. Coutelle, F. Kussebi, C. Baumgarten, H. Lowenstein, G. Kunkel, and H. Renz. 1999. Modified T-cell activation pattern during specific immunotherapy (SIT) in cat-allergic patients. Clin Exp Allergy 29:618-625.

129. Tighe, H., K. Takabayashi, D. Schwartz, G. Van Nest, S. Tuck, J.J. Eiden, A. Kagey-Sobotka, P.S. Creticos, L.M. Lichtenstein, H.L. Spiegelberg, and E. Raz. 2000. Conjugation of immunostimulatory DNA to the short ragweed allergen amb a 1 enhances its immunogenicity and reduces its allergenicity. J Allergy Clin Immunol 106:124-134.

130. Hochreiter, R., F. Ferreira, J. Thalhamer, and P. Hammerl. 2003. TH1-promoting DNA immunization against allergens modulates the ratio of $\lg \mathrm{G} 1 / \lg \mathrm{g} 2 \mathrm{a}$ but does not affect the anaphylactic potential of IgG1 antibodies: no evidence for the synthesis of nonanaphylactic IgG1. J Allergy Clin Immunol 112:579-584. 
131. Guyre, P.M., R.F. Graziano, J. Goldstein, P.K. Wallace, P.M. Morganelli, K. Wardwell, and A.L. Howell. 1997. Increased potency of Fc-receptor-targeted antigens. Cancer Immunol Immunother 45:146-148.

132. Erwin, E.A., N.J. Custis, S.M. Satinover, M.S. Perzanowski, J.A. Woodfolk, J. Crane, K. Wickens, and T.A. Platts-Mills. 2005. Quantitative measurement of lgE antibodies to purified allergens using streptavidin linked to a high-capacity solid phase. J Allergy Clin Immunol 115:1029-1035.

133. Rowntree, S., T.A. Platts-Mills, J.J. Cogswell, and E.B. Mitchell. 1987. A subclass IgG4-specific antigen-binding radioimmunoassay (RIA): comparison between $\lg G$ and IgG4 antibodies to food and inhaled antigens in adult atopic dermatitis after desensitization treatment and during development of antibody responses in children. J Allergy Clin Immunol 80:622-630.

134. Sallusto, F., and A. Lanzavecchia. 1994. Efficient presentation of soluble antigen by cultured human dendritic cells is maintained by granulocyte/macrophage colony-stimulating factor plus interleukin 4 and downregulated by tumor necrosis factor alpha. J Exp Med 179:1109-1118.

135. Baumgarth, N., and M. Roederer. 2000. A practical approach to multicolor flow cytometry for immunophenotyping. J Immunol Methods 243:77-97.

136. Akdis, M. 2006. Healthy immune response to allergens: T regulatory cells and more. Curr Opin Immunol 18:738-744.

137. van Duivenvoorde, L.M., G.J. van Mierlo, Z.F. Boonman, and R.E. Toes. 2006. Dendritic cells: vehicles for tolerance induction and prevention of autoimmune diseases. Immunobiology 211:627-632. 
138. Zhu, D., C.L. Kepley, K. Zhang, T. Terada, T. Yamada, and A. Saxon. 2005. A chimeric human-cat fusion protein blocks cat-induced allergy. Nat Med 11:446449.

139. Macaubas, C., J. Wahlstrom, A.P. Galvao da Silva, T.G. Forsthuber, G. Sonderstrup, W.W. Kwok, R.H. DeKruyff, and D.T. Umetsu. 2006. Allergenspecific MHC class II tetramer+ cells are detectable in allergic, but not in nonallergic, individuals. J Immunol 176:5069-5077.

140. Akbari, O., R.H. DeKruyff, and D.T. Umetsu. 2001. Pulmonary dendritic cells producing IL-10 mediate tolerance induced by respiratory exposure to antigen. Nat Immunol 2:725-731.

141. Stock, P., T. Kallinich, O. Akbari, D. Quarcoo, K. Gerhold, U. Wahn, D.T. Umetsu, and E. Hamelmann. 2004. CD8(+) T cells regulate immune responses in a murine model of allergen-induced sensitization and airway inflammation. Eur J Immunol 34:1817-1827.

142. Woodfolk, J.A., and T.A. Platts-Mills. 2001. Diversity of the human allergenspecific T cell repertoire associated with distinct skin test reactions: delayed-type hypersensitivity-associated major epitopes induce Th1- and Th2-dominated responses. J Immunol 167:5412-5419.

143. Liu, Y.J. 2007. Thymic stromal lymphopoietin and OX40 ligand pathway in the initiation of dendritic cell-mediated allergic inflammation. J Allergy Clin Immunol 120:238-244; quiz 245-236.

144. Ying, S., B. O'Connor, J. Ratoff, Q. Meng, K. Mallett, D. Cousins, D. Robinson, G. Zhang, J. Zhao, T.H. Lee, and C. Corrigan. 2005. Thymic stromal lymphopoietin expression is increased in asthmatic airways and correlates with 
expression of Th2-attracting chemokines and disease severity. J Immunol 174:8183-8190.

145. Kato, A., S. Favoreto, Jr., P.C. Avila, and R.P. Schleimer. 2007. TLR3- and Th2 cytokine-dependent production of thymic stromal lymphopoietin in human airway epithelial cells. J Immunol 179:1080-1087.

146. Bochner, B.S., S.A. Hudson, H.Q. Xiao, and M.C. Liu. 2003. Release of both CCR4-active and CXCR3-active chemokines during human allergic pulmonary late-phase reactions. J Allergy Clin Immunol 112:930-934.

147. Uchida, T., H. Suto, C. Ra, H. Ogawa, T. Kobata, and K. Okumura. 2002. Preferential expression of $\mathrm{T}(\mathrm{h}) 2$-type chemokine and its receptor in atopic dermatitis. Int Immunol 14:1431-1438.

148. Vestergaard, C., K. Bang, B. Gesser, H. Yoneyama, K. Matsushima, and C.G. Larsen. 2000. A Th2 chemokine, TARC, produced by keratinocytes may recruit CLA+CCR4+ lymphocytes into lesional atopic dermatitis skin. J Invest Dermatol 115:640-646.

149. Panina-Bordignon, P., A. Papi, M. Mariani, P. Di Lucia, G. Casoni, C. Bellettato, C. Buonsanti, D. Miotto, C. Mapp, A. Villa, G. Arrigoni, L.M. Fabbri, and F. Sinigaglia. 2001. The C-C chemokine receptors CCR4 and CCR8 identify airway T cells of allergen-challenged atopic asthmatics. J Clin Invest 107:1357-1364.

150. Morgan, A.J., F.A. Symon, M.A. Berry, I.D. Pavord, C.J. Corrigan, and A.J. Wardlaw. 2005. IL-4-expressing bronchoalveolar T cells from asthmatic and healthy subjects preferentially express CCR 3 and CCR 4. J Allergy Clin Immunol 116:594-600.

151. Hartl, D., M. Griese, T. Nicolai, G. Zissel, C. Prell, N. Konstantopoulos, R. Gruber, D. Reinhardt, D.J. Schendel, and S. Krauss-Etschmann. 2005. 
Pulmonary chemokines and their receptors differentiate children with asthma and chronic cough. J Allergy Clin Immunol 115:728-736.

152. Nouri-Aria, K.T., D. Wilson, J.N. Francis, L.A. Jopling, M.R. Jacobson, M.R. Hodge, D.P. Andrew, S.J. Till, E.M. Varga, T.J. Williams, J.E. Pease, C.M. Lloyd, I. Sabroe, and S.R. Durham. 2002. CCR4 in human allergen-induced late responses in the skin and lung. Eur J Immunol 32:1933-1938.

153. Campbell, J.J., G. Haraldsen, J. Pan, J. Rottman, S. Qin, P. Ponath, D.P. Andrew, R. Warnke, N. Ruffing, N. Kassam, L. Wu, and E.C. Butcher. 1999. The chemokine receptor CCR4 in vascular recognition by cutaneous but not intestinal memory T cells. Nature 400:776-780.

154. Hijnen, D., M. De Bruin-Weller, B. Oosting, C. Lebre, E. De Jong, C. BruijnzeelKoomen, and E. Knol. 2004. Serum thymus and activation-regulated chemokine (TARC) and cutaneous T cell- attracting chemokine (CTACK) levels in allergic diseases: TARC and CTACK are disease-specific markers for atopic dermatitis. J Allergy Clin Immunol 113:334-340.

155. Kunz, B., A.P. Oranje, L. Labreze, J.F. Stalder, J. Ring, and A. Taieb. 1997. Clinical validation and guidelines for the SCORAD index: consensus report of the European Task Force on Atopic Dermatitis. Dermatology 195:10-19.

156. Roederer, M. 2001. Spectral compensation for flow cytometry: visualization artifacts, limitations, and caveats. Cytometry 45:194-205.

157. Rochman, I., N. Watanabe, K. Arima, Y.J. Liu, and W.J. Leonard. 2007. Cutting edge: direct action of thymic stromal lymphopoietin on activated human CD4+ T cells. J Immunol 178:6720-6724. 
158. Harrison, P.T., W. Davis, J.C. Norman, A.R. Hockaday, and J.M. Allen. 1994.

Binding of monomeric immunoglobulin G triggers Fc gamma RI-mediated endocytosis. J Biol Chem 269:24396-24402.

159. Kiefer, F., J. Brumell, N. Al-Alawi, S. Latour, A. Cheng, A. Veillette, S. Grinstein, and T. Pawson. 1998. The Syk protein tyrosine kinase is essential for Fcgamma receptor signaling in macrophages and neutrophils. Mol Cell Biol 18:4209-4220.

160. Rowley, R.B., A.L. Burkhardt, H.G. Chao, G.R. Matsueda, and J.B. Bolen. 1995. Syk protein-tyrosine kinase is regulated by tyrosine-phosphorylated lg alpha/lg beta immunoreceptor tyrosine activation motif binding and autophosphorylation. $J$ Biol Chem 270:11590-11594.

161. Durden, D.L., H.M. Kim, B. Calore, and Y. Liu. 1995. The Fc gamma RI receptor signals through the activation of hck and MAP kinase. J Immunol 154:4039-4047.

162. Eisenbarth, S.C., D.A. Piggott, J.W. Huleatt, I. Visintin, C.A. Herrick, and K. Bottomly. 2002. Lipopolysaccharide-enhanced, toll-like receptor 4-dependent T helper cell type 2 responses to inhaled antigen. J Exp Med 196:1645-1651.

163. Ng, N., D. Lam, P. Paulus, G. Batzer, and A.A. Horner. 2006. House dust extracts have both $\mathrm{TH} 2$ adjuvant and tolerogenic activities. J Allergy Clin Immunol 117:1074-1081.

164. Kim, Y.K., S.Y. Oh, S.G. Jeon, H.W. Park, S.Y. Lee, E.Y. Chun, B. Bang, H.S. Lee, M.H. Oh, Y.S. Kim, J.H. Kim, Y.S. Gho, S.H. Cho, K.U. Min, Y.Y. Kim, and Z. Zhu. 2007. Airway exposure levels of lipopolysaccharide determine type 1 versus type 2 experimental asthma. J Immunol 178:5375-5382.

165. Lam, D., N. Ng, S. Lee, G. Batzer, and A.A. Horner. 2008. Airway house dust extract exposures modify allergen-induced airway hypersensitivity responses by TLR4-dependent and independent pathways. J Immunol 181:2925-2932. 
166. Guyre, P.M., P.M. Morganelli, and R. Miller. 1983. Recombinant immune interferon increases immunoglobulin G Fc receptors on cultured human mononuclear phagocytes. J Clin Invest 72:393-397.

167. te Velde, A.A., R. de Waal Malefijt, R.J. Huijbens, J.E. de Vries, and C.G. Figdor. 1992. IL-10 stimulates monocyte Fc gamma R surface expression and cytotoxic activity. Distinct regulation of antibody-dependent cellular cytotoxicity by IFNgamma, IL-4, and IL-10. J Immunol 149:4048-4052.

168. Gessl, A., M. Willheim, A. Spittler, H. Agis, W. Krugluger, and G. Boltz-Nitulescu. 1994. Influence of tumour necrosis factor-alpha on the expression of Fc IgG and IgA receptors, and other markers by cultured human blood monocytes and U937 cells. Scand J Immunol 39:151-156.

169. Huang, Z.Y., D.R. Barreda, R.G. Worth, Z.K. Indik, M.K. Kim, P. Chien, and A.D. Schreiber. 2006. Differential kinase requirements in human and mouse Fcgamma receptor phagocytosis and endocytosis. J Leukoc Biol 80:1553-1562.

170. Norman, J.C., and J.M. Allen. 2000. Endocytosis of FcgammaRI is regulated by two distinct signalling pathways. FEBS Lett 484:179-183.

171. Novak, N., C. Tepel, S. Koch, K. Brix, T. Bieber, and S. Kraft. 2003. Evidence for a differential expression of the FcepsilonRIgamma chain in dendritic cells of atopic and nonatopic donors. J Clin Invest 111:1047-1056.

172. Novak, N., T. Bieber, and S. Kraft. 2004. Immunoglobulin E-bearing antigenpresenting cells in atopic dermatitis. Curr Allergy Asthma Rep 4:263-269.

173. Isaksen, D.E., H. Baumann, B. Zhou, S. Nivollet, A.G. Farr, S.D. Levin, and S.F. Ziegler. 2002. Uncoupling of proliferation and Stat5 activation in thymic stromal lymphopoietin-mediated signal transduction. J Immunol 168:3288-3294. 
174. Bernasconi, A., R. Marino, A. Ribas, J. Rossi, M. Ciaccio, M. Oleastro, A. Ornani, R. Paz, M.A. Rivarola, M. Zelazko, and A. Belgorosky. 2006.

Characterization of immunodeficiency in a patient with growth hormone insensitivity secondary to a novel STAT5b gene mutation. Pediatrics 118:e15841592.

175. Kim, H.P., J. Imbert, and W.J. Leonard. 2006. Both integrated and differential regulation of components of the IL-2/IL-2 receptor system. Cytokine Growth Factor Rev 17:349-366.

176. Markiewicz, S., R. Bosselut, F. Le Deist, J.P. de Villartay, C. Hivroz, J. Ghysdael, A. Fischer, and G. de Saint Basile. 1996. Tissue-specific activity of the gammac chain gene promoter depends upon an Ets binding site and is regulated by GAbinding protein. J Biol Chem 271:14849-14855.

177. Steer, J.H., K.M. Kroeger, L.J. Abraham, and D.A. Joyce. 2000. Glucocorticoids suppress tumor necrosis factor-alpha expression by human monocytic THP-1 cells by suppressing transactivation through adjacent NF-kappa B and c-Junactivating transcription factor-2 binding sites in the promoter. $\mathrm{J}$ Biol Chem 275:18432-18440.

178. Hoffmeyer, A., A. Avots, E. Flory, C.K. Weber, E. Serfling, and U.R. Rapp. 1998. The GABP-responsive element of the interleukin-2 enhancer is regulated by JNK/SAPK-activating pathways in T lymphocytes. J Biol Chem 273:1011210119.

179. Tsokos, G.C., M.P. Nambiar, and Y.T. Juang. 2003. Activation of the Ets transcription factor Elf-1 requires phosphorylation and glycosylation: defective expression of activated Elf-1 is involved in the decreased TCR zeta chain gene 
expression in patients with systemic lupus erythematosus. Ann N Y Acad Sci 987:240-245.

180. Besin, G., S. Gaudreau, M. Menard, C. Guindi, G. Dupuis, and A. Amrani. 2008. Thymic stromal lymphopoietin and thymic stromal lymphopoietin-conditioned dendritic cells induce regulatory T-cell differentiation and protection of NOD mice against diabetes. Diabetes 57:2107-2117. 